\title{
On the Limits of HOMO Driven Reactions: The Frontier Effective-for-Reaction Molecular Orbital Concept.
}

Rodrigo R. da Silva, Teodorico C. Ramalho, Joana M. Santos and J. D. Figueroa-Villar.

\section{Supporting Information}

Table S1. List of Compounds.

\begin{tabular}{cccc}
\hline \multicolumn{4}{c}{ COMPOUNDS } \\
\hline 1 & 2,2 -dimethyl-propionic acid & 28 & 2-chloro-propionic acid \\
2 & propionic acid & 29 & fluoro-acetic acid \\
3 & butyric acid & 30 & cyano-acetic acid \\
4 & acetic acid & 31 & nitro-acetic acid \\
5 & $p$-methyl-benzoic acid & 32 & dichloro-acetic acid \\
6 & vinyl-acetic acid & 33 & oxalic acid \\
7 & phenyl-acetic acid & 34 & difluoro-acetic acid \\
8 & $m$-methyl-benzoic acid & 35 & trichloro-acetic acid \\
9 & succinic acid & 36 & trifluoro-acetic acid \\
10 & benzoic acid & 37 & $t$-butyl alcohol \\
11 & $p$-fluoro-benzoic acid & 38 & iso-propanol \\
12 & 3 -chloro-propionic acid & 39 & $n$-propanol \\
13 & $p$-chloro-benzoic acid & 40 & ethanol \\
14 & $p$-bromo-benzoic acid & 41 & methanol \\
15 & $m$-fluoro-benzoic acid & 42 & $2,2,2$-trifluoro-ethanol \\
16 & $m$-chloro-benzoic acid & 43 & $p$-amino-phenol \\
17 & glycolic acid & 44 & $p$-methoxy-phenol \\
18 & $m$-bromo-benzoic acid & 45 & $p$-methyl-phenol \\
19 & formic acid & 46 & $m$-methyl-phenol \\
20 & $m$-cyano-benzoic acid & 47 & phenol \\
21 & $p$-cyano-benzoic acid & 48 & $p$-hydroxy-phenol \\
22 & methoxy-acetic acid & 49 & $p$-fluoro-phenol \\
23 & 3 -butynoic acid & 50 & $m$-amino-phenol \\
24 & fumaric acid & 51 & $m$-methoxy-phenol \\
25 & bromo-acetic acid & 52 & $m$-hydroxy-phenol \\
26 & malonic acid & 53 & $p$-chloro-phenol \\
27 & chloro-acetic acid & 54 & $p$-bromo-phenol
\end{tabular}




\begin{tabular}{llll}
55 & $m$-fluoro-phenol & 59 & $m$-nitro-phenol \\
56 & $m$-bromo-phenol & 60 & $p$-cyano-phenol \\
57 & $m$-chloro-phenol & 61 & $p$-nitro-phenol \\
58 & $m$-cyano-phenol & 62 & furfuryl amine \\
\hline
\end{tabular}

Part S1. Cartesian coordinates for stationary points

\section{Compound 1:}

$\begin{array}{lrrr}\text { C1 } & 0.000000 & 0.000000 & 0.000000 \\ \text { O2 } & 0.000000 & 0.000000 & 1.255122 \\ \text { O3 } & 0.971821 & 0.000000 & -0.800094 \\ \text { C4 } & -1.431455 & 0.000224 & -0.710161 \\ \text { C5 } & -2.565467 & -0.000812 & 0.320877 \\ \text { H6 } & -2.491463 & 0.875327 & 0.972146 \\ \text { H7 } & -3.556628 & -0.000334 & -0.161571 \\ \text { H8 } & -2.491544 & -0.878288 & 0.970375 \\ \text { C9 } & -1.515223 & 1.252444 & -1.599706 \\ \text { H10 } & -1.504817 & 2.166010 & -0.990824 \\ \text { H11 } & -0.642251 & 1.279880 & -2.257688 \\ \text { H12 } & -2.431595 & 1.269613 & -2.211518 \\ \text { C13 } & -1.514812 & -1.250467 & -1.601862 \\ \text { H14 } & -0.641720 & -1.276541 & -2.259736 \\ \text { H15 } & -1.504275 & -2.165102 & -0.994585 \\ \text { H16 } & -2.431106 & -1.266796 & -2.213815\end{array}$

\section{Compound 3:}

$\begin{array}{lrrr}\text { C1 } & 0.000000 & 0.000000 & 0.000000 \\ \text { O2 } & 0.000000 & 0.000000 & 1.261122 \\ \text { O3 } & 0.967969 & 0.000000 & -0.797384 \\ \text { C4 } & -1.418507 & -0.028017 & -0.692906 \\ \text { H5 } & -1.423869 & 0.730082 & -1.490143 \\ \text { H6 } & -1.506114 & -0.995993 & -1.209233 \\ \text { C7 } & -2.609293 & 0.168534 & 0.251197 \\ \text { H8 } & -3.558539 & -0.057930 & -0.263441 \\ \text { H9 } & -2.500244 & -0.545180 & 1.075886 \\ \text { C10 } & -2.665306 & 1.580091 & 0.844424 \\ \text { H11 } & -3.506087 & 1.704063 & 1.541412 \\ \text { H12 } & -1.729887 & 1.758095 & 1.380576 \\ \text { H13 } & -2.771836 & 2.340036 & 0.057561 \\ \text { Compound 6: } & & \\ \text { C1 } & 0.000000 & 0.000000 & 0.000000 \\ \text { O2 } & 0.000000 & 0.000000 & 1.250472 \\ \text { O3 } & 0.950213 & 0.000000 & -0.820288 \\ \text { C4 } & -1.450367 & 0.068746 & -0.668768 \\ \text { H5 } & -1.584600 & 1.130388 & -0.934256 \\ \text { H6 } & -2.222539 & -0.187721 & 0.065375 \\ \text { C7 } & -1.555056 & -0.756528 & -1.905331 \\ \text { C8 } & -2.531673 & -1.614830 & -2.225090 \\ \text { H9 } & -0.692434 & -0.647176 & -2.563045 \\ \text { H10 } & -2.498892 & -2.211544 & -3.135131 \\ \text { H11 } & -3.395085 & -1.767157 & -1.578560\end{array}$

\section{Compound 2:}

$\begin{array}{rrrr}\text { C1 } & 0.000000 & 0.000000 & 0.000000 \\ \text { O2 } & 0.000000 & 0.000000 & 1.258295 \\ \text { O3 } & 0.968525 & 0.000000 & -0.798922 \\ \text { C4 } & -1.415710 & 0.000788 & -0.712744 \\ \text { H5 } & -1.430198 & 0.871357 & -1.382043 \\ \text { H6 } & -1.431619 & -0.870529 & -1.381045 \\ \text { C7 } & -2.623569 & 0.002249 & 0.239587 \\ \text { H8 } & -3.265293 & 0.886039 & 0.117866 \\ \text { H9 } & -3.269079 & -0.878520 & 0.115942 \\ \text { H10 } & -2.218821 & 0.000372 & 1.258161\end{array}$

Compound 4:

$\begin{array}{rrrr}\text { C1 } & 0.220146 & 0.001748 & -0.000085 \\ \text { O2 } & 0.807242 & -1.109166 & 0.000032 \\ \text { O3 } & 0.697051 & 1.165487 & 0.000010 \\ \text { C4 } & -1.354158 & -0.054185 & -0.000006 \\ \text { H5 } & -1.748411 & 0.471136 & 0.880944 \\ \text { H6 } & -1.748646 & 0.475983 & -0.877941 \\ \text { H7 } & -1.733220 & -1.083069 & -0.002793\end{array}$

Compond 5:

$\begin{array}{llll}\text { C1 } & 0.000000 & 0.000000 & 0.000000\end{array}$

$\begin{array}{lllll}\mathrm{O} 2 & 0.000000 & 0.000000 & 1.256636\end{array}$

$\begin{array}{lllll}03 & 0.962478 & 0.000000 & -0.808273\end{array}$

$\begin{array}{lllll}\text { C4 } & -1.409254 & 0.000000 & -0.657230\end{array}$

$\begin{array}{llll}\text { C5 } & -1.554335 & 0.000089 & -2.050181\end{array}$

$\begin{array}{lllll}\text { C6 } & -2.815941 & 0.000041 & -2.643244\end{array}$

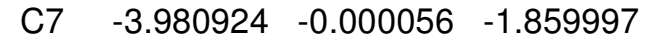

C8 $\quad-3.834421-0.000205-0.466760$

$\begin{array}{llll}\text { C9 } & -2.568400 & -0.000158 & 0.123415\end{array}$

$\begin{array}{llll}\mathrm{H} 10 & -0.639950 & 0.000121 & -2.636848\end{array}$

$\begin{array}{llll}\mathrm{H} 11 & -2.906759 & 0.000046 & -3.730295\end{array}$

$\begin{array}{llll}\mathrm{H} 12 & -4.727518 & -0.000384 & 0.159113\end{array}$

$\begin{array}{llll}\mathrm{H} 13 & -2.433789 & -0.000315 & 1.201435\end{array}$

$\begin{array}{lllll}\text { C14 } & -5.349777 & 0.000426 & -2.504560\end{array}$

$\begin{array}{lllll}\mathrm{H} 15 & -5.504403 & 0.883830 & -3.138473\end{array}$

$\begin{array}{llll}\mathrm{H} 16 & -5.500918 & -0.877366 & -3.146982\end{array}$

$\begin{array}{llll}H 17 & -6.143730 & -0.004690 & -1.750151\end{array}$ 


\section{Compound 7:}

$\begin{array}{lrrr}\text { C1 } & 0.000000 & 0.000000 & 0.000000 \\ \text { O2 } & 0.000000 & 0.000000 & 1.256123 \\ \text { O3 } & 0.948687 & 0.000000 & -0.811991 \\ \text { C4 } & -1.451226 & -0.079156 & -0.672973 \\ \text { H5 } & -1.341464 & -0.025953 & -1.760562 \\ \text { H6 } & -1.821124 & -1.084849 & -0.425389 \\ \text { C7 } & -2.448780 & 0.938710 & -0.197111 \\ \text { C8 } & -2.736769 & 1.086276 & 1.174329 \\ \text { C9 } & -3.665252 & 2.027386 & 1.613881 \\ \text { C10 } & -4.336651 & 2.851301 & 0.703409 \\ \text { C11 } & -4.054006 & 2.725466 & -0.657262 \\ \text { C12 } & -3.122164 & 1.781651 & -1.096350 \\ \text { H13 } & -2.162682 & 0.477747 & 1.865429 \\ \text { H14 } & -3.866478 & 2.124744 & 2.679487 \\ \text { H15 } & -5.063749 & 3.582395 & 1.050892 \\ \text { H16 } & -4.559872 & 3.362411 & -1.380980 \\ \text { H17 } & -2.909078 & 1.691571 & -2.159886\end{array}$

\section{Compound 9:}

$\begin{array}{rrrr}\text { C1 } & 1.881942 & -0.011377 & -0.140437 \\ \text { O2 } & 2.759664 & -0.651007 & -0.696199 \\ \text { O3 } & 2.152947 & 0.621564 & 1.045497 \\ \text { H4 } & 3.076665 & 0.386563 & 1.231815 \\ \text { C5 } & 0.467133 & 0.177157 & -0.589889 \\ \text { H6 } & 0.182317 & 1.235876 & -0.575265 \\ \text { H7 } & 0.401079 & -0.176444 & -1.622075 \\ \text { C8 } & -0.574705 & -0.532219 & 0.296256 \\ \text { H9 } & -0.551400 & -1.618496 & 0.157851 \\ \text { H10 } & -0.347634 & -0.338520 & 1.352969 \\ \text { C11 } & -2.041535 & 0.001626 & 0.020781 \\ \text { O12 } & -2.108784 & 1.158751 & -0.466541 \\ \text { O13 } & -2.948582 & -0.791821 & 0.359049\end{array}$

\section{Compound 11:}

$\begin{array}{rrrr}\text { C1 } & 0.000000 & 0.000000 & 0.000000 \\ \text { O2 } & 0.000000 & 0.000000 & 1.256232 \\ \text { O3 } & 0.959562 & 0.000000 & -0.810782 \\ \text { C4 } & -1.410827 & -0.000027 & -0.654856 \\ \text { C5 } & -1.553704 & 0.000021 & -2.046970 \\ \text { C6 } & -2.814976 & -0.000007 & -2.647976 \\ \text { C7 } & -3.937234 & -0.000081 & -1.827798 \\ \text { C8 } & -3.839501 & -0.000128 & -0.441264 \\ \text { C9 } & -2.566396 & -0.000102 & 0.134370 \\ \text { H10 } & -0.638556 & 0.000081 & -2.631805 \\ \text { H11 } & -2.943226 & 0.000030 & -3.727324 \\ \text { H12 } & -4.746705 & -0.000187 & 0.157415 \\ \text { H13 } & -2.422652 & -0.000140 & 1.210873 \\ \text { F14 } & -5.178169 & -0.000108 & -2.403960\end{array}$

\section{Compound 13:}

$\begin{array}{lrrr}\text { C1 } & 0.954849 & -1.217464 & 0.000240 \\ \text { C2 } & -0.441366 & -1.201892 & 0.000280 \\ \text { C3 } & -1.156920 & 0.000006 & 0.000032 \\ \text { C4 } & -0.441336 & 1.201912 & -0.000233 \\ \text { C5 } & 0.954884 & 1.217469 & -0.000224 \\ \text { C6 } & 1.633276 & 0.000005 & 0.000002 \\ \text { H7 } & 1.511760 & -2.149958 & 0.000424\end{array}$

\section{Compound 8:}

$\begin{array}{lrrr}\text { C1 } & 0.000000 & 0.000000 & 0.000000 \\ \text { O2 } & 0.000000 & 0.000000 & 1.256613 \\ \text { O3 } & 0.962726 & 0.000000 & -0.807791 \\ \text { C4 } & -1.411083 & -0.000015 & -0.656910 \\ \text { C5 } & -1.549042 & 0.000195 & -2.046931 \\ \text { C6 } & -2.806396 & 0.000178 & -2.666456 \\ \text { C7 } & -3.949916 & -0.000053 & -1.857674 \\ \text { C8 } & -3.830031 & -0.000260 & -0.463371 \\ \text { C9 } & -2.569317 & -0.000246 & 0.131115 \\ \text { H10 } & -0.626917 & 0.000375 & -2.623966 \\ \text { H11 } & -4.936529 & -0.000071 & -2.320310 \\ \text { H12 } & -4.728935 & -0.000445 & 0.152368 \\ \text { H13 } & -2.430895 & -0.000416 & 1.208344 \\ \text { C14 } & -2.922964 & 0.000435 & -4.175904 \\ \text { H15 } & -2.439340 & 0.880479 & -4.618662 \\ \text { H16 } & -2.438795 & -0.879137 & -4.619000 \\ \text { H17 } & -3.970031 & 0.000178 & -4.499373\end{array}$

\section{Compound 10:}

$\begin{array}{cccc}\text { C1 } & 0.000000 & 0.000000 & 0.000000 \\ \text { C2 } & 0.000000 & 0.000000 & 1.396363 \\ \text { C3 } & 1.199984 & 0.000000 & 2.117114 \\ \text { C4 } & 2.405288 & 0.000018 & 1.405170 \\ \text { C5 } & 2.415486 & 0.000033 & 0.008911 \\ \text { C6 } & 1.210292 & 0.000012 & -0.700865 \\ \text { H7 } & -0.941883 & -0.000023 & -0.547803 \\ \text { H8 } & -0.918213 & 0.000015 & 1.977008 \\ \text { H9 } & 3.318989 & -0.000001 & 1.992844 \\ \text { H10 } & 3.361290 & 0.000059 & -0.532085 \\ \text { H11 } & 1.214369 & 0.000012 & -1.789672 \\ \text { C12 } & 1.194282 & -0.000001 & 3.673007 \\ \text { O13 } & 0.053273 & -0.000456 & 4.199522 \\ \text { O14 } & 2.331329 & 0.000053 & 4.208227 \\ \text { Compound 12: } & & \\ \text { C1 } & 0.000000 & 0.000000 & 0.000000 \\ \text { O2 } & 0.000000 & 0.000000 & 1.258916 \\ \text { O3 } & 0.946206 & 0.000000 & -0.813284 \\ \text { C4 } & -1.445290 & 0.000111 & -0.661319 \\ \text { H5 } & -1.540814 & 0.880287 & -1.307259 \\ \text { H6 } & -1.540808 & -0.879833 & -1.307576 \\ \text { C7 } & -2.500070 & -0.000074 & 0.413648 \\ \text { H8 } & -2.456013 & -0.885560 & 1.042707 \\ \text { H9 } & -2.455910 & 0.885134 & 1.043088 \\ \text { Cl10 } & -4.265958 & 0.000157 & -0.273612\end{array}$

$\begin{array}{rrrr}\text { H8 } & -1.023739 & -2.118715 & 0.000533 \\ \text { H9 } & -1.023623 & 2.118795 & -0.000470 \\ \text { H10 } & 1.511813 & 2.149962 & -0.000417 \\ \text { C11 } & -2.712925 & -0.000009 & 0.000049 \\ \text { O12 } & -3.239229 & -1.139968 & -0.000645 \\ \text { O13 } & -3.239163 & 1.139980 & 0.000564 \\ \text { Cl14 } & 3.418127 & -0.000020 & -0.000018\end{array}$


Compound 14:

$\begin{array}{rrrr}\text { C1 } & 0.000000 & 0.000000 & 0.000000 \\ \text { O2 } & 0.000000 & 0.000000 & 1.255517 \\ \text { O3 } & 0.955026 & 0.000000 & -0.815025 \\ \text { C4 } & -1.413070 & -0.000032 & -0.651669 \\ \text { C5 } & -1.558723 & 0.000069 & -2.042975 \\ \text { C6 } & -2.820072 & 0.000037 & -2.642085 \\ \text { C7 } & -3.944116 & -0.000094 & -1.819181 \\ \text { C8 } & -3.840445 & -0.000195 & -0.430016 \\ \text { C9 } & -2.565922 & -0.000165 & 0.140627 \\ \text { H10 } & -0.645732 & 0.000175 & -2.631516 \\ \text { H11 } & -2.934413 & 0.000115 & -3.721890 \\ \text { H12 } & -4.736029 & -0.000299 & 0.183976 \\ \text { H13 } & -2.421073 & -0.000246 & 1.217169 \\ \text { Br14 } & -5.703670 & -0.000138 & -2.630844 \\ \text { Compound 16: } & & \\ \text { C1 } & 0.000000 & 0.000000 & 0.000000 \\ \text { C2 } & 0.000000 & 0.000000 & 1.395146 \\ \text { C3 } & 1.201138 & 0.000000 & 2.111859 \\ \text { C4 } & 2.412901 & 0.000000 & 1.410472 \\ \text { C5 } & 2.394009 & 0.000002 & 0.021446 \\ \text { C6 } & 1.205709 & 0.000002 & -0.708185 \\ \text { H7 } & -0.938291 & 0.000001 & -0.552381 \\ \text { H8 } & -0.915784 & 0.000001 & 1.978212 \\ \text { H9 } & 3.329175 & -0.000001 & 1.990951 \\ \text { C10 } & 1.202547 & -0.000001 & 3.672099 \\ \text { O11 } & 0.061463 & 0.000076 & 4.194436 \\ \text { O12 } & 2.341528 & -0.000057 & 4.197786 \\ \text { H13 } & 1.226473 & 0.000004 & -1.793153 \\ \text { Cl14 } & 3.939215 & 0.000001 & -0.881895 \\ \text { Comp } & 047 d 19: & & \end{array}$

\section{Compound 19:}

$\begin{array}{crrr}\text { C1 } & -0.306213 & -0.055935 & 0.000000 \\ \text { O2 } & 0.409686 & -1.085687 & 0.000000 \\ \text { O3 } & 0.000000 & 1.160347 & 0.000000 \\ \text { H4 } & -1.440215 & -0.261662 & 0.000000 \\ \text { Compound 20: } & & \\ \text { C1 } & 0.000000 & 0.000000 & 0.000000 \\ \text { O2 } & 0.000000 & 0.000000 & 1.254600 \\ \text { O3 } & 0.948429 & 0.000000 & -0.820289 \\ \text { C4 } & -1.418416 & -0.000099 & -0.646363 \\ \text { C5 } & -1.566226 & -0.000076 & -2.034240 \\ \text { C6 } & -2.846411 & -0.000188 & -2.615282 \\ \text { C7 } & -3.994642 & -0.000324 & -1.798659 \\ \text { C8 } & -3.843597 & -0.000342 & -0.414466 \\ \text { C9 } & -2.566256 & -0.000229 & 0.152978 \\ \text { H10 } & -0.660687 & 0.000016 & -2.632565 \\ \text { H11 } & -4.979946 & -0.000410 & -2.255476 \\ \text { H12 } & -4.727466 & -0.000449 & 0.220157 \\ \text { H13 } & -2.406515 & -0.000239 & 1.227383 \\ \text { C14 } & -3.003012 & -0.000161 & -4.041129 \\ \text { N15 } & -3.145712 & -0.000140 & -5.198040\end{array}$

\section{Compound 15:}

$\begin{array}{lrrr}\text { C1 } & 0.000000 & 0.000000 & 0.000000 \\ \text { O2 } & 0.000000 & 0.000000 & 1.255659 \\ \text { O3 } & 0.956680 & 0.000000 & -0.812989 \\ \text { C4 } & -1.414995 & -0.000016 & -0.653053 \\ \text { C5 } & -1.545589 & 0.000097 & -2.046271 \\ \text { C6 } & -2.812919 & 0.000074 & -2.608534 \\ \text { C7 } & -3.973560 & -0.000053 & -1.841756 \\ \text { C8 } & -3.837856 & -0.000162 & -0.448805 \\ \text { C9 } & -2.571711 & -0.000146 & 0.137119 \\ \text { H10 } & -0.641637 & 0.000200 & -2.646331 \\ \text { H11 } & -4.943498 & -0.000066 & -2.330205 \\ \text { H12 } & -4.732249 & -0.000264 & 0.172389 \\ \text { H13 } & -2.428356 & -0.000236 & 1.213135 \\ \text { F14 } & -2.942662 & 0.000183 & -3.971040 \\ \text { Compound 17: } & & \\ \text { C1 } & 0.691850 & 0.076874 & 0.000172 \\ \text { O2 } & 0.684975 & 1.325478 & -0.000029 \\ \text { O3 } & 1.647631 & -0.742941 & -0.000078 \\ \text { C4 } & -0.693469 & -0.658982 & 0.000031 \\ \text { H5 } & -0.714850 & -1.319289 & -0.884357 \\ \text { H6 } & -0.714995 & -1.319341 & 0.884375 \\ \text { O7 } & -1.826817 & 0.227015 & -0.000039 \\ \text { H8 } & -2.606752 & -0.345135 & -0.000064\end{array}$

\section{Compound 18:}

$\begin{array}{rrrr}\text { C1 } & 0.000000 & 0.000000 & 0.000000 \\ \text { O2 } & 0.000000 & 0.000000 & 1.254774 \\ \text { O3 } & 0.950659 & 0.000000 & -0.817897 \\ \text { C4 } & -1.420772 & -0.000030 & -0.646419 \\ \text { C5 } & -1.554929 & -0.000003 & -2.040509 \\ \text { C6 } & -2.826246 & -0.000040 & -2.597366 \\ \text { C7 } & -3.984407 & -0.000091 & -1.822470 \\ \text { C8 } & -3.841488 & -0.000110 & -0.431348 \\ \text { C9 } & -2.572060 & -0.000087 & 0.147775 \\ \text { H10 } & -0.645673 & 0.000055 & -2.631371 \\ \text { H11 } & -4.962414 & -0.000115 & -2.291550 \\ \text { H12 } & -4.733874 & -0.000150 & 0.192555 \\ \text { H13 } & -2.422073 & -0.000119 & 1.223033 \\ \text { Br14 } & -3.025220 & -0.000018 & -4.533152\end{array}$

\section{Compound 21:}

$\begin{array}{rrrr}\text { C1 } & 0.000000 & 0.000000 & 0.000000 \\ \text { O2 } & 0.000000 & 0.000000 & 1.254636 \\ \text { O3 } & 0.949752 & 0.000000 & -0.819803 \\ \text { C4 } & -1.416068 & 0.000005 & -0.648197 \\ \text { C5 } & -1.562823 & 0.000246 & -2.041530 \\ \text { C6 } & -2.821785 & 0.000237 & -2.631183 \\ \text { C7 } & -3.973527 & -0.000002 & -1.819027 \\ \text { C8 } & -3.835665 & -0.000232 & -0.416509 \\ \text { C9 } & -2.566638 & -0.000232 & 0.151189 \\ \text { H10 } & -0.648959 & 0.000440 & -2.627845 \\ \text { H11 } & -2.934806 & 0.000422 & -3.712201 \\ \text { H12 } & -4.727793 & -0.000418 & 0.204362 \\ \text { H13 } & -2.413307 & -0.000424 & 1.226080 \\ \text { C14 } & -5.274856 & -0.000005 & -2.414809 \\ \text { N15 } & -6.335244 & -0.000009 & -2.900321\end{array}$




\section{Compound 22:}

$\begin{array}{rrrr}\text { C1 } & 0.000000 & 0.000000 & 0.000000 \\ \text { O2 } & 0.000000 & 0.000000 & 1.247741 \\ \text { O3 } & 0.945755 & 0.000000 & -0.829564 \\ \text { C4 } & -1.390196 & 0.000529 & -0.730994 \\ \text { H5 } & -1.435665 & 0.885573 & -1.390709 \\ \text { H6 } & -1.436209 & -0.884315 & -1.390939 \\ \text { O7 } & -2.520776 & 0.000765 & 0.148029 \\ \text { C8 } & -3.727113 & 0.001225 & -0.550686 \\ \text { H9 } & -3.846490 & 0.889784 & -1.202048 \\ \text { H10 } & -3.847033 & -0.887091 & -1.202279 \\ \text { H11 } & -4.548055 & 0.001381 & 0.178719\end{array}$

\section{Compound 24:}

$\begin{array}{lrrr}\text { C1 } & 0.000000 & 0.000000 & 0.000000 \\ \text { C2 } & 0.000000 & 0.000000 & 1.344437 \\ \text { H3 } & 0.939189 & 0.000000 & -0.551512 \\ \text { H4 } & -0.956392 & 0.000019 & 1.858183 \\ \text { C5 } & 1.185718 & 0.000005 & 2.196481 \\ \text { O6 } & 1.188368 & 0.000123 & 3.421080 \\ \text { O7 } & 2.383271 & 0.000682 & 1.521643 \\ \text { H8 } & 3.042905 & 0.000873 & 2.233456 \\ \text { C9 } & -1.293422 & 0.000106 & -0.844067 \\ \text { O10 } & -1.080523 & 0.001192 & -2.083430 \\ \text { O11 } & -2.368360 & -0.000273 & -0.195694\end{array}$

\section{Compound 27:}

$\begin{array}{rrrr}\text { C1 } & 0.000000 & 0.000000 & 0.000000 \\ \text { O2 } & 0.000000 & 0.000000 & 1.239722 \\ \text { O3 } & 0.930053 & 0.000000 & -0.847187 \\ \text { C4 } & -1.356114 & 0.000089 & -0.797063 \\ \text { H5 } & -1.418767 & 0.887623 & -1.427825 \\ \text { H6 } & -1.418841 & -0.887395 & -1.427889 \\ \text { Cl7 } & -2.955965 & 0.000121 & 0.174576\end{array}$

\section{Compound 29:}

$\begin{array}{lrrr}\text { C1 } & 0.000000 & 0.000000 & 0.000000 \\ \text { O2 } & 0.000000 & 0.000000 & 1.248007 \\ \text { O3 } & 0.942542 & 0.000000 & -0.834040 \\ \text { C4 } & -1.382908 & 0.000622 & -0.725664 \\ \text { H5 } & -1.449436 & 0.887729 & -1.371974 \\ \text { H6 } & -1.450104 & -0.886263 & -1.372211 \\ \text { F7 } & -2.506571 & 0.000935 & 0.121558\end{array}$

\section{Compound 30:}

$\begin{array}{lrrr}\text { C1 } & 0.000000 & 0.000000 & 0.000000 \\ \text { O2 } & 0.000000 & 0.000000 & 1.241852 \\ \text { O3 } & 0.911132 & 0.000000 & -0.851508 \\ \text { C4 } & -1.463891 & -0.000048 & -0.717803 \\ \text { H5 } & -1.504914 & 0.879007 & -1.370473 \\ \text { H6 } & -1.504768 & -0.878947 & -1.370692 \\ \text { C7 } & -2.625129 & -0.000248 & 0.158624 \\ \text { N8 } & -3.562293 & -0.000410 & 0.850587\end{array}$

\section{Compound 32:}

$\begin{array}{lrrr}\text { C1 } & 1.366181 & -0.006751 & 0.060298 \\ \text { O2 } & 1.518487 & -0.023391 & 1.288393 \\ \text { O3 } & 2.157115 & 0.006249 & -0.908778\end{array}$

\section{Compound 23:}

$\begin{array}{lrrr}\text { C1 } & 0.000000 & 0.000000 & 0.000000 \\ \text { O2 } & 0.000000 & 0.000000 & 1.230269 \\ \text { O3 } & 1.202214 & 0.000000 & -0.629358 \\ \text { C4 } & -1.178442 & 0.000067 & -0.991401 \\ \text { H5 } & -1.126667 & 0.873772 & -1.606915 \\ \text { H6 } & -1.126640 & -0.873531 & -1.607066 \\ \text { C7 } & -2.505957 & -0.000021 & -0.210822 \\ \text { C8 } & -3.536075 & -0.000089 & 0.394887 \\ \text { H9 } & -4.450681 & -0.000150 & 0.932675\end{array}$

\section{Compound 25:}

$\begin{array}{rrrr}\text { C1 } & 0.000000 & 0.000000 & 0.000000 \\ \text { O2 } & 0.000000 & 0.000000 & 1.238980 \\ \text { O3 } & 0.929382 & 0.000000 & -0.848916 \\ \text { C4 } & -1.344684 & 0.000939 & -0.805226 \\ \text { H5 } & -1.427700 & 0.890856 & -1.428350 \\ \text { H6 } & -1.428715 & -0.888627 & -1.428713 \\ \text { Br7 } & -3.090442 & 0.001720 & 0.252852\end{array}$

Compound 26:

$\begin{array}{crrr}\text { C1 } & 0.000000 & 0.000000 & 0.000000 \\ \text { O2 } & 0.000000 & 0.000000 & 1.220472 \\ \text { O3 } & 1.192792 & 0.000000 & -0.678286 \\ \text { H4 } & 1.854152 & 0.105958 & 0.024540 \\ \text { C5 } & -1.176628 & 0.027388 & -0.903424 \\ \text { H6 } & -0.978722 & -0.538666 & -1.817848 \\ \text { H7 } & -2.037215 & -0.404822 & -0.388540 \\ \text { C8 } & -1.578891 & 1.539837 & -1.363362 \\ \text { O9 } & -0.823720 & 2.443545 & -0.955809 \\ \text { O10 } & -2.602591 & 1.556531 & -2.073349 \\ \text { Compound 28: } & & \\ \text { C1 } & 0.000000 & 0.000000 & 0.000000 \\ \text { O2 } & 0.000000 & 0.000000 & 1.250756 \\ \text { O3 } & 0.937658 & 0.000000 & -0.830749 \\ \text { C4 } & -1.431306 & 0.102024 & -0.671502 \\ \text { C5 } & -2.240442 & 1.247466 & -0.092825 \\ \text { H6 } & -1.722798 & 2.187913 & -0.324461 \\ \text { H7 } & -3.259652 & 1.302065 & -0.492146 \\ \text { H8 } & -2.262941 & 1.134465 & 0.993648 \\ \text { H9 } & -1.330380 & 0.154616 & -1.755897 \\ \text { Cl10 } & -2.418709 & -1.479456 & -0.422417\end{array}$

\section{Compound 31:}

$\begin{array}{lrrr}\text { C1 } & 0.000000 & 0.000000 & 0.000000 \\ \text { O2 } & 0.000000 & 0.000000 & 1.244764 \\ \text { O3 } & 0.882579 & 0.000000 & -0.865309 \\ \text { C4 } & -1.549139 & -0.007556 & -0.533033 \\ \text { H5 } & -2.018184 & -0.926580 & -0.185096 \\ \text { H6 } & -2.045539 & 0.877219 & -0.138199 \\ \text { N7 } & -1.684931 & 0.027341 & -1.992953 \\ \text { O8 } & -1.726119 & 1.132329 & -2.550279 \\ \text { O9 } & -1.693405 & -1.048213 & -2.605861 \\ & & & \\ \text { C4 } & -0.138599 & 0.001701 & -0.480820 \\ \text { H5 } & -0.171863 & 0.006382 & -1.566859 \\ \text { Cl6 } & -1.084428 & -1.492994 & 0.025103 \\ \text { Cl7 } & -1.068422 & 1.502468 & 0.036842\end{array}$




\section{Compound 33:}

$\begin{array}{crrr}\text { C1 } & 0.000000 & 0.000000 & 0.000000 \\ \text { O2 } & 0.000000 & 0.000000 & 1.247716 \\ \text { O3 } & 0.915094 & 0.000000 & -0.845527 \\ \text { C4 } & -1.453942 & 0.001787 & -0.661389 \\ \text { O5 } & -1.726754 & 0.002371 & -1.848401 \\ \text { O6 } & -2.480497 & 0.002040 & 0.253322 \\ \text { H7 } & -3.273531 & 0.002961 & -0.311224 \\ \text { Compound 35: } & & \\ \text { C1 } & 0.000000 & 0.000000 & 0.000000 \\ \text { O2 } & 0.000000 & 0.000000 & 1.232068 \\ \text { O3 } & 0.855087 & 0.000000 & -0.889813 \\ \text { C4 } & -1.534639 & -0.000584 & -0.661436 \\ \text { Cl5 } & -2.885188 & -0.000430 & 0.565168 \\ \text { C16 } & -1.763200 & 1.476756 & -1.721345 \\ \text { Cl7 } & -1.762524 & -1.478832 & -1.720216 \\ \text { Compound 37: } & & \\ \text { C1 } & 0.000000 & 0.000000 & 0.000000 \\ \text { C2 } & 0.000000 & 0.000000 & 1.580220 \\ \text { H3 } & 1.041870 & 0.000000 & 1.927448 \\ \text { H4 } & -0.456845 & -0.936525 & 1.927054 \\ \text { H5 } & -0.526987 & 0.843154 & 2.062562 \\ \text { C6 } & -1.513532 & 0.031157 & -0.452543 \\ \text { H7 } & -1.990410 & -0.905186 & -0.133319 \\ \text { H8 } & -2.114301 & 0.875541 & -0.068623 \\ \text { H9 } & -1.547546 & 0.054073 & -1.549996 \\ \text { C10 } & 0.636869 & 1.373073 & -0.453255 \\ \text { H11 } & 0.142096 & 2.284289 & -0.070808 \\ \text { H12 } & 1.687332 & 1.390366 & -0.133482 \\ \text { H13 } & 0.632115 & 1.412328 & -1.550763 \\ \text { O14 } & 0.649404 & -1.040410 & -0.499421 \\ \text { Compound }\end{array}$

\section{Compound 40:}

$\begin{array}{rrrr}\text { C1 } & 0.000000 & 0.543015 & 0.000000 \\ \text { H2 } & -0.387079 & 1.175124 & 0.889021 \\ \text { H3 } & -0.387079 & 1.175124 & -0.889021 \\ \text { O4 } & 1.276325 & 0.247895 & 0.000000 \\ \text { C5 } & -0.975757 & -0.732438 & 0.000000 \\ \text { H6 } & -0.317634 & -1.608866 & 0.000000 \\ \text { H7 } & -1.632132 & -0.794002 & 0.885781 \\ \text { H8 } & -1.632132 & -0.794002 & -0.885781\end{array}$

\section{Compound 41:}

$\begin{array}{rrrr}\text { C1 } & 0.000000 & 0.000000 & 0.000000 \\ \text { H2 } & 0.000000 & 0.000000 & 1.161638 \\ \text { H3 } & 1.144196 & 0.000000 & -0.200581 \\ \text { H4 } & -0.238784 & -1.119006 & -0.200553 \\ \text { O5 } & -0.728587 & 0.900420 & -0.611968\end{array}$

\section{Compound 34:}

$\begin{array}{lrrr}\text { C1 } & 0.000000 & 0.000000 & 0.000000 \\ \text { O2 } & 0.000000 & 0.000000 & 1.248125 \\ \text { O3 } & 0.931070 & 0.000000 & -0.841997 \\ \text { C4 } & -1.399983 & 0.022888 & -0.698051 \\ \text { H5 } & -1.426291 & -0.632942 & -1.580046 \\ \text { F6 } & -2.445459 & -0.342296 & 0.126488 \\ \text { F7 } & -1.699083 & 1.305183 & -1.140796\end{array}$

\section{Compound 36:}

$\begin{array}{lrrr}\text { C1 } & 0.000000 & 0.000000 & 0.000000 \\ \text { O2 } & 0.000000 & 0.000000 & 1.246374 \\ \text { O3 } & 0.910754 & 0.000000 & -0.853353 \\ \text { C4 } & -1.434702 & -0.000079 & -0.662977 \\ \text { F5 } & -2.470805 & -0.000247 & 0.219956 \\ \text { F6 } & -1.634499 & 1.088901 & -1.464874 \\ \text { F7 } & -1.634303 & -1.088960 & -1.465052\end{array}$

Compound 38:

$\begin{array}{cccc}\text { C1 } & 0.000000 & 0.000000 & 0.000000 \\ \text { H2 } & 0.000000 & 0.000000 & 1.100766 \\ \text { H3 } & 1.048468 & 0.000000 & -0.351251 \\ \text { H4 } & -0.472820 & -0.936723 & -0.323247 \\ \text { C5 } & -0.872719 & 1.204679 & -0.516213 \\ \text { H6 } & -0.690164 & 1.180045 & -1.659031 \\ \text { C7 } & -0.149840 & 2.533307 & -0.078541 \\ \text { H8 } & -0.728504 & 3.386073 & -0.457268 \\ \text { H9 } & -0.153859 & 2.601259 & 1.020118 \\ \text { H10 } & 0.892607 & 2.635103 & -0.432948 \\ \text { O11 } & -2.128716 & 1.142569 & -0.123364 \\ \text { Compound 39: } & & \\ \text { C1 } & 0.000000 & 0.000000 & 0.000000 \\ \text { H2 } & 0.000000 & 0.000000 & 1.150000 \\ \text { H3 } & 1.130455 & 0.000000 & -0.211122 \\ \text { O4 } & -0.715920 & -0.925203 & -0.591888 \\ \text { C5 } & -0.414596 & 1.483166 & -0.342622 \\ \text { H6 } & -1.477649 & 1.597215 & -0.080011 \\ \text { H7 } & -0.356850 & 1.596667 & -1.436180 \\ \text { C8 } & 0.421654 & 2.565044 & 0.348266 \\ \text { H9 } & 0.115759 & 3.591154 & 0.094468 \\ \text { H10 } & 0.358769 & 2.464543 & 1.440867 \\ \text { H11 } & 1.483053 & 2.463166 & 0.081893\end{array}$

Compound 42:

$\begin{array}{lrrr}\text { C1 } & 0.000000 & 0.000000 & 0.000000 \\ \text { C2 } & 0.000000 & 0.000000 & 1.594262 \\ \text { H3 } & 1.129748 & 0.000000 & 1.804447 \\ \text { H4 } & -0.276646 & 1.095353 & 1.804447 \\ \text { O5 } & -0.725767 & -0.931859 & 2.137310 \\ \text { F6 } & 1.248406 & -0.166394 & -0.561153 \\ \text { F7 } & -0.764817 & -0.981974 & -0.526875 \\ \text { F8 } & -0.467002 & 1.169664 & -0.561153\end{array}$


Compound 43:

$\begin{array}{rrrr}\text { C1 } & 0.000000 & 0.000000 & 0.000000 \\ \text { C2 } & 0.000000 & 0.000000 & 1.388868 \\ \text { C3 } & 1.216349 & 0.000000 & 2.179573 \\ \text { C4 } & 2.422093 & -0.000365 & 1.369336 \\ \text { C5 } & 2.397035 & -0.000325 & -0.017884 \\ \text { C6 } & 1.190891 & -0.000115 & -0.742433 \\ \text { H7 } & -0.941365 & 0.000083 & -0.550952 \\ \text { H8 } & -0.941300 & 0.000075 & 1.937874 \\ \text { H9 } & 3.372138 & -0.000575 & 1.903275 \\ \text { H10 } & 3.340533 & -0.000466 & -0.572456 \\ \text { O11 } & 1.230072 & -0.000204 & 3.439498 \\ \text { N12 } & 1.134891 & -0.000068 & -2.198671 \\ \text { H13 } & 1.644128 & 0.806978 & -2.560631 \\ \text { H14 } & 1.644620 & -0.806783 & -2.560663 \\ \text { Compound 45: } & & \\ \text { C1 } & 0.000000 & 0.000000 & 0.000000 \\ \text { C2 } & 0.000000 & 0.000000 & 1.391393 \\ \text { C3 } & 1.207151 & 0.000000 & 2.176496 \\ \text { C4 } & 2.415016 & -0.000331 & 1.366190 \\ \text { C5 } & 2.390106 & -0.000327 & -0.020001 \\ \text { C6 } & 1.187215 & -0.000120 & -0.753757 \\ \text { H7 } & -0.955373 & 0.000092 & -0.532644 \\ \text { H8 } & -0.942608 & 0.000101 & 1.938333 \\ \text { H9 } & 3.365626 & -0.000513 & 1.899387 \\ \text { H10 } & 3.337821 & -0.000491 & -0.566339 \\ \text { O11 } & 1.221839 & -0.000116 & 3.436410 \\ \text { C12 } & 1.175126 & -0.000057 & -2.264466 \\ \text { H13 } & 1.674332 & 0.880191 & -2.701933 \\ \text { H14 } & 1.675046 & -0.879880 & -2.701947 \\ \text { H15 } & 0.146207 & -0.000461 & -2.645850 \\ \text { Compound }\end{array}$

\section{Compound 48:}

$\begin{array}{rrrr}\text { C1 } & 0.000000 & 0.000000 & 0.000000 \\ \text { C2 } & 0.000000 & 0.000000 & 1.391889 \\ \text { C3 } & 1.208900 & 0.000000 & 2.192551 \\ \text { C4 } & 2.416139 & -0.000298 & 1.391646 \\ \text { C5 } & 2.403780 & -0.000328 & -0.001965 \\ \text { C6 } & 1.201337 & -0.000160 & -0.714527 \\ \text { H7 } & -0.937031 & 0.000088 & -0.557008 \\ \text { H8 } & -0.945546 & 0.000134 & 1.932921 \\ \text { H9 } & 3.364622 & -0.000415 & 1.927089 \\ \text { H10 } & 3.349958 & -0.000525 & -0.553574 \\ \text { O11 } & 1.209638 & -0.000043 & 3.452551 \\ \text { O12 } & 1.156453 & -0.000239 & -2.122585 \\ \text { H13 } & 2.076154 & 0.000223 & -2.414821\end{array}$

\section{Compound 49:}

$\begin{array}{lrrr}\text { C1 } & 0.000000 & 0.000000 & 0.000000 \\ \text { C2 } & 0.000000 & 0.000000 & 1.391721 \\ \text { C3 } & 1.214455 & 0.000000 & 2.187187 \\ \text { C4 } & 2.425112 & -0.000486 & 1.385881 \\ \text { C5 } & 2.418434 & -0.000508 & -0.005804 \\ \text { C6 } & 1.207549 & -0.000211 & -0.693296\end{array}$

\section{Compound 44:}

$\begin{array}{lrrr}\text { C1 } & -0.006295 & 1.368094 & 0.000010 \\ \text { C2 } & -1.379917 & 1.171397 & 0.000034 \\ \text { C3 } & -1.996954 & -0.143135 & -0.000445 \\ \text { C4 } & -1.029067 & -1.217972 & -0.000144 \\ \text { C5 } & 0.351529 & -1.013133 & -0.000155 \\ \text { C6 } & 0.879957 & 0.282690 & -0.000185 \\ \text { H7 } & 0.414456 & 2.373824 & 0.000158 \\ \text { H8 } & -2.051831 & 2.028766 & 0.000351 \\ \text { H9 } & -1.421402 & -2.234025 & 0.000052 \\ \text { H10 } & 1.010746 & -1.879416 & -0.000115 \\ \text { O11 } & -3.244116 & -0.322540 & 0.000400 \\ \text { O12 } & 2.253310 & 0.599080 & -0.000208 \\ \text { C13 } & 3.141586 & -0.479931 & 0.000320 \\ \text { H14 } & 3.027010 & -1.123770 & 0.889284 \\ \text { H15 } & 3.027741 & -1.124085 & -0.888511 \\ \text { H16 } & 4.154690 & -0.061676 & 0.000631\end{array}$

\section{Compound 46:}

$\begin{array}{rrrr}\text { C1 } & 0.000000 & 0.000000 & 0.000000 \\ \text { C2 } & 0.000000 & 0.000000 & 1.380000 \\ \text { C3 } & 1.222228 & 0.000000 & 2.163299 \\ \text { C4 } & 2.424185 & -0.000384 & 1.352060 \\ \text { C5 } & 2.414827 & -0.000394 & -0.040289 \\ \text { C6 } & 1.195916 & -0.000136 & -0.741628 \\ \text { H7 } & -0.952656 & 0.000119 & -0.535277 \\ \text { H8 } & -0.937492 & 0.000099 & 1.935571 \\ \text { H9 } & 3.373470 & -0.000577 & 1.890181 \\ \text { H10 } & 1.183489 & -0.000142 & -1.830349 \\ \text { C11 } & 3.725426 & -0.000577 & -0.806538 \\ \text { H12 } & 4.338381 & 0.879517 & -0.567800 \\ \text { H13 } & 4.338159 & -0.880801 & -0.567722 \\ \text { H14 } & 3.557895 & -0.000596 & -1.890374 \\ \text { O15 } & 1.236768 & -0.000191 & 3.423215\end{array}$

\section{Compound 47:}

$\begin{array}{rrrr}\text { C1 } & 0.000000 & 0.000000 & 0.000000 \\ \text { C2 } & 0.000000 & 0.000000 & 1.404775 \\ \text { C3 } & 1.246318 & 0.000000 & 2.052877 \\ \text { C4 } & 2.439070 & -0.000223 & 1.342736 \\ \text { C5 } & 2.492144 & -0.000556 & -0.108218 \\ \text { C6 } & 1.180335 & -0.000212 & -0.730569 \\ \text { H7 } & -0.954424 & 0.000110 & -0.532750 \\ \text { H8 } & -0.930153 & 0.000273 & 1.969467 \\ \text { H9 } & 1.278660 & 0.000094 & 3.145444 \\ \text { H10 } & 3.394625 & -0.000203 & 1.867112 \\ \text { H11 } & 1.156019 & -0.000189 & -1.820277 \\ \text { O12 } & 3.569180 & -0.000476 & -0.762124\end{array}$

$\begin{array}{rrrr}\text { H7 } & -0.932368 & 0.000136 & -0.563920 \\ \text { H8 } & -0.943482 & 0.000148 & 1.935372 \\ \text { H9 } & 3.371163 & -0.000727 & 1.925048 \\ \text { H10 } & 3.348065 & -0.000751 & -0.574227 \\ \text { O11 } & 1.217514 & -0.000240 & 3.447183 \\ \text { F12 } & 1.204240 & -0.000216 & -2.077643\end{array}$


Compound 50:

$\begin{array}{lrrr}\text { C1 } & 0.000000 & 0.000000 & 0.000000 \\ \text { C2 } & 0.000000 & 0.000000 & 1.380000 \\ \text { C3 } & 1.221460 & 0.000000 & 2.164966 \\ \text { C4 } & 2.422581 & 0.000466 & 1.352681 \\ \text { C5 } & 2.402795 & 0.000475 & -0.038626 \\ \text { C6 } & 1.193613 & 0.000191 & -0.746802 \\ \text { H7 } & -0.953823 & -0.000131 & -0.532680 \\ \text { H8 } & -0.937512 & -0.000088 & 1.935215 \\ \text { H9 } & 3.376679 & 0.000764 & 1.882703 \\ \text { H10 } & 1.203384 & 0.000203 & -1.832647 \\ \text { O11 } & 1.237552 & 0.000392 & 3.424863 \\ \text { N12 } & 3.647109 & 0.000773 & -0.817974 \\ \text { H13 } & 4.199063 & 0.802919 & -0.510342 \\ \text { H14 } & 4.199781 & -0.800681 & -0.509823 \\ \text { Compound 52: } & & \\ \text { C1 } & 0.000000 & 0.000000 & 0.000000 \\ \text { C2 } & 0.000000 & 0.000000 & 1.385583 \\ \text { C3 } & 1.227849 & 0.000000 & 2.162694 \\ \text { C4 } & 2.427052 & -0.000293 & 1.346517 \\ \text { C5 } & 2.390285 & -0.000299 & -0.040658 \\ \text { C6 } & 1.185618 & -0.000126 & -0.755914 \\ \text { H7 } & -0.954527 & 0.000086 & -0.530698 \\ \text { H8 } & -0.935588 & 0.000139 & 1.942659 \\ \text { H9 } & 3.386061 & -0.000397 & 1.869746 \\ \text { H10 } & 1.189607 & -0.000166 & -1.841322 \\ \text { O11 } & 1.252666 & -0.000033 & 3.427155 \\ \text { O12 } & 3.571748 & -0.000469 & -0.785595 \\ \text { H13 } & 4.288272 & -0.000403 & -0.137581 \\ \text { Comp }\end{array}$

\section{Compound 54:}

$\begin{array}{crrr}\text { C1 } & 0.000000 & 0.000000 & 0.000000 \\ \text { C2 } & 0.000000 & 0.000000 & 1.387055 \\ \text { C3 } & 1.214966 & 0.000000 & 2.183869 \\ \text { C4 } & 2.428624 & -0.000486 & 1.384997 \\ \text { C5 } & 2.426303 & -0.000512 & -0.002038 \\ \text { C6 } & 1.212556 & -0.000187 & -0.696425 \\ \text { H7 } & -0.938196 & 0.000161 & -0.551214 \\ \text { H8 } & -0.944496 & 0.000143 & 1.928577 \\ \text { H9 } & 3.373998 & -0.000727 & 1.924985 \\ \text { H10 } & 3.363556 & -0.000735 & -0.554854 \\ \text { O11 } & 1.216056 & -0.000235 & 3.443869 \\ \text { Br12 } & 1.210938 & -0.000235 & -2.641820 \\ \text { Compound 56: } & & \\ \text { C1 } & 0.000000 & 0.000000 & 0.000000 \\ \text { C2 } & 0.000000 & 0.000000 & 1.380000 \\ \text { C3 } & 1.225883 & 0.000000 & 2.163005 \\ \text { C4 } & 2.433615 & 0.000615 & 1.360582 \\ \text { C5 } & 2.369083 & 0.000633 & -0.017225 \\ \text { C6 } & 1.186119 & 0.000270 & -0.758775 \\ \text { H7 } & -0.952435 & -0.000156 & -0.532900 \\ \text { H8 } & -0.936967 & -0.000123 & 1.933925 \\ \text { H9 } & 3.384704 & 0.000960 & 1.881609 \\ \text { H10 } & 1.188025 & 0.000323 & -1.841496 \\ \text { O11 } & 1.250740 & 0.000478 & 3.422759 \\ \text { Br12 } & 4.059164 & 0.001186 & -1.036440 \\ & & & \end{array}$

\section{Compound 51:}

$\begin{array}{lrrr}\text { C1 } & 0.000000 & 0.000000 & 0.000000 \\ \text { C2 } & 0.000000 & 0.000000 & 1.380000 \\ \text { C3 } & 1.228318 & 0.000000 & 2.155781 \\ \text { C4 } & 2.423408 & -0.000056 & 1.352438 \\ \text { C5 } & 2.395030 & -0.000039 & -0.037355 \\ \text { C6 } & 1.188787 & -0.000022 & -0.758545 \\ \text { H7 } & -0.951982 & 0.000028 & -0.535358 \\ \text { H8 } & -0.936287 & -0.000014 & 1.936098 \\ \text { H9 } & 3.385517 & -0.000166 & 1.858694 \\ \text { H10 } & 1.154867 & 0.000210 & -1.841669 \\ \text { O11 } & 1.242959 & -0.000013 & 3.419943 \\ \text { O12 } & 3.649082 & 0.000079 & -0.664326 \\ \text { C13 } & 3.668805 & -0.002348 & -2.066190 \\ \text { H14 } & 3.179360 & -0.891820 & -2.493947 \\ \text { H15 } & 3.180533 & 0.886278 & -2.497101 \\ \text { H16 } & 4.722224 & -0.003491 & -2.367606\end{array}$

\section{Compound 53:}

$\begin{array}{lrrr}\text { C1 } & 0.000000 & 0.000000 & 0.000000 \\ \text { C2 } & 0.000000 & 0.000000 & 1.387527 \\ \text { C3 } & 1.214473 & 0.000000 & 2.184443 \\ \text { C4 } & 2.427121 & -0.000495 & 1.384687 \\ \text { C5 } & 2.423903 & -0.000514 & -0.002819 \\ \text { C6 } & 1.211129 & -0.000190 & -0.699246 \\ \text { H7 } & -0.938501 & 0.000160 & -0.551528 \\ \text { H8 } & -0.944439 & 0.000136 & 1.929168 \\ \text { H9 } & 3.372788 & -0.000748 & 1.924180 \\ \text { H10 } & 3.361104 & -0.000741 & -0.556553 \\ \text { O11 } & 1.215971 & -0.000258 & 3.444442 \\ \text { Cl12 } & 1.209052 & -0.000224 & -2.494801\end{array}$

\section{Compound 55:}

$\begin{array}{lrrr}\text { C1 } & 0.000000 & 0.000000 & 0.000000 \\ \text { C2 } & 0.000000 & 0.000000 & 1.385297 \\ \text { C3 } & 1.224156 & 0.000000 & 2.171016 \\ \text { C4 } & 2.433563 & 0.000647 & 1.364298 \\ \text { C5 } & 2.374333 & 0.000691 & -0.012680 \\ \text { C6 } & 1.190020 & 0.000251 & -0.751150 \\ \text { H7 } & -0.953076 & -0.000192 & -0.532395 \\ \text { H8 } & -0.937080 & -0.000065 & 1.939617 \\ \text { H9 } & 3.396519 & 0.001068 & 1.867517 \\ \text { H10 } & 1.208452 & 0.000237 & -1.835679 \\ \text { O11 } & 1.237776 & 0.000616 & 3.433658 \\ \text { F12 } & 3.560593 & 0.000981 & -0.718728\end{array}$

\section{Compound 57:}

$\begin{array}{rrrr}\text { C1 } & 0.000000 & 0.000000 & 0.000000 \\ \text { C2 } & 0.000000 & 0.000000 & 1.380000 \\ \text { C3 } & 1.222453 & 0.000000 & 2.162714 \\ \text { C4 } & 2.431717 & -0.001073 & 1.361539 \\ \text { C5 } & 2.372617 & -0.001111 & -0.017824 \\ \text { C6 } & 1.186893 & -0.000412 & -0.757382 \\ \text { H7 } & -0.952130 & 0.000293 & -0.533470 \\ \text { H8 } & -0.937364 & 0.000090 & 1.933198 \\ \text { H9 } & 3.383488 & -0.001748 & 1.882375 \\ \text { H10 } & 1.190288 & -0.000448 & -1.840710 \\ \text { O11 } & 1.245487 & -0.001030 & 3.422503 \\ \text { Cl12 } & 3.929712 & -0.001926 & -0.950692\end{array}$




\section{Compound 58:}

C1 0.000000

C2 $\quad 0.000000$

C3 1.215362

C4 2.425852

C5 2.400651

C6 1.188398

H7 -0.951851

$\mathrm{H} 8 \quad-0.939720$

H9 3.375709

$\mathrm{H} 10 \quad 1.191858$

O11 1.222327

C12 3.634968

N13 4.640015

\section{Compound 61:}

$\begin{array}{lrrr}\text { C1 } & 0.000000 & 0.000000 & 0.000000 \\ \text { C2 } & 0.000000 & 0.000000 & 1.370000 \\ \text { C3 } & 1.228674 & 0.000000 & 2.159245 \\ \text { C4 } & 2.452779 & -0.000066 & 1.363528 \\ \text { C5 } & 2.444930 & -0.000066 & -0.007565 \\ \text { C6 } & 1.220411 & -0.000027 & -0.719969 \\ \text { H7 } & -0.923874 & 0.000018 & -0.568536 \\ \text { H8 } & -0.935016 & 0.000001 & 1.925622 \\ \text { H9 } & 3.390962 & -0.000112 & 1.913787 \\ \text { H10 } & 3.365528 & -0.000095 & -0.581391 \\ \text { O11 } & 1.232261 & -0.000039 & 3.409239 \\ \text { N12 } & 1.216365 & -0.000024 & -2.131210 \\ \text { O13 } & 2.309354 & -0.000157 & -2.747620 \\ \text { O14 } & 0.119859 & -0.000097 & -2.741342\end{array}$

\section{Compound 62:}

$\begin{array}{lrrr}\text { C1 } & 0.000000 & 0.000000 & 0.000000 \\ \text { C2 } & 0.000000 & 0.000000 & 1.364505 \\ \text { C3 } & 1.373795 & 0.000000 & 1.779508 \\ \text { C4 } & 2.111081 & -0.004207 & 0.637244 \\ \text { O5 } & 1.294345 & -0.003265 & -0.457369 \\ \text { H6 } & -0.874819 & 0.002731 & 1.999247 \\ \text { H7 } & 1.755045 & 0.003080 & 2.790485 \\ \text { H8 } & 3.170699 & -0.006156 & 0.434353 \\ \text { C9 } & -1.074639 & -0.034040 & -1.041776 \\ \text { H10 } & -0.962616 & 0.823338 & -1.716573 \\ \text { H11 } & -2.038665 & 0.087722 & -0.537503 \\ \text { N12 } & -1.129220 & -1.245837 & -1.874683 \\ \text { H13 } & -0.237668 & -1.352373 & -2.354627 \\ \text { H14 } & -1.212916 & -2.060392 & -1.268886\end{array}$

\section{Compound 59:}

C1 0.000000

C2 $\quad 0.000000$

C3 1.213171

C4 2.425877

C5 2.378832

C6 1.185382

$\mathrm{H} 7 \quad-0.951316$

H8 -0.940000

H9 3.381149

$\mathrm{H} 10 \quad 1.204714$

O11 1.218809

N12 3.642085

$013 \quad 4.710802$

O14 3.601897

Compound 60:

$\begin{array}{cccc}\text { C1 } & 0.000000 & 0.000000 & 0.000000 \\ \text { C2 } & 0.000000 & 0.000000 & 1.370000 \\ \text { C3 } & 1.227447 & 0.000000 & 2.160295 \\ \text { C4 } & 2.437751 & 0.000102 & 1.361757 \\ \text { C5 } & 2.429403 & 0.000101 & -0.010457 \\ \text { C6 } & 1.211507 & 0.000032 & -0.745976 \\ \text { H7 } & -0.943072 & -0.000035 & -0.544955 \\ \text { H8 } & -0.937550 & 0.000011 & 1.922378 \\ \text { H9 } & 3.380002 & 0.000189 & 1.906078 \\ \text { H10 } & 3.367743 & 0.000145 & -0.563519 \\ \text { O11 } & 1.232841 & 0.000210 & 3.410283 \\ \text { C12 } & 1.205425 & 0.000033 & -2.161829 \\ \text { N13 } & 1.200403 & 0.000049 & -3.334148\end{array}$

$0.000000 \quad 0.000000$

$0.000000 \quad 1.389336$

$0.000000 \quad 2.183513$

$0.000018 \quad 1.385274$

$0.000024-0.002934$

$0.000015-0.743086$

$-0.000010 \quad-0.533034$

0.0000001 .938233

$0.000038 \quad 1.895031$

$\begin{array}{ll}0.000016 & -1.823547\end{array}$

$0.000096 \quad 3.433500$

$0.000037-0.748273$

$0.000272-0.123666$

$0.000000 \quad 0.000000$

(1)

$\begin{array}{ll}0.000101 & -0.010457\end{array}$

$0.000032-0.745976$

$\begin{array}{ll}0.000032 & -0.745976 \\ 0.000035 & -0.544955\end{array}$

$\begin{array}{ll}0.000189 & 1.906078\end{array}$

$0.000145-0.563519$

$0.000033-2.161829$

$0.000049-3.334148$
$0.000419-1.988745$

Part S2. Molecular orbital energy order

S2A) 6-31G(d,p)

\begin{tabular}{ccccccc}
\hline \multirow{2}{*}{ Compound } & \multicolumn{3}{c}{ Hartree-Fock } & \multicolumn{3}{c}{ DFT/B3LYP } \\
& HOMO & $\boldsymbol{p}_{\boldsymbol{z}}$ type & $\boldsymbol{p}_{\boldsymbol{x}} \boldsymbol{p}_{\boldsymbol{y}}$ type & HOMO & $\boldsymbol{p}_{\boldsymbol{z}}$ type & $\boldsymbol{p}_{\boldsymbol{x}} \boldsymbol{p}_{\boldsymbol{y}}$ type \\
\hline $\mathbf{1}$ & 28 & 27 & 26 & 28 & 26 & 28 \\
$\mathbf{2}$ & 20 & 20 & 18 & 20 & 18 & 20
\end{tabular}




\begin{tabular}{|c|c|c|c|c|c|c|}
\hline 3 & 24 & 24 & 22 & 24 & 22 & 24 \\
\hline 4 & 16 & 16 & 14 & 16 & 14 & 16 \\
\hline 5 & 36 & 35 & 32 & 36 & 34 & 36 \\
\hline 6 & 23 & 22 & 21 & 23 & 21 & 23 \\
\hline 7 & 36 & 35 & 34 & 36 & 34 & 36 \\
\hline 8 & 36 & 36 & 32 & 36 & 34 & 36 \\
\hline 9 & 31 & 31 & 29 & 31 & 29 & 31 \\
\hline 10 & 32 & 32 & 28 & 32 & 30 & 32 \\
\hline 11 & 36 & 35 & 33 & 36 & 34 & 36 \\
\hline 12 & 28 & 27 & 26 & 28 & 26 & 28 \\
\hline 13 & 40 & 40 & 37 & 40 & 38 & 40 \\
\hline 14 & 49 & 49 & 46 & 49 & 47 & 49 \\
\hline 15 & 36 & 36 & 32 & 36 & 34 & 36 \\
\hline 16 & 40 & 40 & 36 & 40 & 38 & 40 \\
\hline 17 & 20 & 20 & 18 & 20 & 18 & 20 \\
\hline 18 & 49 & 49 & 45 & 49 & 47 & 49 \\
\hline 19 & 12 & 11 & 10 & 12 & 10 & 12 \\
\hline 20 & 38 & 38 & 35 & 38 & 36 & 38 \\
\hline 21 & 38 & 38 & 35 & 38 & 36 & 38 \\
\hline 22 & 24 & 24 & 22 & 24 & 22 & 24 \\
\hline 23 & 22 & 21 & 20 & 22 & 20 & 22 \\
\hline 24 & 30 & 30 & 28 & 30 & 28 & 30 \\
\hline 25 & 33 & 32 & 31 & 33 & 31 & 33 \\
\hline 26 & 27 & 26 & 25 & 27 & 25 & 27 \\
\hline 27 & 24 & 24 & 22 & 24 & 22 & 24 \\
\hline 28 & 28 & 28 & 26 & 28 & 26 & 28 \\
\hline 29 & 20 & 20 & 18 & 20 & 18 & 20 \\
\hline 30 & 22 & 22 & 20 & 22 & 20 & 22 \\
\hline 31 & 27 & 27 & 25 & 27 & 25 & 27 \\
\hline 32 & 32 & 32 & 30 & 32 & 30 & 32 \\
\hline 33 & 23 & 23 & 21 & 23 & 21 & 23 \\
\hline 34 & 24 & 24 & 22 & 24 & 22 & 24 \\
\hline 35 & 40 & 39 & 38 & 40 & 38 & 40 \\
\hline 36 & 28 & 28 & 26 & 28 & 26 & 28 \\
\hline 37 & 21 & 21 & 20 & 21 & 20 & 21 \\
\hline 38 & 17 & 17 & 16 & 17 & 17 & 16 \\
\hline 39 & 17 & 17 & 16 & 17 & 17 & 16 \\
\hline 40 & 13 & 13 & 12 & 13 & 13 & 12 \\
\hline 41 & 9 & 9 & 8 & 9 & 8 & 9 \\
\hline 42 & 25 & 25 & 24 & 25 & 25 & 24 \\
\hline 43 & 29 & 29 & 27 & 29 & 29 & 28 \\
\hline
\end{tabular}




\begin{tabular}{|c|c|c|c|c|c|c|}
\hline 44 & 33 & 33 & 31 & 33 & 33 & 32 \\
\hline 45 & 29 & 29 & 27 & 29 & 29 & 28 \\
\hline 46 & 29 & 29 & 27 & 29 & 29 & 28 \\
\hline 47 & 25 & 25 & 23 & 25 & 25 & 24 \\
\hline 48 & 29 & 29 & 27 & 29 & 29 & 28 \\
\hline 49 & 29 & 29 & 27 & 29 & 29 & 28 \\
\hline 50 & 29 & 29 & 27 & 29 & 29 & 28 \\
\hline 51 & 33 & 33 & 31 & 33 & 33 & 32 \\
\hline 52 & 29 & 29 & 27 & 29 & 29 & 28 \\
\hline 53 & 33 & 33 & 31 & 33 & 33 & 32 \\
\hline 54 & 42 & 42 & 40 & 42 & 42 & 41 \\
\hline 55 & 29 & 29 & 27 & 29 & 29 & 28 \\
\hline 56 & 42 & 42 & 40 & 42 & 42 & 41 \\
\hline 57 & 33 & 33 & 31 & 33 & 33 & 32 \\
\hline 58 & 31 & 31 & 29 & 31 & 31 & 30 \\
\hline 59 & 36 & 36 & 34 & 36 & 36 & 35 \\
\hline 60 & 31 & 31 & 29 & 31 & 31 & 30 \\
\hline 61 & 36 & 36 & 34 & 36 & 36 & 35 \\
\hline \multicolumn{7}{|c|}{ S2B) PCM/6-31G(d,p) } \\
\hline \multirow{2}{*}{ Compound } & \multicolumn{3}{|c|}{ Hartree-Fock } & \multicolumn{3}{|c|}{ DFT/B3LYP } \\
\hline & HOMO & $p_{z}$ type & $p_{x} p_{y}$ type & HOMO & $p_{z}$ type & $p_{x} p_{y}$ type \\
\hline 1 & 28 & 27 & 26 & 28 & 26 & 27 \\
\hline 2 & 20 & 19 & 18 & 20 & 18 & 19 \\
\hline 3 & 24 & 23 & 22 & 24 & 22 & 23 \\
\hline 4 & 16 & 15 & 14 & 16 & 14 & 15 \\
\hline 5 & 36 & 33 & 32 & 36 & 33 & 36 \\
\hline 6 & 23 & 21 & 20 & 23 & 21 & 22 \\
\hline 7 & 36 & 33 & 32 & 36 & 34 & 35 \\
\hline 8 & 36 & 33 & 32 & 36 & 33 & 36 \\
\hline 9 & 31 & 30 & 29 & 31 & 29 & 30 \\
\hline 10 & 32 & 29 & 28 & 32 & 30 & 32 \\
\hline 11 & 36 & 33 & 32 & 36 & 33 & 36 \\
\hline 12 & 28 & 27 & 26 & 28 & 26 & 27 \\
\hline 13 & 40 & 37 & 36 & 40 & 37 & 40 \\
\hline 14 & 49 & 46 & 45 & 49 & 46 & 49 \\
\hline 15 & 36 & 33 & 32 & 36 & 33 & 36 \\
\hline 16 & 40 & 37 & 36 & 40 & 37 & 40 \\
\hline 17 & 20 & 19 & 18 & 20 & 18 & 19 \\
\hline 18 & 49 & 46 & 45 & 49 & 46 & 48 \\
\hline 19 & 12 & 11 & 10 & 12 & 10 & 11 \\
\hline
\end{tabular}




\begin{tabular}{|c|c|c|c|c|c|c|}
\hline 20 & 38 & 35 & 34 & 38 & 36 & 38 \\
\hline 21 & 38 & 35 & 34 & 38 & 36 & 38 \\
\hline 22 & 24 & 23 & 22 & 24 & 22 & 23 \\
\hline 23 & 22 & 20 & 18 & 22 & 20 & 21 \\
\hline 24 & 30 & 29 & 27 & 30 & 28 & 30 \\
\hline 25 & 33 & 31 & 29 & 33 & 31 & 32 \\
\hline 26 & 27 & 26 & 25 & 27 & 25 & 26 \\
\hline 27 & 24 & 23 & 22 & 24 & 22 & 23 \\
\hline 28 & 28 & 27 & 26 & 28 & 26 & 27 \\
\hline 29 & 20 & 19 & 18 & 20 & 18 & 19 \\
\hline 30 & 22 & 21 & 20 & 22 & 20 & 21 \\
\hline 31 & 27 & 26 & 25 & 27 & 25 & 26 \\
\hline 32 & 32 & 31 & 30 & 32 & 30 & 31 \\
\hline 33 & 23 & 22 & 21 & 23 & 21 & 22 \\
\hline 34 & 24 & 23 & 22 & 24 & 22 & 23 \\
\hline 35 & 40 & 39 & 38 & 40 & 38 & 39 \\
\hline 36 & 28 & 28 & 26 & 28 & 26 & 27 \\
\hline 37 & 21 & 21 & 20 & 21 & 20 & 21 \\
\hline 38 & 17 & 17 & 16 & 17 & 17 & 16 \\
\hline 39 & 17 & 17 & 16 & 17 & 17 & 16 \\
\hline 40 & 13 & 13 & 12 & 13 & 13 & 12 \\
\hline 41 & 9 & 9 & 8 & 9 & 8 & 9 \\
\hline 42 & 25 & 25 & 24 & 25 & 25 & 24 \\
\hline 43 & 29 & 29 & 27 & 29 & 29 & 28 \\
\hline 44 & 33 & 33 & 31 & 33 & 33 & 32 \\
\hline 45 & 29 & 29 & 27 & 29 & 29 & 28 \\
\hline 46 & 29 & 29 & 27 & 29 & 29 & 28 \\
\hline 47 & 25 & 25 & 23 & 25 & 25 & 24 \\
\hline 48 & 29 & 29 & 27 & 29 & 29 & 28 \\
\hline 49 & 29 & 29 & 27 & 29 & 29 & 28 \\
\hline 50 & 29 & 29 & 27 & 29 & 29 & 28 \\
\hline 51 & 33 & 33 & 31 & 33 & 33 & 31 \\
\hline 52 & 29 & 29 & 27 & 29 & 29 & 27 \\
\hline 53 & 33 & 33 & 31 & 33 & 33 & 32 \\
\hline 54 & 42 & 42 & 40 & 42 & 42 & 41 \\
\hline 55 & 29 & 29 & 27 & 29 & 29 & 28 \\
\hline 56 & 42 & 42 & 40 & 42 & 42 & 41 \\
\hline 57 & 33 & 33 & 31 & 33 & 33 & 32 \\
\hline 58 & 31 & 31 & 29 & 31 & 31 & 30 \\
\hline 59 & 36 & 36 & 34 & 36 & 36 & 35 \\
\hline 60 & 31 & 31 & 29 & 31 & 31 & 30 \\
\hline
\end{tabular}




\begin{tabular}{|c|c|c|c|c|c|c|}
\hline 61 & 36 & 36 & 34 & 36 & 36 & 35 \\
\hline \multicolumn{7}{|c|}{ S2C) PCM/6-31+G(d,p) } \\
\hline \multirow{2}{*}{ Compound } & \multicolumn{3}{|c|}{ Hartree-Fock } & \multicolumn{3}{|c|}{ DFT/B3LYP } \\
\hline & HOMO & $p_{z}$ type & $p_{x} p_{y}$ type & HOMO & $p_{z}$ type & $p_{x} p_{y}$ type \\
\hline 1 & 28 & 27 & 26 & 28 & 26 & 27 \\
\hline 2 & 20 & 19 & 18 & 20 & 18 & 19 \\
\hline 3 & 24 & 23 & 22 & 24 & 22 & 23 \\
\hline 4 & 16 & 15 & 14 & 16 & 14 & 15 \\
\hline 5 & 36 & 33 & 32 & 36 & 32 & 33 \\
\hline 6 & 23 & 21 & 20 & 23 & 20 & 22 \\
\hline 7 & 36 & 33 & 32 & 36 & 32 & 35 \\
\hline 8 & 36 & 33 & 32 & 36 & 32 & 33 \\
\hline 9 & 31 & 30 & 29 & 31 & 29 & 30 \\
\hline 10 & 32 & 29 & 28 & 32 & 28 & 29 \\
\hline 11 & 36 & 33 & 32 & 36 & 32 & 35 \\
\hline 12 & 28 & 27 & 26 & 28 & 26 & 27 \\
\hline 13 & 40 & 37 & 36 & 40 & 36 & 38 \\
\hline 14 & 49 & 45 & 44 & 49 & 45 & 48 \\
\hline 15 & 36 & 33 & 32 & 36 & 32 & 35 \\
\hline 16 & 40 & 37 & 36 & 40 & 36 & 38 \\
\hline 17 & 20 & 19 & 18 & 20 & 18 & 19 \\
\hline 18 & 49 & 45 & 43 & 49 & 45 & 47 \\
\hline 19 & 12 & 11 & 10 & 12 & 10 & 11 \\
\hline 20 & 38 & 35 & 34 & 38 & 34 & 37 \\
\hline 21 & 38 & 35 & 34 & 38 & 34 & 38 \\
\hline 22 & 24 & 23 & 21 & 24 & 21 & 22 \\
\hline 23 & 22 & 19 & 18 & 22 & 19 & 21 \\
\hline 24 & 30 & 29 & 27 & 30 & 28 & 29 \\
\hline 25 & 33 & 30 & 29 & 33 & 29 & 32 \\
\hline 26 & 27 & 26 & 25 & 27 & 25 & 26 \\
\hline 27 & 24 & 23 & 21 & 24 & 22 & 23 \\
\hline 28 & 28 & 27 & 25 & 28 & 26 & 27 \\
\hline 29 & 20 & 19 & 18 & 20 & 18 & 19 \\
\hline 30 & 22 & 21 & 20 & 22 & 20 & 21 \\
\hline 31 & 27 & 26 & 25 & 27 & 25 & 26 \\
\hline 32 & 32 & 31 & 29 & 32 & 30 & 31 \\
\hline 33 & 23 & 22 & 21 & 23 & 21 & 22 \\
\hline 34 & 24 & 23 & 22 & 24 & 22 & 23 \\
\hline 35 & 40 & 39 & 38 & 40 & 38 & 39 \\
\hline 36 & 28 & 28 & 26 & 28 & 26 & 27 \\
\hline
\end{tabular}




\begin{tabular}{|c|c|c|c|c|c|c|}
\hline 37 & 21 & 21 & 20 & 21 & 20 & 21 \\
\hline 38 & 17 & 17 & 16 & 17 & 17 & 16 \\
\hline 39 & 17 & 17 & 16 & 17 & 17 & 16 \\
\hline 40 & 13 & 13 & 12 & 13 & 13 & 12 \\
\hline 41 & 9 & 9 & 8 & 9 & 8 & 9 \\
\hline 42 & 25 & 25 & 24 & 25 & 25 & 24 \\
\hline 43 & 29 & 29 & 27 & 29 & 29 & 28 \\
\hline 44 & 33 & 33 & 31 & 33 & 33 & 32 \\
\hline 45 & 29 & 29 & 27 & 29 & 29 & 28 \\
\hline 46 & 29 & 29 & 27 & 29 & 29 & 27 \\
\hline 47 & 25 & 25 & 23 & 25 & 25 & 24 \\
\hline 48 & 29 & 29 & 27 & 29 & 29 & 28 \\
\hline 49 & 29 & 29 & 27 & 29 & 29 & 28 \\
\hline 50 & 29 & 29 & 27 & 29 & 29 & 28 \\
\hline 51 & 33 & 33 & 31 & 33 & 33 & 31 \\
\hline 52 & 29 & 29 & 27 & 29 & 29 & 27 \\
\hline 53 & 33 & 33 & 31 & 33 & 33 & 32 \\
\hline 54 & 42 & 42 & 40 & 42 & 42 & 41 \\
\hline 55 & 29 & 29 & 27 & 29 & 29 & 27 \\
\hline 56 & 42 & 42 & 40 & 42 & 42 & 41 \\
\hline 57 & 33 & 33 & 31 & 33 & 33 & 32 \\
\hline 58 & 31 & 31 & 29 & 31 & 31 & 30 \\
\hline 59 & 36 & 36 & 34 & 36 & 36 & 35 \\
\hline 60 & 31 & 31 & 29 & 31 & 31 & 30 \\
\hline 61 & 36 & 36 & 34 & 36 & 36 & 35 \\
\hline \multicolumn{7}{|c|}{ S2D) $\operatorname{cosMO} / 6-31 G(d, p)$} \\
\hline \multirow{2}{*}{ Compound } & \multicolumn{3}{|c|}{ Hartree-Fock } & \multicolumn{3}{|c|}{ DFT/B3LYP } \\
\hline & HOMO & $p_{z}$ type & $p_{x} p_{y}$ type & HOMO & $p_{z}$ type & $p_{x} p_{y}$ type \\
\hline 1 & 28 & 27 & 26 & 28 & 26 & 27 \\
\hline 2 & 20 & 19 & 18 & 20 & 18 & 19 \\
\hline 3 & 24 & 23 & 22 & 24 & 22 & 23 \\
\hline 4 & 16 & 15 & 14 & 16 & 14 & 15 \\
\hline 5 & 36 & 33 & 32 & 36 & 33 & 36 \\
\hline 6 & 23 & 21 & 20 & 23 & 21 & 22 \\
\hline 7 & 36 & 33 & 32 & 36 & 34 & 35 \\
\hline 8 & 36 & 33 & 32 & 36 & 34 & 36 \\
\hline 9 & 31 & 30 & 29 & 31 & 29 & 30 \\
\hline 10 & 32 & 29 & 28 & 32 & 30 & 32 \\
\hline 11 & 36 & 33 & 32 & 36 & 33 & 36 \\
\hline 12 & 28 & 27 & 26 & 28 & 26 & 27 \\
\hline
\end{tabular}




\begin{tabular}{|c|c|c|c|c|c|c|}
\hline 13 & 40 & 37 & 36 & 40 & 37 & 40 \\
\hline 14 & 49 & 46 & 45 & 49 & 46 & 49 \\
\hline 15 & 36 & 33 & 32 & 36 & 33 & 36 \\
\hline 16 & 40 & 37 & 36 & 40 & 38 & 40 \\
\hline 17 & 20 & 19 & 18 & 20 & 18 & 19 \\
\hline 18 & 49 & 46 & 45 & 49 & 46 & 49 \\
\hline 19 & 12 & 11 & 10 & 12 & 10 & 11 \\
\hline 20 & 38 & 35 & 34 & 38 & 36 & 38 \\
\hline 21 & 38 & 35 & 34 & 38 & 36 & 38 \\
\hline 22 & 24 & 23 & 22 & 24 & 22 & 23 \\
\hline 23 & 22 & 20 & 18 & 22 & 20 & 21 \\
\hline 24 & 30 & 29 & 27 & 30 & 28 & 30 \\
\hline 25 & 33 & 31 & 29 & 33 & 31 & 32 \\
\hline 26 & 27 & 26 & 25 & 27 & 25 & 26 \\
\hline 27 & 24 & 23 & 22 & 24 & 22 & 23 \\
\hline 28 & 28 & 27 & 26 & 28 & 26 & 27 \\
\hline 29 & 20 & 19 & 18 & 20 & 18 & 19 \\
\hline 30 & 22 & 21 & 20 & 22 & 20 & 21 \\
\hline 31 & 27 & 26 & 25 & 27 & 25 & 26 \\
\hline 32 & 32 & 31 & 30 & 32 & 30 & 31 \\
\hline 33 & 23 & 22 & 21 & 23 & 21 & 22 \\
\hline 34 & 24 & 23 & 22 & 24 & 22 & 23 \\
\hline 35 & 40 & 39 & 38 & 40 & 38 & 39 \\
\hline 36 & 28 & 28 & 26 & 28 & 26 & 27 \\
\hline 37 & 21 & 21 & 20 & 21 & 20 & 21 \\
\hline 38 & 17 & 17 & 16 & 17 & 17 & 16 \\
\hline 39 & 17 & 17 & 16 & 17 & 17 & 16 \\
\hline 40 & 13 & 13 & 12 & 13 & 13 & 12 \\
\hline 41 & 9 & 9 & 8 & 9 & 8 & 9 \\
\hline 42 & 25 & 25 & 24 & 25 & 25 & 24 \\
\hline 43 & 29 & 29 & 27 & 29 & 29 & 28 \\
\hline 44 & 33 & 33 & 31 & 33 & 33 & 32 \\
\hline 45 & 29 & 29 & 27 & 29 & 29 & 28 \\
\hline 46 & 29 & 29 & 27 & 29 & 29 & 28 \\
\hline 47 & 25 & 25 & 23 & 25 & 25 & 24 \\
\hline 48 & 29 & 29 & 27 & 29 & 29 & 28 \\
\hline 49 & 29 & 29 & 27 & 29 & 29 & 28 \\
\hline 50 & 29 & 29 & 27 & 29 & 29 & 28 \\
\hline 51 & 33 & 33 & 31 & 33 & 33 & 31 \\
\hline 52 & 29 & 29 & 27 & 29 & 29 & 27 \\
\hline 53 & 33 & 33 & 31 & 33 & 33 & 32 \\
\hline
\end{tabular}




\begin{tabular}{lllllll}
$\mathbf{5 4}$ & 42 & 42 & 40 & 42 & 42 & 41 \\
$\mathbf{5 5}$ & 29 & 29 & 27 & 29 & 29 & 28 \\
$\mathbf{5 6}$ & 42 & 42 & 40 & 42 & 42 & 41 \\
$\mathbf{5 7}$ & 33 & 33 & 31 & 33 & 33 & 32 \\
$\mathbf{5 8}$ & 31 & 31 & 29 & 31 & 31 & 30 \\
$\mathbf{5 9}$ & 36 & 36 & 34 & 36 & 36 & 35 \\
$\mathbf{6 0}$ & 31 & 31 & 29 & 31 & 31 & 30 \\
$\mathbf{6 1}$ & 36 & 36 & 34 & 36 & 36 & 35 \\
\hline
\end{tabular}

S2E) COSMO/6-31+G(d,p)

\begin{tabular}{|c|c|c|c|c|c|c|}
\hline \multirow{2}{*}{ Compound } & \multicolumn{3}{|c|}{ Hartree-Fock } & \multicolumn{3}{|c|}{ DFT/B3LYP } \\
\hline & HOMO & $p_{z}$ type & $p_{x} p_{y}$ type & HOMO & $p_{z}$ type & $p_{x} p_{y}$ type \\
\hline 1 & 28 & 27 & 26 & 28 & 26 & 27 \\
\hline 2 & 20 & 19 & 18 & 20 & 18 & 19 \\
\hline 3 & 24 & 23 & 22 & 24 & 22 & 23 \\
\hline 4 & 16 & 15 & 14 & 16 & 14 & 15 \\
\hline 5 & 36 & 33 & 32 & 36 & 32 & 33 \\
\hline 6 & 23 & 21 & 20 & 23 & 21 & 22 \\
\hline 7 & 36 & 33 & 32 & 36 & 32 & 35 \\
\hline 8 & 36 & 33 & 32 & 36 & 32 & 34 \\
\hline 9 & 31 & 30 & 29 & 31 & 29 & 30 \\
\hline 10 & 32 & 29 & 28 & 32 & 28 & 31 \\
\hline 11 & 36 & 33 & 32 & 36 & 32 & 35 \\
\hline 12 & 28 & 27 & 26 & 28 & 26 & 27 \\
\hline 13 & 40 & 37 & 36 & 40 & 36 & 39 \\
\hline 14 & 49 & 46 & 44 & 49 & 45 & 48 \\
\hline 15 & 36 & 33 & 32 & 36 & 32 & 35 \\
\hline 16 & 40 & 37 & 36 & 40 & 36 & 38 \\
\hline 17 & 20 & 19 & 18 & 20 & 18 & 19 \\
\hline 18 & 49 & 45 & 43 & 49 & 45 & 47 \\
\hline 19 & 12 & 11 & 10 & 12 & 10 & 11 \\
\hline 20 & 38 & 35 & 34 & 38 & 34 & 38 \\
\hline 21 & 38 & 35 & 34 & 38 & 34 & 38 \\
\hline 22 & 24 & 23 & 21 & 24 & 21 & 22 \\
\hline 23 & 22 & 20 & 18 & 22 & 19 & 21 \\
\hline 24 & 30 & 29 & 27 & 30 & 28 & 30 \\
\hline 25 & 33 & 30 & 29 & 33 & 30 & 32 \\
\hline 26 & 27 & 26 & 25 & 27 & 25 & 26 \\
\hline 27 & 24 & 23 & 22 & 24 & 22 & 23 \\
\hline 28 & 28 & 27 & 25 & 28 & 26 & 27 \\
\hline 29 & 20 & 19 & 18 & 20 & 18 & 19 \\
\hline
\end{tabular}




\begin{tabular}{|c|c|c|c|c|c|c|}
\hline 30 & 22 & 21 & 20 & 22 & 20 & 21 \\
\hline 31 & 27 & 26 & 25 & 27 & 25 & 26 \\
\hline 32 & 32 & 31 & 29 & 32 & 30 & 31 \\
\hline 33 & 23 & 22 & 21 & 23 & 21 & 22 \\
\hline 34 & 24 & 23 & 22 & 24 & 22 & 23 \\
\hline 35 & 40 & 39 & 38 & 40 & 38 & 39 \\
\hline 36 & 28 & 28 & 26 & 28 & 26 & 27 \\
\hline 37 & 21 & 21 & 20 & 21 & 20 & 21 \\
\hline 38 & 17 & 17 & 16 & 17 & 17 & 16 \\
\hline 39 & 17 & 17 & 16 & 17 & 17 & 16 \\
\hline 40 & 13 & 13 & 12 & 13 & 13 & 12 \\
\hline 41 & 9 & 9 & 8 & 9 & 8 & 9 \\
\hline 42 & 25 & 25 & 24 & 25 & 25 & 24 \\
\hline 43 & 29 & 29 & 27 & 29 & 29 & 28 \\
\hline 44 & 33 & 33 & 31 & 33 & 33 & 32 \\
\hline 45 & 29 & 29 & 27 & 29 & 29 & 28 \\
\hline 46 & 29 & 29 & 27 & 29 & 29 & 28 \\
\hline 47 & 25 & 25 & 23 & 25 & 25 & 24 \\
\hline 48 & 29 & 29 & 27 & 29 & 29 & 28 \\
\hline 49 & 29 & 29 & 27 & 29 & 29 & 28 \\
\hline 50 & 29 & 29 & 27 & 29 & 29 & 28 \\
\hline 51 & 33 & 33 & 31 & 33 & 33 & 31 \\
\hline 52 & 29 & 29 & 27 & 29 & 29 & 27 \\
\hline 53 & 33 & 33 & 31 & 33 & 33 & 32 \\
\hline 54 & 42 & 42 & 40 & 42 & 42 & 41 \\
\hline 55 & 29 & 29 & 27 & 29 & 29 & 28 \\
\hline 56 & 42 & 42 & 40 & 42 & 42 & 41 \\
\hline 57 & 33 & 33 & 31 & 33 & 33 & 32 \\
\hline 58 & 31 & 31 & 29 & 31 & 31 & 30 \\
\hline 59 & 36 & 36 & 34 & 36 & 36 & 35 \\
\hline 60 & 31 & 31 & 29 & 31 & 31 & 30 \\
\hline 61 & 36 & 36 & 34 & 36 & 36 & 35 \\
\hline
\end{tabular}

Part S3. Molecular orbital energies (in hartrees)

S3A) 6-31G(d,p)

\begin{tabular}{ccccccc}
\hline \multirow{2}{*}{ Compound } & \multicolumn{3}{c}{ Hartree-Fock } & \multicolumn{3}{c}{ DFT/B3LYP } \\
& HOMO & $\boldsymbol{p}_{\boldsymbol{z}}$ type & $\boldsymbol{p}_{x} \boldsymbol{p}_{y}$ type & HOMO & $\boldsymbol{p}_{\boldsymbol{z}}$ type & $\boldsymbol{p}_{\boldsymbol{x}} \boldsymbol{p}_{\boldsymbol{y}}$ type \\
\hline $\mathbf{1}$ & -0.171 & -0.175 & -0.191 & -0.011 & -0.028 & -0.011 \\
$\mathbf{2}$ & -0.166 & -0.166 & -0.182 & 0.002 & -0.016 & 0.002 \\
$\mathbf{3}$ & -0.170 & -0.170 & -0.187 & -0.004 & -0.021 & -0.004
\end{tabular}




\begin{tabular}{|c|c|c|c|c|c|c|}
\hline 4 & -0.160 & -0.160 & -0.176 & 0.009 & -0.008 & 0.009 \\
\hline 5 & -0.177 & -0.183 & -0.203 & -0.024 & -0.038 & -0.024 \\
\hline 6 & -0.167 & -0.178 & -0.195 & -0.013 & -0.030 & -0.013 \\
\hline 7 & -0.165 & -0.185 & -0.198 & -0.024 & -0.042 & -0.024 \\
\hline 8 & -0.180 & -0.180 & -0.204 & -0.025 & -0.038 & -0.025 \\
\hline 9 & -0.186 & -0.186 & -0.203 & -0.020 & -0.038 & -0.020 \\
\hline 10 & -0.183 & -0.183 & -0.204 & -0.025 & -0.038 & -0.025 \\
\hline 11 & -0.190 & -0.190 & -0.210 & -0.030 & -0.043 & -0.030 \\
\hline 12 & -0.192 & -0.192 & -0.210 & -0.029 & -0.044 & -0.029 \\
\hline 13 & -0.195 & -0.195 & -0.216 & -0.037 & -0.050 & -0.037 \\
\hline 14 & -0.195 & -0.195 & -0.214 & -0.038 & -0.051 & -0.038 \\
\hline 15 & -0.191 & -0.191 & -0.213 & -0.032 & -0.045 & -0.032 \\
\hline 16 & -0.196 & -0.196 & -0.217 & -0.039 & -0.051 & -0.039 \\
\hline 17 & -0.167 & -0.167 & -0.186 & -0.002 & -0.015 & -0.002 \\
\hline 18 & -0.196 & -0.196 & -0.218 & -0.040 & -0.053 & -0.040 \\
\hline 19 & -0.158 & -0.160 & -0.181 & 0.011 & -0.006 & 0.011 \\
\hline 20 & -0.204 & -0.204 & -0.225 & -0.047 & -0.059 & -0.047 \\
\hline 21 & -0.206 & -0.206 & -0.228 & -0.049 & -0.061 & -0.049 \\
\hline 22 & -0.171 & -0.171 & -0.191 & -0.008 & -0.021 & -0.008 \\
\hline 23 & -0.174 & -0.183 & -0.201 & -0.019 & -0.034 & -0.019 \\
\hline 24 & -0.194 & -0.194 & -0.216 & -0.032 & -0.046 & -0.032 \\
\hline 25 & -0.197 & -0.198 & -0.215 & -0.035 & -0.049 & -0.035 \\
\hline 26 & -0.191 & -0.195 & -0.214 & -0.031 & -0.044 & -0.031 \\
\hline 27 & -0.194 & -0.194 & -0.212 & -0.031 & -0.044 & -0.031 \\
\hline 28 & -0.191 & -0.191 & -0.212 & -0.028 & -0.042 & -0.028 \\
\hline 29 & -0.178 & -0.178 & -0.199 & -0.012 & -0.025 & -0.012 \\
\hline 30 & -0.201 & -0.201 & -0.223 & -0.039 & -0.053 & -0.039 \\
\hline 31 & -0.214 & -0.214 & -0.238 & -0.053 & -0.066 & -0.053 \\
\hline 32 & -0.211 & -0.211 & -0.233 & -0.050 & -0.063 & -0.050 \\
\hline 33 & -0.194 & -0.194 & -0.218 & -0.026 & -0.041 & -0.026 \\
\hline 34 & -0.191 & -0.191 & -0.215 & -0.024 & -0.037 & -0.024 \\
\hline 35 & -0.227 & -0.231 & -0.248 & -0.070 & -0.082 & -0.070 \\
\hline 36 & -0.205 & -0.205 & -0.231 & -0.037 & -0.048 & -0.037 \\
\hline 37 & -0.095 & -0.095 & -0.083 & 0.054 & 0.054 & 0.054 \\
\hline 38 & -0.087 & -0.087 & -0.091 & 0.060 & 0.060 & 0.059 \\
\hline 39 & -0.083 & -0.083 & -0.086 & 0.065 & 0.065 & 0.062 \\
\hline 40 & -0.077 & -0.077 & -0.082 & 0.072 & 0.072 & 0.067 \\
\hline 41 & -0.071 & -0.071 & -0.071 & 0.081 & 0.081 & 0.081 \\
\hline 42 & -0.114 & -0.114 & -0.126 & 0.042 & 0.042 & 0.031 \\
\hline 43 & -0.064 & -0.064 & -0.173 & 0.019 & 0.019 & -0.009 \\
\hline 44 & -0.059 & -0.059 & -0.172 & 0.024 & 0.024 & -0.008 \\
\hline
\end{tabular}




\begin{tabular}{lllllll}
$\mathbf{4 5}$ & -0.062 & -0.062 & -0.173 & 0.020 & 0.020 & -0.009 \\
$\mathbf{4 6}$ & -0.066 & -0.066 & -0.172 & 0.018 & 0.018 & -0.008 \\
$\mathbf{4 7}$ & -0.065 & -0.065 & -0.172 & 0.020 & 0.020 & -0.007 \\
$\mathbf{4 8}$ & -0.056 & -0.056 & -0.170 & 0.028 & 0.028 & -0.006 \\
$\mathbf{4 9}$ & -0.069 & -0.069 & -0.178 & 0.017 & 0.017 & -0.013 \\
$\mathbf{5 0}$ & -0.068 & -0.068 & -0.175 & 0.016 & 0.016 & -0.010 \\
$\mathbf{5 1}$ & -0.073 & -0.073 & -0.178 & 0.014 & 0.014 & -0.012 \\
$\mathbf{5 2}$ & -0.073 & -0.073 & -0.180 & 0.015 & 0.015 & -0.013 \\
$\mathbf{5 3}$ & -0.082 & -0.082 & -0.191 & 0.003 & 0.003 & -0.025 \\
$\mathbf{5 4}$ & -0.085 & -0.085 & -0.194 & 0.000 & 0.000 & -0.027 \\
$\mathbf{5 5}$ & -0.080 & -0.080 & -0.187 & 0.008 & 0.008 & -0.018 \\
$\mathbf{5 6}$ & -0.088 & -0.088 & -0.194 & -0.004 & -0.004 & -0.031 \\
$\mathbf{5 7}$ & -0.086 & -0.086 & -0.193 & -0.002 & -0.002 & -0.029 \\
$\mathbf{5 8}$ & -0.096 & -0.096 & -0.203 & -0.013 & -0.013 & -0.038 \\
$\mathbf{5 9}$ & -0.098 & -0.098 & -0.207 & -0.017 & -0.017 & -0.041 \\
$\mathbf{6 0}$ & -0.103 & -0.103 & -0.215 & -0.023 & -0.023 & -0.049 \\
$\mathbf{6 1}$ & -0.120 & -0.120 & -0.231 & -0.039 & -0.039 & -0.064 \\
\hline
\end{tabular}

S3B) PCM/6-31G(d,p)

\begin{tabular}{ccccccc}
\hline Compound & \multicolumn{3}{c}{ Hartree-Fock } & & \multicolumn{3}{c}{ DFT/B3LYP } \\
& HOMO & $\boldsymbol{p}_{\boldsymbol{z}}$ type & $\boldsymbol{p}_{\boldsymbol{x}} \boldsymbol{p}_{\boldsymbol{y}}$ type & HOMO & $\boldsymbol{p}_{\boldsymbol{z}}$ type & $\boldsymbol{p}_{\boldsymbol{x}} \boldsymbol{p}_{\boldsymbol{y}}$ type \\
\hline $\mathbf{1}$ & -0.363 & -0.389 & -0.399 & -0.205 & -0.232 & -0.214 \\
$\mathbf{2}$ & -0.374 & -0.391 & -0.401 & -0.209 & -0.232 & -0.214 \\
$\mathbf{3}$ & -0.373 & -0.390 & -0.400 & -0.209 & -0.232 & -0.214 \\
$\mathbf{4}$ & -0.379 & -0.391 & -0.401 & -0.210 & -0.231 & -0.212 \\
$\mathbf{5}$ & -0.318 & -0.401 & -0.412 & -0.226 & -0.238 & -0.226 \\
$\mathbf{6}$ & -0.335 & -0.397 & -0.410 & -0.205 & -0.238 & -0.221 \\
$\mathbf{7}$ & -0.309 & -0.399 & -0.408 & -0.206 & -0.238 & -0.221 \\
$\mathbf{8}$ & -0.318 & -0.400 & -0.411 & -0.225 & -0.238 & -0.225 \\
$\mathbf{9}$ & -0.380 & -0.396 & -0.406 & -0.215 & -0.239 & -0.222 \\
$\mathbf{1 0}$ & -0.329 & -0.401 & -0.413 & -0.227 & -0.240 & -0.227 \\
$\mathbf{1 1}$ & -0.332 & -0.401 & -0.413 & -0.227 & -0.241 & -0.227 \\
$\mathbf{1 2}$ & -0.379 & -0.397 & -0.410 & -0.217 & -0.240 & -0.225 \\
$\mathbf{1 3}$ & -0.331 & -0.403 & -0.415 & -0.229 & -0.244 & -0.229 \\
$\mathbf{1 4}$ & -0.330 & -0.402 & -0.412 & -0.229 & -0.243 & -0.229 \\
$\mathbf{1 5}$ & -0.331 & -0.401 & -0.414 & -0.227 & -0.244 & -0.227 \\
$\mathbf{1 6}$ & -0.331 & -0.404 & -0.416 & -0.230 & -0.247 & -0.230 \\
$\mathbf{1 7}$ & -0.381 & -0.393 & -0.410 & -0.214 & -0.233 & -0.222 \\
$\mathbf{1 8}$ & -0.329 & -0.404 & -0.414 & -0.231 & -0.246 & -0.231 \\
$\mathbf{1 9}$ & -0.381 & -0.388 & -0.410 & -0.210 & -0.231 & -0.220 \\
$\mathbf{2 0}$ & -0.342 & -0.404 & -0.418 & -0.232 & -0.246 & -0.232 \\
& & & & & &
\end{tabular}




\begin{tabular}{|c|c|c|c|c|c|c|}
\hline 21 & -0.341 & -0.402 & -0.416 & -0.229 & -0.244 & -0.229 \\
\hline 22 & -0.386 & -0.397 & -0.415 & -0.219 & -0.236 & -0.227 \\
\hline 23 & -0.354 & -0.402 & -0.420 & -0.213 & -0.241 & -0.226 \\
\hline 24 & -0.381 & -0.400 & -0.416 & -0.227 & -0.243 & -0.227 \\
\hline 25 & -0.377 & -0.407 & -0.415 & -0.225 & -0.246 & -0.232 \\
\hline 26 & -0.383 & -0.406 & -0.419 & -0.222 & -0.247 & -0.232 \\
\hline 27 & -0.389 & -0.404 & -0.418 & -0.226 & -0.246 & -0.232 \\
\hline 28 & -0.385 & -0.402 & -0.417 & -0.223 & -0.246 & -0.230 \\
\hline 29 & -0.391 & -0.400 & -0.418 & -0.219 & -0.240 & -0.228 \\
\hline 30 & -0.386 & -0.408 & -0.423 & -0.227 & -0.250 & -0.236 \\
\hline 31 & -0.396 & -0.416 & -0.434 & -0.235 & -0.259 & -0.247 \\
\hline 32 & -0.396 & -0.412 & -0.430 & -0.236 & -0.256 & -0.243 \\
\hline 33 & -0.400 & -0.409 & -0.430 & -0.225 & -0.251 & -0.238 \\
\hline 34 & -0.404 & -0.407 & -0.428 & -0.228 & -0.249 & -0.236 \\
\hline 35 & -0.399 & -0.423 & -0.429 & -0.247 & -0.266 & -0.251 \\
\hline 36 & -0.414 & -0.414 & -0.438 & -0.240 & -0.254 & -0.243 \\
\hline 37 & -0.322 & -0.322 & -0.322 & -0.161 & -0.162 & -0.161 \\
\hline 38 & -0.325 & -0.325 & -0.328 & -0.168 & -0.168 & -0.168 \\
\hline 39 & -0.335 & -0.335 & -0.338 & -0.176 & -0.176 & -0.179 \\
\hline 40 & -0.329 & -0.329 & -0.331 & -0.169 & -0.169 & -0.171 \\
\hline 41 & -0.334 & -0.334 & -0.334 & -0.171 & -0.171 & -0.171 \\
\hline 42 & -0.352 & -0.352 & -0.368 & -0.186 & -0.186 & -0.197 \\
\hline 43 & -0.241 & -0.241 & -0.370 & -0.154 & -0.154 & -0.202 \\
\hline 44 & -0.239 & -0.239 & -0.374 & -0.150 & -0.150 & -0.205 \\
\hline 45 & -0.239 & -0.239 & -0.370 & -0.153 & -0.153 & -0.202 \\
\hline 46 & -0.248 & -0.248 & -0.373 & -0.160 & -0.160 & -0.205 \\
\hline 47 & -0.246 & -0.246 & -0.372 & -0.158 & -0.158 & -0.204 \\
\hline 48 & -0.236 & -0.236 & -0.372 & -0.147 & -0.147 & -0.203 \\
\hline 49 & -0.249 & -0.249 & -0.376 & -0.158 & -0.158 & -0.206 \\
\hline 50 & -0.247 & -0.247 & -0.370 & -0.159 & -0.159 & -0.202 \\
\hline 51 & -0.254 & -0.254 & -0.379 & -0.162 & -0.162 & -0.207 \\
\hline 52 & -0.252 & -0.252 & -0.378 & -0.160 & -0.160 & -0.207 \\
\hline 53 & -0.254 & -0.254 & -0.380 & -0.164 & -0.164 & -0.210 \\
\hline 54 & -0.253 & -0.253 & -0.381 & -0.164 & -0.164 & -0.210 \\
\hline 55 & -0.257 & -0.257 & -0.384 & -0.166 & -0.166 & -0.211 \\
\hline 56 & -0.258 & -0.258 & -0.380 & -0.170 & -0.170 & -0.213 \\
\hline 57 & -0.258 & -0.258 & -0.383 & -0.169 & -0.169 & -0.214 \\
\hline 58 & -0.261 & -0.261 & -0.386 & -0.175 & -0.175 & -0.216 \\
\hline 59 & -0.262 & -0.262 & -0.388 & -0.176 & -0.176 & -0.217 \\
\hline 60 & -0.264 & -0.264 & -0.389 & -0.179 & -0.179 & -0.220 \\
\hline 61 & -0.277 & -0.277 & -0.397 & -0.190 & -0.190 & -0.227 \\
\hline
\end{tabular}


S3C) $P C M / 6-31+G(d, p)$

\begin{tabular}{|c|c|c|c|c|c|c|}
\hline \multirow{2}{*}{ Compound } & \multicolumn{3}{|c|}{ Hartree-Fock } & \multicolumn{3}{|c|}{ DFT/B3LYP } \\
\hline & HOMO & $p_{z}$ type & $p_{x} p_{y}$ type & HOMO & $p_{z}$ type & $p_{x} p_{y}$ type \\
\hline 1 & -0.383 & -0.413 & -0.423 & -0.240 & -0.271 & -0.252 \\
\hline 2 & -0.396 & -0.418 & -0.426 & -0.247 & -0.274 & -0.255 \\
\hline 3 & -0.394 & -0.415 & -0.424 & -0.247 & -0.273 & -0.254 \\
\hline 4 & -0.404 & -0.420 & -0.428 & -0.252 & -0.277 & -0.257 \\
\hline 5 & -0.329 & -0.425 & -0.436 & -0.250 & -0.281 & -0.262 \\
\hline 6 & -0.348 & -0.423 & -0.435 & -0.238 & -0.281 & -0.261 \\
\hline 7 & -0.321 & -0.423 & -0.431 & -0.233 & -0.280 & -0.253 \\
\hline 8 & -0.328 & -0.424 & -0.435 & -0.249 & -0.280 & -0.261 \\
\hline 9 & -0.401 & -0.421 & -0.430 & -0.251 & -0.278 & -0.259 \\
\hline 10 & -0.340 & -0.425 & -0.437 & -0.260 & -0.282 & -0.263 \\
\hline 11 & -0.346 & -0.425 & -0.437 & -0.259 & -0.282 & -0.263 \\
\hline 12 & -0.399 & -0.421 & -0.434 & -0.253 & -0.279 & -0.263 \\
\hline 13 & -0.342 & -0.426 & -0.438 & -0.256 & -0.283 & -0.264 \\
\hline 14 & -0.341 & -0.425 & -0.439 & -0.255 & -0.283 & -0.263 \\
\hline 15 & -0.343 & -0.425 & -0.438 & -0.257 & -0.282 & -0.263 \\
\hline 16 & -0.341 & -0.426 & -0.439 & -0.256 & -0.283 & -0.266 \\
\hline 17 & -0.406 & -0.419 & -0.436 & -0.255 & -0.272 & -0.264 \\
\hline 18 & -0.339 & -0.427 & -0.440 & -0.254 & -0.283 & -0.266 \\
\hline 19 & -0.408 & -0.419 & -0.438 & -0.253 & -0.278 & -0.267 \\
\hline 20 & -0.354 & -0.427 & -0.441 & -0.266 & -0.285 & -0.267 \\
\hline 21 & -0.353 & -0.426 & -0.440 & -0.265 & -0.285 & -0.265 \\
\hline 22 & -0.409 & -0.419 & -0.440 & -0.258 & -0.285 & -0.268 \\
\hline 23 & -0.372 & -0.430 & -0.445 & -0.248 & -0.286 & -0.267 \\
\hline 24 & -0.395 & -0.425 & -0.441 & -0.265 & -0.282 & -0.265 \\
\hline 25 & -0.393 & -0.432 & -0.438 & -0.259 & -0.290 & -0.270 \\
\hline 26 & -0.405 & -0.430 & -0.441 & -0.258 & -0.286 & -0.270 \\
\hline 27 & -0.408 & -0.425 & -0.441 & -0.261 & -0.284 & -0.270 \\
\hline 28 & -0.403 & -0.425 & -0.444 & -0.257 & -0.283 & -0.268 \\
\hline 29 & -0.416 & -0.428 & -0.444 & -0.261 & -0.283 & -0.271 \\
\hline 30 & -0.407 & -0.432 & -0.447 & -0.263 & -0.288 & -0.274 \\
\hline 31 & -0.417 & -0.439 & -0.457 & -0.270 & -0.295 & -0.284 \\
\hline 32 & -0.413 & -0.434 & -0.449 & -0.267 & -0.292 & -0.279 \\
\hline 33 & -0.422 & -0.433 & -0.454 & -0.262 & -0.290 & -0.278 \\
\hline 34 & -0.430 & -0.433 & -0.454 & -0.270 & -0.290 & -0.279 \\
\hline 35 & -0.413 & -0.441 & -0.442 & -0.273 & -0.298 & -0.280 \\
\hline 36 & -0.439 & -0.439 & -0.463 & -0.283 & -0.296 & -0.285 \\
\hline 37 & -0.349 & -0.349 & -0.350 & -0.211 & -0.212 & -0.211 \\
\hline 38 & -0.352 & -0.352 & -0.355 & -0.214 & -0.214 & -0.215 \\
\hline
\end{tabular}




\begin{tabular}{|c|c|c|c|c|c|c|}
\hline 39 & -0.361 & -0.361 & -0.365 & -0.221 & -0.221 & -0.224 \\
\hline 40 & -0.356 & -0.356 & -0.358 & -0.215 & -0.215 & -0.217 \\
\hline 41 & -0.362 & -0.362 & -0.362 & -0.217 & -0.217 & -0.217 \\
\hline 42 & -0.378 & -0.378 & -0.396 & -0.229 & -0.229 & -0.242 \\
\hline 43 & -0.259 & -0.259 & -0.393 & -0.181 & -0.181 & -0.239 \\
\hline 44 & -0.256 & -0.256 & -0.396 & -0.176 & -0.176 & -0.240 \\
\hline 45 & -0.256 & -0.256 & -0.393 & -0.179 & -0.179 & -0.238 \\
\hline 46 & -0.265 & -0.265 & -0.395 & -0.186 & -0.186 & -0.241 \\
\hline 47 & -0.265 & -0.265 & -0.396 & -0.186 & -0.186 & -0.241 \\
\hline 48 & -0.254 & -0.254 & -0.395 & -0.174 & -0.174 & -0.239 \\
\hline 49 & -0.268 & -0.268 & -0.400 & -0.186 & -0.186 & -0.242 \\
\hline 50 & -0.265 & -0.265 & -0.393 & -0.186 & -0.186 & -0.238 \\
\hline 51 & -0.271 & -0.271 & -0.402 & -0.189 & -0.189 & -0.243 \\
\hline 52 & -0.269 & -0.269 & -0.402 & -0.187 & -0.187 & -0.243 \\
\hline 53 & -0.270 & -0.270 & -0.402 & -0.189 & -0.189 & -0.245 \\
\hline 54 & -0.270 & -0.270 & -0.402 & -0.189 & -0.189 & -0.245 \\
\hline 55 & -0.275 & -0.275 & -0.407 & -0.193 & -0.193 & -0.247 \\
\hline 56 & -0.275 & -0.275 & -0.401 & -0.196 & -0.196 & -0.245 \\
\hline 57 & -0.274 & -0.274 & -0.404 & -0.195 & -0.195 & -0.246 \\
\hline 58 & -0.278 & -0.278 & -0.408 & -0.200 & -0.200 & -0.250 \\
\hline 59 & -0.278 & -0.278 & -0.409 & -0.201 & -0.201 & -0.250 \\
\hline 60 & -0.280 & -0.280 & -0.409 & -0.203 & -0.203 & -0.252 \\
\hline 61 & -0.292 & -0.292 & -0.415 & -0.214 & -0.214 & -0.257 \\
\hline \multicolumn{7}{|c|}{ S3D) COSMO/6-31G(d,p) } \\
\hline \multirow{2}{*}{ Compound } & \multicolumn{3}{|c|}{ Hartree-Fock } & \multicolumn{3}{|c|}{ DFT/B3LYP } \\
\hline & HOMO & $p_{z}$ type & $p_{x} p_{y}$ type & HOMO & $p_{z}$ type & $p_{x} p_{y}$ type \\
\hline 1 & -0.362 & -0.387 & -0.397 & -0.204 & -0.230 & -0.212 \\
\hline 2 & -0.373 & -0.389 & -0.399 & -0.208 & -0.231 & -0.212 \\
\hline 3 & -0.371 & -0.386 & -0.398 & -0.207 & -0.229 & -0.211 \\
\hline 4 & -0.378 & -0.389 & -0.400 & -0.210 & -0.230 & -0.211 \\
\hline 5 & -0.318 & -0.397 & -0.409 & -0.223 & -0.236 & -0.223 \\
\hline 6 & -0.335 & -0.395 & -0.408 & -0.204 & -0.236 & -0.219 \\
\hline 7 & -0.310 & -0.395 & -0.405 & -0.204 & -0.236 & -0.218 \\
\hline 8 & -0.318 & -0.397 & -0.409 & -0.223 & -0.231 & -0.223 \\
\hline 9 & -0.379 & -0.395 & -0.405 & -0.214 & -0.237 & -0.220 \\
\hline 10 & -0.328 & -0.398 & -0.410 & -0.224 & -0.237 & -0.224 \\
\hline 11 & -0.331 & -0.398 & -0.410 & -0.224 & -0.239 & -0.224 \\
\hline 12 & -0.378 & -0.395 & -0.409 & -0.216 & -0.238 & -0.223 \\
\hline 13 & -0.331 & -0.399 & -0.412 & -0.226 & -0.240 & -0.226 \\
\hline 14 & -0.328 & -0.399 & -0.410 & -0.226 & -0.240 & -0.226 \\
\hline
\end{tabular}




\begin{tabular}{|c|c|c|c|c|c|c|}
\hline 15 & -0.331 & -0.399 & -0.412 & -0.225 & -0.243 & -0.225 \\
\hline 16 & -0.332 & -0.400 & -0.413 & -0.227 & -0.239 & -0.227 \\
\hline 17 & -0.383 & -0.394 & -0.411 & -0.215 & -0.234 & -0.222 \\
\hline 18 & -0.329 & -0.400 & -0.413 & -0.227 & -0.244 & -0.227 \\
\hline 19 & -0.381 & -0.388 & -0.409 & -0.210 & -0.231 & -0.220 \\
\hline 20 & -0.343 & -0.402 & -0.415 & -0.230 & -0.244 & -0.230 \\
\hline 21 & -0.342 & -0.400 & -0.414 & -0.228 & -0.242 & -0.228 \\
\hline 22 & -0.384 & -0.394 & -0.412 & -0.217 & -0.234 & -0.224 \\
\hline 23 & -0.355 & -0.400 & -0.419 & -0.212 & -0.240 & -0.225 \\
\hline 24 & -0.382 & -0.400 & -0.416 & -0.227 & -0.242 & -0.227 \\
\hline 25 & -0.377 & -0.406 & -0.414 & -0.225 & -0.245 & -0.232 \\
\hline 26 & -0.382 & -0.403 & -0.416 & -0.220 & -0.245 & -0.229 \\
\hline 27 & -0.389 & -0.402 & -0.417 & -0.226 & -0.245 & -0.232 \\
\hline 28 & -0.383 & -0.399 & -0.415 & -0.221 & -0.243 & -0.228 \\
\hline 29 & -0.391 & -0.399 & -0.417 & -0.219 & -0.239 & -0.227 \\
\hline 30 & -0.386 & -0.407 & -0.423 & -0.227 & -0.249 & -0.235 \\
\hline 31 & -0.394 & -0.413 & -0.431 & -0.232 & -0.255 & -0.244 \\
\hline 32 & -0.393 & -0.409 & -0.427 & -0.233 & -0.254 & -0.241 \\
\hline 33 & -0.400 & -0.409 & -0.430 & -0.225 & -0.251 & -0.239 \\
\hline 34 & -0.403 & -0.406 & -0.427 & -0.227 & -0.247 & -0.235 \\
\hline 35 & -0.399 & -0.422 & -0.428 & -0.246 & -0.265 & -0.250 \\
\hline 36 & -0.415 & -0.415 & -0.439 & -0.241 & -0.255 & -0.244 \\
\hline 37 & -0.318 & -0.318 & -0.319 & -0.158 & -0.158 & -0.158 \\
\hline 38 & -0.322 & -0.322 & -0.325 & -0.163 & -0.163 & -0.164 \\
\hline 39 & -0.327 & -0.327 & -0.328 & -0.168 & -0.168 & -0.168 \\
\hline 40 & -0.326 & -0.326 & -0.328 & -0.165 & -0.165 & -0.168 \\
\hline 41 & -0.331 & -0.331 & -0.331 & -0.168 & -0.168 & -0.168 \\
\hline 42 & -0.347 & -0.347 & -0.363 & -0.181 & -0.181 & -0.192 \\
\hline 43 & -0.240 & -0.240 & -0.368 & -0.153 & -0.153 & -0.200 \\
\hline 44 & -0.238 & -0.238 & -0.371 & -0.149 & -0.149 & -0.2016 \\
\hline 45 & -0.238 & -0.238 & -0.367 & -0.152 & -0.152 & -0.199 \\
\hline 46 & -0.246 & -0.246 & -0.370 & -0.158 & -0.158 & -0.201 \\
\hline 47 & -0.246 & -0.246 & -0.370 & -0.158 & -0.158 & -0.201 \\
\hline 48 & -0.236 & -0.236 & -0.370 & -0.146 & -0.146 & -0.201 \\
\hline 49 & -0.248 & -0.248 & -0.375 & -0.157 & -0.157 & -0.204 \\
\hline 50 & -0.247 & -0.247 & -0.368 & -0.157 & -0.157 & -0.200 \\
\hline 51 & -0.253 & -0.253 & -0.376 & -0.160 & -0.160 & -0.202 \\
\hline 52 & -0.251 & -0.251 & -0.376 & -0.159 & -0.159 & -0.203 \\
\hline 53 & -0.253 & -0.253 & -0.378 & -0.163 & -0.163 & -0.208 \\
\hline 54 & -0.252 & -0.252 & -0.379 & -0.163 & -0.163 & -0.208 \\
\hline 55 & -0.257 & -0.257 & -0.381 & -0.165 & -0.165 & -0.208 \\
\hline
\end{tabular}




\begin{tabular}{lllllll}
$\mathbf{5 6}$ & -0.257 & -0.257 & -0.378 & -0.168 & -0.168 & -0.210 \\
$\mathbf{5 7}$ & -0.257 & -0.257 & -0.380 & -0.168 & -0.168 & -0.210 \\
$\mathbf{5 8}$ & -0.261 & -0.261 & -0.384 & -0.174 & -0.174 & -0.214 \\
$\mathbf{5 9}$ & -0.259 & -0.259 & -0.384 & -0.173 & -0.173 & -0.213 \\
$\mathbf{6 0}$ & -0.263 & -0.263 & -0.388 & -0.178 & -0.178 & -0.218 \\
$\mathbf{6 1}$ & -0.277 & -0.277 & -0.397 & -0.190 & -0.190 & -0.227 \\
\hline
\end{tabular}

S3E) $\operatorname{cosMO} / 6-31+G(d, p)$

\begin{tabular}{|c|c|c|c|c|c|c|}
\hline \multirow{2}{*}{ Compound } & \multicolumn{3}{|c|}{ Hartree-Fock } & \multicolumn{3}{|c|}{ DFT/B3LYP } \\
\hline & HOMO & $p_{z}$ type & $p_{x} p_{y}$ type & HOMO & $p_{z}$ type & $p_{x} p_{y}$ type \\
\hline 1 & -0.381 & -0.411 & -0.421 & -0.238 & -0.268 & -0.249 \\
\hline 2 & -0.395 & -0.416 & -0.424 & -0.246 & -0.272 & -0.252 \\
\hline 3 & -0.392 & -0.412 & -0.421 & -0.244 & -0.269 & -0.250 \\
\hline 4 & -0.403 & -0.418 & -0.426 & -0.251 & -0.274 & -0.254 \\
\hline 5 & -0.329 & -0.421 & -0.432 & -0.249 & -0.277 & -0.258 \\
\hline 6 & -0.348 & -0.420 & -0.432 & -0.237 & -0.274 & -0.258 \\
\hline 7 & -0.322 & -0.420 & -0.428 & -0.232 & -0.277 & -0.252 \\
\hline 8 & -0.328 & -0.421 & -0.433 & -0.249 & -0.277 & -0.258 \\
\hline 9 & -0.401 & -0.420 & -0.429 & -0.251 & -0.278 & -0.259 \\
\hline 10 & -0.340 & -0.422 & -0.434 & -0.259 & -0.279 & -0.260 \\
\hline 11 & -0.344 & -0.422 & -0.434 & -0.257 & -0.279 & -0.260 \\
\hline 12 & -0.398 & -0.419 & -0.432 & -0.251 & -0.276 & -0.260 \\
\hline 13 & -0.341 & -0.422 & -0.435 & -0.255 & -0.280 & -0.260 \\
\hline 14 & -0.339 & -0.422 & -0.436 & -0.253 & -0.279 & -0.260 \\
\hline 15 & -0.343 & -0.422 & -0.435 & -0.257 & -0.279 & -0.261 \\
\hline 16 & -0.341 & -0.423 & -0.436 & -0.256 & -0.280 & -0.262 \\
\hline 17 & -0.407 & -0.420 & -0.437 & -0.255 & -0.273 & -0.264 \\
\hline 18 & -0.339 & -0.423 & -0.436 & -0.254 & -0.279 & -0.261 \\
\hline 19 & -0.408 & -0.418 & -0.438 & -0.253 & -0.277 & -0.266 \\
\hline 20 & -0.354 & -0.424 & -0.438 & -0.264 & -0.283 & -0.264 \\
\hline 21 & -0.354 & -0.423 & -0.437 & -0.262 & -0.283 & -0.262 \\
\hline 22 & -0.407 & -0.417 & -0.437 & -0.255 & -0.282 & -0.264 \\
\hline 23 & -0.372 & -0.425 & -0.442 & -0.246 & -0.283 & -0.264 \\
\hline 24 & -0.396 & -0.424 & -0.440 & -0.265 & -0.281 & -0.265 \\
\hline 25 & -0.393 & -0.430 & -0.436 & -0.258 & -0.288 & -0.267 \\
\hline 26 & -0.403 & -0.427 & -0.439 & -0.256 & -0.283 & -0.266 \\
\hline 27 & -0.407 & -0.424 & -0.439 & -0.260 & -0.282 & -0.268 \\
\hline 28 & -0.401 & -0.422 & -0.442 & -0.254 & -0.280 & -0.264 \\
\hline 29 & -0.415 & -0.426 & -0.442 & -0.260 & -0.282 & -0.269 \\
\hline 30 & -0.407 & -0.430 & -0.446 & -0.262 & -0.286 & -0.272 \\
\hline 31 & -0.414 & -0.435 & -0.453 & -0.266 & -0.291 & -0.279 \\
\hline
\end{tabular}




\begin{tabular}{|c|c|c|c|c|c|c|}
\hline 32 & -0.409 & -0.430 & -0.445 & -0.263 & -0.287 & -0.274 \\
\hline 33 & -0.422 & -0.433 & -0.453 & -0.262 & -0.290 & -0.277 \\
\hline 34 & -0.428 & -0.431 & -0.451 & -0.268 & -0.288 & -0.276 \\
\hline 35 & -0.412 & -0.439 & -0.441 & -0.271 & -0.295 & -0.277 \\
\hline 36 & -0.438 & -0.438 & -0.462 & -0.282 & -0.294 & -0.283 \\
\hline 37 & -0.345 & -0.345 & -0.345 & -0.204 & -0.204 & -0.204 \\
\hline 38 & -0.347 & -0.347 & -0.351 & -0.207 & -0.207 & -0.208 \\
\hline 39 & -0.351 & -0.351 & -0.353 & -0.209 & -0.209 & -0.210 \\
\hline 40 & -0.351 & -0.351 & -0.354 & -0.208 & -0.208 & -0.212 \\
\hline 41 & -0.358 & -0.358 & -0.358 & -0.213 & -0.213 & -0.213 \\
\hline 42 & -0.371 & -0.371 & -0.390 & -0.221 & -0.221 & -0.235 \\
\hline 43 & -0.258 & -0.258 & -0.390 & -0.180 & -0.180 & -0.234 \\
\hline 44 & -0.254 & -0.254 & -0.392 & -0.174 & -0.174 & -0.235 \\
\hline 45 & -0.255 & -0.255 & -0.389 & -0.177 & -0.177 & -0.233 \\
\hline 46 & -0.263 & -0.263 & -0.391 & -0.184 & -0.184 & -0.235 \\
\hline 47 & -0.264 & -0.264 & -0.392 & -0.185 & -0.185 & -0.236 \\
\hline 48 & -0.253 & -0.253 & -0.392 & -0.173 & -0.173 & -0.236 \\
\hline 49 & -0.267 & -0.267 & -0.396 & -0.184 & -0.184 & -0.238 \\
\hline 50 & -0.264 & -0.264 & -0.390 & -0.185 & -0.185 & -0.234 \\
\hline 51 & -0.269 & -0.269 & -0.398 & -0.187 & -0.187 & -0.238 \\
\hline 52 & -0.268 & -0.268 & -0.398 & -0.186 & -0.186 & -0.239 \\
\hline 53 & -0.268 & -0.268 & -0.398 & -0.187 & -0.187 & -0.240 \\
\hline 54 & -0.268 & -0.268 & -0.398 & -0.187 & -0.187 & -0.240 \\
\hline 55 & -0.273 & -0.273 & -0.403 & -0.191 & -0.191 & -0.243 \\
\hline 56 & -0.272 & -0.272 & -0.397 & -0.192 & -0.192 & -0.242 \\
\hline 57 & -0.273 & -0.273 & -0.400 & -0.192 & -0.192 & -0.242 \\
\hline 58 & -0.277 & -0.277 & -0.405 & -0.199 & -0.199 & -0.246 \\
\hline 59 & -0.274 & -0.274 & -0.404 & -0.197 & -0.197 & -0.245 \\
\hline 60 & -0.279 & -0.279 & -0.406 & -0.201 & -0.201 & -0.248 \\
\hline 61 & -0.292 & -0.292 & -0.414 & -0.214 & -0.214 & -0.255 \\
\hline
\end{tabular}

Part S4. Molecular Orbital compositions (\%)

S4A) Carboxylate ions - DFT/COSMO/6-31+G(d,p)

\section{Compound 1:}

\begin{tabular}{lccccc} 
& \multicolumn{5}{c}{ Occ. MO } \\
\cline { 2 - 6 } & $\mathbf{2 4}$ & $\mathbf{2 5}$ & $\mathbf{2 6}$ & $\mathbf{2 7}$ & $\mathbf{2 8}$ \\
\hline C1 & 0.26 & 0.84 & 0.29 & 0.81 & 12.68 \\
O2 & 2.83 & 0.72 & 37.87 & 43.11 & 23.91 \\
O3 & 0.72 & 1.63 & 42.72 & 50.10 & 19.29 \\
C4 & 25.29 & 23.74 & 4.53 & 0.50 & 34.90 \\
C5 & 5.18 & 31.75 & 0.36 & 2.21 & 2.28
\end{tabular}




\begin{tabular}{lccccc}
$\mathbf{H 6}$ & 4.24 & 1.92 & 0.05 & 0.10 & 0.03 \\
$\mathbf{H 7}$ & 0.00 & 0.03 & 0.00 & 1.20 & 0.65 \\
$\mathbf{H} 8$ & 3.71 & 2.40 & 0.05 & 0.11 & 0.02 \\
$\mathbf{C 9}$ & 22.61 & 12.71 & 6.84 & 0.73 & 1.63 \\
$\mathbf{H} 10$ & 5.23 & 0.87 & 0.00 & 0.00 & 0.18 \\
$\mathbf{H 1 1}$ & 0.15 & 5.78 & 0.11 & 0.03 & 0.06 \\
$\mathbf{H 1 2}$ & 0.05 & 0.00 & 0.12 & 0.16 & 1.24 \\
$\mathbf{C 1 3}$ & 24.52 & 10.76 & 6.83 & 0.73 & 1.62 \\
$\mathbf{H 1 4}$ & 0.36 & 5.58 & 0.11 & 0.03 & 0.07 \\
$\mathbf{H 1 5}$ & 4.79 & 1.26 & 0.00 & 0.00 & 0.18 \\
$\mathbf{H 1 6}$ & 0.06 & 0.00 & 0.11 & 0.17 & 1.24 \\
\hline \%COO- & 3.81 & 3.19 & 80.88 & 94.02 & 55.89
\end{tabular}

Compound 2:

\begin{tabular}{lccccc} 
& \multicolumn{5}{c}{ Occ. MO } \\
\cline { 2 - 6 } & $\mathbf{1 6}$ & $\mathbf{1 7}$ & $\mathbf{1 8}$ & $\mathbf{1 9}$ & $\mathbf{2 0}$ \\
\hline C1 & 4.35 & 2.10 & 0.33 & 4.21 & 8.11 \\
O2 & 5.40 & 9.21 & 47.11 & 62.98 & 10.95 \\
O3 & 2.50 & 3.44 & 51.48 & 23.00 & 42.40 \\
C4 & 24.44 & 24.35 & 0.35 & 6.13 & 32.34 \\
H5 & 4.59 & 12.41 & 0.04 & 0.47 & 0.17 \\
H6 & 4.53 & 12.41 & 0.04 & 0.47 & 0.17 \\
C7 & 36.90 & 17.81 & 0.14 & 2.47 & 4.62 \\
H8 & 0.42 & 9.16 & 0.25 & 0.12 & 0.39 \\
H9 & 0.42 & 9.11 & 0.25 & 0.12 & 0.40 \\
H10 & 16.44 & 0.01 & 0.00 & 0.05 & 0.44 \\
\hline \%COO- & 12.25 & 14.74 & 98.92 & 90.18 & 61.46
\end{tabular}

Compound 3:

\begin{tabular}{lccccc} 
& \multicolumn{5}{c}{ Occ. MO } \\
\cline { 2 - 6 } & $\mathbf{2 0}$ & $\mathbf{2 1}$ & $\mathbf{2 2}$ & $\mathbf{2 3}$ & $\mathbf{2 4}$ \\
\hline $\mathbf{C 1}$ & 2.23 & 1.22 & 0.42 & 2.02 & 9.92 \\
O2 & 4.35 & 4.41 & 47.18 & 45.33 & 25.28 \\
O3 & 3.26 & 0.69 & 47.71 & 45.06 & 24.27 \\
C4 & 17.30 & 15.13 & 0.72 & 1.65 & 34.96 \\
H5 & 0.26 & 11.71 & 0.02 & 0.50 & 0.38 \\
H6 & 10.35 & 2.42 & 0.05 & 0.20 & 0.43 \\
C7 & 23.47 & 24.14 & 1.81 & 4.25 & 2.90 \\
H8 & 4.14 & 0.08 & 0.06 & 0.51 & 1.09 \\
H9 & 1.57 & 17.52 & 0.16 & 0.01 & 0.07 \\
C10 & 22.88 & 13.90 & 1.43 & 0.38 & 0.40 \\
H11 & 5.37 & 1.18 & 0.15 & 0.03 & 0.06 \\
H12 & 2.74 & 0.02 & 0.17 & 0.02 & 0.21 \\
H13 & 2.08 & 7.56 & 0.13 & 0.03 & 0.02 \\
\hline \%C00 & 9.84 & 6.32 & 95.31 & 92.40 & 59.47
\end{tabular}


Compound 4:

\begin{tabular}{lccccc} 
& \multicolumn{5}{c}{ Occ. MO } \\
\cline { 2 - 6 } & $\mathbf{1 2}$ & $\mathbf{1 3}$ & $\mathbf{1 4}$ & $\mathbf{1 5}$ & $\mathbf{1 6}$ \\
\hline $\mathbf{C 1}$ & 7.69 & 11.32 & 0.35 & 0.98 & 6.63 \\
$\mathbf{O 2}$ & 12.92 & 19.55 & 48.32 & 44.26 & 35.03 \\
$\mathbf{O 3}$ & 14.77 & 16.23 & 51.07 & 49.98 & 30.02 \\
$\mathbf{C 4}$ & 35.03 & 27.46 & 0.19 & 1.72 & 26.71 \\
$\mathbf{H 5}$ & 4.92 & 12.75 & 0.04 & 0.52 & 0.43 \\
$\mathbf{H 6}$ & 5.01 & 12.69 & 0.04 & 0.53 & 0.43 \\
$\mathbf{H 7}$ & 19.65 & 0.01 & 0.00 & 2.02 & 0.76 \\
\hline \% OOO $^{-}$ & 35.38 & 47.10 & 99.74 & 95.22 & 71.68
\end{tabular}

\section{Compound 5:}

\begin{tabular}{lccccc} 
& \multicolumn{5}{c}{ Occ. MO } \\
\cline { 2 - 6 } & $\mathbf{3 2}$ & $\mathbf{3 3}$ & $\mathbf{3 4}$ & $\mathbf{3 5}$ & $\mathbf{3 6}$ \\
\hline C1 & 0.33 & 1.24 & 22.60 & 0.03 & 0.58 \\
O2 & 47.48 & 26.01 & 6.78 & 3.74 & 3.47 \\
O3 & 47.30 & 13.25 & 17.17 & 4.70 & 2.80 \\
C4 & 0.01 & 8.86 & 45.49 & 0.18 & 29.08 \\
C5 & 1.53 & 19.43 & 2.84 & 23.68 & 4.37 \\
C6 & 0.92 & 23.18 & 1.73 & 22.10 & 9.54 \\
C7 & 0.01 & 2.03 & 1.05 & 0.20 & 27.39 \\
C8 & 0.94 & 2.49 & 0.78 & 25.02 & 6.65 \\
C9 & 1.47 & 3.33 & 0.83 & 20.20 & 7.53 \\
H10 & 0.00 & 0.08 & 0.03 & 0.01 & 0.00 \\
H11 & 0.00 & 0.02 & 0.12 & 0.01 & 0.00 \\
H12 & 0.00 & 0.00 & 0.13 & 0.01 & 0.00 \\
H13 & 0.00 & 0.03 & 0.08 & 0.01 & 0.00 \\
C14 & 0.00 & 0.02 & 0.34 & 0.02 & 3.01 \\
H15 & 0.00 & 0.00 & 0.00 & 0.04 & 2.80 \\
H16 & 0.00 & 0.00 & 0.00 & 0.04 & 2.76 \\
H17 & 0.00 & 0.01 & 0.00 & 0.00 & 0.00 \\
\hline \%C0O- & 95.11 & 40.50 & 46.56 & 8.47 & 6.85
\end{tabular}

\section{Compound 6:}

\begin{tabular}{lccccc} 
& \multicolumn{5}{c}{ Occ. MO } \\
\cline { 2 - 6 } & $\mathbf{1 9}$ & $\mathbf{2 0}$ & $\mathbf{2 1}$ & $\mathbf{2 2}$ & $\mathbf{2 3}$ \\
\hline C1 & 1.01 & 2.51 & 2.58 & 2.17 & 7.39 \\
O2 & 0.48 & 32.81 & 33.34 & 26.06 & 23.90 \\
O3 & 3.02 & 29.21 & 22.16 & 55.94 & 12.00 \\
C4 & 20.66 & 5.48 & 17.51 & 3.62 & 24.76 \\
H5 & 0.11 & 1.15 & 0.54 & 0.14 & 1.99 \\
H6 & 6.76 & 0.67 & 0.07 & 1.48 & 0.03 \\
C7 & 24.09 & 15.74 & 15.46 & 6.69 & 10.46 \\
C8 & 11.46 & 12.13 & 8.04 & 3.81 & 19.29 \\
H9 & 16.04 & 0.05 & 0.05 & 0.03 & 0.00 \\
H10 & 6.72 & 0.21 & 0.20 & 0.03 & 0.13 \\
H11 & 9.64 & 0.03 & 0.05 & 0.05 & 0.04 \\
\hline
\end{tabular}


$\begin{array}{llllll}\% \mathrm{COO}^{-} & 4.51 & 64.53 & 58.08 & 84.16 & 43.29\end{array}$

Compound 7:

Occ. MO

\begin{tabular}{lccccc} 
& $\mathbf{3 2}$ & $\mathbf{3 3}$ & $\mathbf{3 4}$ & $\mathbf{3 5}$ & $\mathbf{3 6}$ \\
\hline $\mathbf{C 1}$ & 0.70 & 1.49 & 0.51 & 2.52 & 9.24 \\
O2 & 45.42 & 3.93 & 13.07 & 48.50 & 8.72 \\
O3 & 42.77 & 19.50 & 6.50 & 16.17 & 17.12 \\
$\mathbf{C 4}$ & 1.55 & 20.34 & 10.50 & 4.39 & 15.20 \\
$\mathbf{H 5}$ & 0.12 & 0.05 & 0.46 & 1.77 & 0.05 \\
$\mathbf{H 6}$ & 0.05 & 0.14 & 0.17 & 0.32 & 1.84 \\
$\mathbf{C 7}$ & 1.57 & 14.75 & 22.35 & 2.18 & 13.62 \\
$\mathbf{C 8}$ & 2.43 & 14.53 & 11.71 & 8.18 & 9.82 \\
$\mathbf{C 9}$ & 1.21 & 10.39 & 11.91 & 4.93 & 7.04 \\
$\mathbf{C 1 0}$ & 2.03 & 6.41 & 0.67 & 0.79 & 9.88 \\
$\mathbf{C 1 1}$ & 1.57 & 5.93 & 10.22 & 6.90 & 2.08 \\
$\mathbf{C 1 2}$ & 0.55 & 2.41 & 11.75 & 2.95 & 5.29 \\
$\mathbf{H 1 3}$ & 0.01 & 0.05 & 0.10 & 0.25 & 0.08 \\
$\mathbf{H 1 4}$ & 0.00 & 0.01 & 0.03 & 0.05 & 0.00 \\
$\mathbf{H 1 5}$ & 0.00 & 0.02 & 0.01 & 0.03 & 0.01 \\
$\mathbf{H 1 6}$ & 0.00 & 0.04 & 0.01 & 0.02 & 0.02 \\
$\mathbf{H 1 7}$ & 0.03 & 0.02 & 0.02 & 0.02 & 0.00 \\
\hline \%COO- & 88.89 & 24.93 & 20.08 & 67.20 & 35.08
\end{tabular}

Compound 8:

Occ. MO

\begin{tabular}{lccccc} 
& $\mathbf{3 2}$ & $\mathbf{3 3}$ & $\mathbf{3 4}$ & $\mathbf{3 5}$ & $\mathbf{3 6}$ \\
\hline $\mathbf{C 1}$ & 0.34 & 17.36 & 5.75 & 0.49 & 0.15 \\
O2 & 49.40 & 3.50 & 20.80 & 0.70 & 3.86 \\
O3 & 47.41 & 28.73 & 3.55 & 6.79 & 0.25 \\
C4 & 0.05 & 39.11 & 23.91 & 25.85 & 5.13 \\
C5 & 1.14 & 3.52 & 31.54 & 21.91 & 9.73 \\
C6 & 0.33 & 3.66 & 13.18 & 0.16 & 29.05 \\
C7 & 0.01 & 0.99 & 0.33 & 23.29 & 7.73 \\
C8 & 0.46 & 1.40 & 0.38 & 20.49 & 7.85 \\
C9 & 0.68 & 1.10 & 0.30 & 0.20 & 27.04 \\
H10 & 0.00 & 0.01 & 0.07 & 0.01 & 0.00 \\
H11 & 0.00 & 0.02 & 0.01 & 0.01 & 0.00 \\
H12 & 0.00 & 0.14 & 0.04 & 0.01 & 0.00 \\
H13 & 0.00 & 0.11 & 0.01 & 0.00 & 0.01 \\
C14 & 0.07 & 0.33 & 0.12 & 0.03 & 3.23 \\
H15 & 0.06 & 0.00 & 0.00 & 0.03 & 2.98 \\
H16 & 0.06 & 0.00 & 0.00 & 0.03 & 2.98 \\
H17 & 0.00 & 0.02 & 0.03 & 0.00 & 0.00 \\
\hline \%COO & 97.15 & 49.58 & 30.09 & 7.99 & 4.26
\end{tabular}


Compound 9:

\begin{tabular}{lccccc} 
& \multicolumn{5}{c}{ Occ. MO } \\
\cline { 2 - 6 } & $\mathbf{2 7}$ & $\mathbf{2 8}$ & $\mathbf{2 9}$ & $\mathbf{3 0}$ & $\mathbf{3 1}$ \\
\hline $\mathbf{C 1}$ & 3.06 & 3.36 & 0.25 & 1.33 & 3.40 \\
O2 & 35.36 & 63.39 & 0.04 & 1.14 & 1.66 \\
O3 & 43.60 & 15.00 & 0.04 & 0.15 & 0.67 \\
H4 & 0.03 & 0.16 & 0.00 & 0.00 & 0.02 \\
C5 & 4.48 & 9.80 & 4.46 & 2.01 & 8.78 \\
H6 & 0.01 & 1.21 & 0.01 & 0.06 & 0.08 \\
H7 & 0.54 & 0.07 & 0.18 & 0.01 & 0.03 \\
C8 & 7.56 & 1.19 & 7.99 & 7.35 & 36.66 \\
H9 & 0.32 & 0.13 & 0.02 & 0.74 & 0.11 \\
H10 & 1.33 & 0.01 & 0.01 & 0.07 & 0.25 \\
C11 & 2.55 & 0.65 & 0.89 & 5.62 & 11.35 \\
O12 & 0.75 & 0.42 & 42.69 & 56.75 & 9.83 \\
O13 & 0.39 & 4.62 & 43.41 & 24.76 & 27.16 \\
\hline \%C00 & 3.69 & 5.69 & 86.99 & 87.13 & 48.34
\end{tabular}

\section{Compound 10:}

\begin{tabular}{lccccc} 
& \multicolumn{5}{c}{ Occ. MO } \\
\cline { 2 - 6 } & $\mathbf{2 8}$ & $\mathbf{2 9}$ & $\mathbf{3 0}$ & $\mathbf{3 1}$ & $\mathbf{3 2}$ \\
\hline C1 & 1.00 & 2.57 & 23.40 & 4.90 & 6.71 \\
C2 & 1.61 & 5.72 & 21.45 & 12.12 & 8.30 \\
C3 & 0.01 & 44.69 & 0.66 & 8.26 & 32.39 \\
C4 & 1.60 & 1.06 & 21.97 & 18.07 & 7.67 \\
C5 & 1.01 & 0.32 & 22.71 & 7.78 & 7.32 \\
C6 & 0.00 & 0.96 & 0.08 & 0.18 & 29.58 \\
H7 & 0.00 & 0.15 & 0.01 & 0.01 & 0.00 \\
H8 & 0.00 & 0.09 & 0.01 & 0.03 & 0.00 \\
H9 & 0.00 & 0.03 & 0.01 & 0.11 & 0.00 \\
H10 & 0.00 & 0.13 & 0.01 & 0.04 & 0.00 \\
H11 & 0.00 & 0.02 & 0.00 & 0.00 & 0.01 \\
C12 & 0.33 & 16.44 & 0.25 & 2.98 & 0.59 \\
O13 & 47.20 & 6.20 & 4.38 & 32.54 & 3.85 \\
O14 & 47.23 & 21.61 & 5.06 & 12.99 & 3.58 \\
\hline \%C00 & 94.76 & 44.25 & 9.69 & 48.51 & 8.02
\end{tabular}

\section{Compound 11:}

\begin{tabular}{lccccc} 
& \multicolumn{5}{c}{ Occ. MO } \\
\cline { 2 - 6 } & $\mathbf{3 2}$ & $\mathbf{3 3}$ & $\mathbf{3 4}$ & $\mathbf{3 5}$ & $\mathbf{3 6}$ \\
\hline C1 & 0.29 & 0.08 & 20.18 & 0.47 & 0.36 \\
O2 & 41.35 & 12.57 & 13.00 & 26.58 & 3.23 \\
O3 & 41.35 & 12.58 & 13.62 & 25.67 & 3.22 \\
C4 & 0.02 & 0.04 & 49.08 & 3.53 & 27.99 \\
C5 & 4.54 & 16.90 & 1.78 & 16.48 & 6.59 \\
C6 & 3.94 & 20.42 & 0.43 & 16.38 & 7.86 \\
C7 & 0.02 & 0.07 & 0.13 & 2.58 & 24.67
\end{tabular}




\begin{tabular}{lccccc} 
C8 & 3.93 & 20.42 & 0.41 & 3.16 & 7.86 \\
C9 & 4.55 & 16.89 & 1.02 & 4.94 & 6.60 \\
H10 & 0.00 & 0.01 & 0.06 & 0.07 & 0.00 \\
H11 & 0.00 & 0.01 & 0.11 & 0.00 & 0.00 \\
H12 & 0.00 & 0.01 & 0.11 & 0.00 & 0.00 \\
H13 & 0.00 & 0.01 & 0.06 & 0.08 & 0.00 \\
F14 & 0.00 & 0.00 & 0.02 & 0.04 & 11.60 \\
\hline \%(00 & 82.99 & 25.23 & 46.80 & 52.73 & 6.82
\end{tabular}

\section{Compound 12:}

\begin{tabular}{lccccc} 
& \multicolumn{5}{c}{ Occ. MO } \\
\cline { 2 - 6 } & $\mathbf{2 4}$ & $\mathbf{2 5}$ & $\mathbf{2 6}$ & $\mathbf{2 7}$ & $\mathbf{2 8}$ \\
\hline C1 & 0.74 & 0.03 & 0.34 & 1.92 & 11.90 \\
O2 & 1.27 & 0.98 & 47.55 & 45.06 & 18.95 \\
O3 & 1.34 & 0.89 & 48.17 & 45.45 & 16.86 \\
C4 & 7.75 & 0.22 & 0.32 & 1.29 & 42.03 \\
H5 & 0.16 & 0.59 & 0.05 & 0.25 & 0.22 \\
H6 & 0.17 & 0.59 & 0.05 & 0.25 & 0.23 \\
C7 & 3.15 & 3.79 & 0.74 & 3.35 & 5.70 \\
H8 & 0.91 & 3.48 & 0.35 & 0.03 & 0.01 \\
H9 & 0.97 & 3.46 & 0.35 & 0.03 & 0.01 \\
Cl10 & 83.54 & 85.99 & 2.09 & 2.38 & 4.10 \\
\hline \%COO- & 3.35 & 1.90 & 96.06 & 92.43 & 47.70
\end{tabular}

\section{Compound 13:}

\begin{tabular}{lccccc} 
& \multicolumn{5}{c}{ Occ. MO } \\
\cline { 2 - 6 } & $\mathbf{3 6}$ & $\mathbf{3 7}$ & $\mathbf{3 8}$ & $\mathbf{3 9}$ & $\mathbf{4 0}$ \\
\hline C1 & 3.20 & 21.20 & 0.40 & 12.83 & 6.23 \\
C2 & 3.86 & 17.94 & 1.31 & 19.89 & 4.89 \\
C3 & 0.02 & 0.04 & 48.65 & 0.68 & 23.20 \\
C4 & 3.86 & 17.93 & 1.34 & 19.88 & 4.90 \\
C5 & 3.20 & 21.21 & 0.40 & 12.85 & 6.22 \\
C6 & 0.01 & 0.07 & 0.09 & 0.03 & 22.38 \\
H7 & 0.00 & 0.01 & 0.12 & 0.00 & 0.00 \\
H8 & 0.00 & 0.01 & 0.06 & 0.05 & 0.00 \\
H9 & 0.00 & 0.01 & 0.06 & 0.05 & 0.00 \\
H10 & 0.00 & 0.01 & 0.12 & 0.00 & 0.00 \\
C11 & 0.30 & 0.07 & 20.17 & 0.41 & 0.45 \\
O12 & 42.77 & 10.76 & 13.72 & 16.52 & 2.53 \\
O13 & 42.77 & 10.75 & 13.52 & 16.70 & 2.53 \\
C114 & 0.00 & 0.00 & 0.03 & 0.10 & 26.67 \\
\hline \%C00 & 85.84 & 21.58 & 47.41 & 33.63 & 5.50
\end{tabular}

Compound 14:

\begin{tabular}{cccccc} 
& \multicolumn{5}{c}{ Occ. MO } \\
\cline { 2 - 6 } & $\mathbf{4 5}$ & $\mathbf{4 6}$ & $\mathbf{4 7}$ & $\mathbf{4 8}$ & $\mathbf{4 9}$ \\
\hline C1 & 0.30 & 0.07 & 19.27 & 0.52 & 0.30
\end{tabular}




\begin{tabular}{lccccc} 
O2 & 42.16 & 11.54 & 14.87 & 23.22 & 2.20 \\
O3 & 42.16 & 11.54 & 13.51 & 24.89 & 2.21 \\
C4 & 0.02 & 0.04 & 47.98 & 3.73 & 19.86 \\
C5 & 4.17 & 17.51 & 1.81 & 18.13 & 4.24 \\
C6 & 3.50 & 20.85 & 0.43 & 15.36 & 6.17 \\
C7 & 0.02 & 0.07 & 0.08 & 4.22 & 18.45 \\
C8 & 3.50 & 20.84 & 0.49 & 3.87 & 6.18 \\
C9 & 4.16 & 17.51 & 1.09 & 5.70 & 4.23 \\
H10 & 0.00 & 0.01 & 0.07 & 0.07 & 0.00 \\
H11 & 0.00 & 0.01 & 0.13 & 0.00 & 0.00 \\
H12 & 0.00 & 0.01 & 0.13 & 0.00 & 0.00 \\
H13 & 0.00 & 0.01 & 0.07 & 0.08 & 0.00 \\
Br14 & 0.00 & 0.01 & 0.07 & 0.24 & 36.16 \\
\hline \%COO- & 84.62 & 23.15 & 47.65 & 48.62 & 4.71
\end{tabular}

\section{Compound 15:}

\begin{tabular}{lccccc} 
& \multicolumn{5}{c}{ Occ. MO } \\
\cline { 2 - 6 } & $\mathbf{3 2}$ & $\mathbf{3 3}$ & $\mathbf{3 4}$ & $\mathbf{3 5}$ & $\mathbf{3 6}$ \\
\hline C1 & 0.34 & 0.43 & 17.32 & 0.61 & 0.09 \\
O2 & 48.50 & 0.07 & 10.93 & 27.93 & 5.88 \\
O3 & 43.61 & 11.71 & 12.75 & 25.04 & 0.30 \\
C4 & 0.26 & 21.29 & 48.33 & 1.92 & 7.83 \\
C5 & 2.96 & 23.91 & 3.37 & 21.73 & 6.39 \\
C6 & 0.76 & 0.19 & 1.43 & 20.12 & 25.32 \\
C7 & 0.19 & 21.28 & 3.71 & 0.97 & 9.99 \\
C8 & 1.67 & 20.98 & 0.97 & 0.55 & 5.45 \\
C9 & 1.26 & 0.06 & 0.67 & 0.97 & 26.68 \\
H10 & 0.00 & 0.01 & 0.07 & 0.07 & 0.00 \\
H11 & 0.00 & 0.01 & 0.01 & 0.00 & 0.00 \\
H12 & 0.00 & 0.01 & 0.19 & 0.00 & 0.00 \\
H13 & 0.00 & 0.00 & 0.04 & 0.07 & 0.01 \\
F14 & 0.46 & 0.04 & 0.21 & 0.04 & 12.07 \\
\hline \%C00 & 92.44 & 12.22 & 41.00 & 53.58 & 6.27
\end{tabular}

Compound 16:

\begin{tabular}{lccccc} 
& \multicolumn{5}{c}{ Occ. MO } \\
\cline { 2 - 6 } & $\mathbf{3 6}$ & $\mathbf{3 7}$ & $\mathbf{3 8}$ & $\mathbf{3 9}$ & $\mathbf{4 0}$ \\
\hline C1 & 1.15 & 21.31 & 6.35 & 1.23 & 3.98 \\
C2 & 0.79 & 0.08 & 10.36 & 6.30 & 21.15 \\
C3 & 0.32 & 21.45 & 16.16 & 25.08 & 6.39 \\
C4 & 2.43 & 24.17 & 20.55 & 29.98 & 5.16 \\
C5 & 0.44 & 0.20 & 7.79 & 8.87 & 22.72 \\
C6 & 0.19 & 21.30 & 6.43 & 0.70 & 8.00 \\
H7 & 0.00 & 0.01 & 0.07 & 0.06 & 0.00 \\
H8 & 0.00 & 0.00 & 0.07 & 0.01 & 0.01 \\
H9 & 0.00 & 0.01 & 0.01 & 0.06 & 0.00 \\
C10 & 0.34 & 0.43 & 7.51 & 9.94 & 0.16
\end{tabular}




\begin{tabular}{lccccc}
$\mathbf{0 1 1}$ & 49.35 & 0.03 & 3.27 & 15.58 & 4.25 \\
O12 & 43.77 & 10.88 & 21.20 & 1.84 & 0.17 \\
H13 & 0.00 & 0.01 & 0.00 & 0.00 & 0.00 \\
Cl14 & 1.20 & 0.13 & 0.23 & 0.35 & 28.00 \\
\hline \%COO & 93.46 & 11.33 & 31.98 & 27.36 & 4.57
\end{tabular}

Compound 17:

\begin{tabular}{lccccc} 
& \multicolumn{5}{c}{ Occ. MO } \\
\cline { 2 - 6 } & $\mathbf{1 6}$ & $\mathbf{1 7}$ & $\mathbf{1 8}$ & $\mathbf{1 9}$ & $\mathbf{2 0}$ \\
\hline C1 & 10.40 & 0.20 & 0.19 & 1.23 & 6.16 \\
O2 & 4.58 & 28.16 & 19.23 & 42.58 & 34.32 \\
O3 & 2.70 & 15.74 & 34.85 & 52.36 & 27.66 \\
C4 & 16.20 & 4.10 & 4.54 & 0.76 & 27.47 \\
H5 & 2.68 & 4.97 & 3.53 & 0.18 & 0.56 \\
H6 & 2.67 & 4.97 & 3.58 & 0.17 & 0.56 \\
O7 & 57.42 & 41.84 & 34.06 & 2.42 & 2.66 \\
H8 & 3.37 & 0.03 & 0.02 & 0.31 & 0.62 \\
\hline \%COO- & 17.68 & 44.10 & 54.27 & 96.16 & 68.14
\end{tabular}

Compound 18:

\begin{tabular}{lccccc} 
& \multicolumn{5}{c}{ Occ. MO } \\
\cline { 2 - 6 } & $\mathbf{4 5}$ & $\mathbf{4 6}$ & $\mathbf{4 7}$ & $\mathbf{4 8}$ & $\mathbf{4 9}$ \\
\hline C1 & 0.35 & 0.44 & 3.28 & 12.57 & 0.10 \\
O2 & 50.17 & 0.03 & 13.17 & 19.67 & 3.34 \\
O3 & 43.06 & 11.14 & 37.99 & 4.64 & 0.13 \\
C4 & 0.50 & 21.78 & 7.90 & 45.93 & 5.19 \\
C5 & 2.32 & 23.77 & 16.88 & 6.98 & 5.31 \\
C6 & 0.23 & 0.15 & 18.15 & 3.70 & 18.51 \\
C7 & 0.28 & 21.71 & 0.08 & 4.50 & 7.43 \\
C8 & 0.93 & 20.77 & 0.98 & 0.54 & 3.55 \\
C9 & 0.45 & 0.06 & 1.31 & 0.42 & 18.35 \\
H10 & 0.00 & 0.01 & 0.02 & 0.08 & 0.00 \\
H11 & 0.00 & 0.01 & 0.00 & 0.01 & 0.00 \\
H12 & 0.00 & 0.01 & 0.05 & 0.13 & 0.00 \\
H13 & 0.00 & 0.00 & 0.12 & 0.02 & 0.01 \\
Br14 & 1.72 & 0.12 & 0.07 & 0.81 & 38.07 \\
\hline \%C00 & 93.57 & 11.61 & 54.44 & 36.88 & 3.57
\end{tabular}

Compound 19:

Occ. MO

\begin{tabular}{lccccc} 
& $\mathbf{8}$ & $\mathbf{9}$ & $\mathbf{1 0}$ & $\mathbf{1 1}$ & $\mathbf{1 2}$ \\
\hline $\mathbf{C 1}$ & 21.33 & 34.59 & 0.34 & 1.49 & 6.69 \\
O2 & 39.53 & 32.57 & 49.89 & 48.38 & 37.50 \\
O3 & 39.13 & 32.83 & 49.77 & 50.12 & 35.76 \\
H4 & 0.00 & 0.01 & 0.00 & 0.01 & 20.05 \\
\hline \% OOO $^{-}$ & 100.00 & 99.99 & 100.00 & 99.99 & 79.95
\end{tabular}


Compound 20:

Occ. MO

\begin{tabular}{lccccc} 
& $\mathbf{3 4}$ & $\mathbf{3 5}$ & $\mathbf{3 6}$ & $\mathbf{3 7}$ & $\mathbf{3 8}$ \\
\hline $\mathbf{C 1}$ & 0.29 & 0.49 & 0.29 & 14.12 & 0.77 \\
O2 & 41.66 & 0.69 & 14.25 & 10.85 & 19.19 \\
O3 & 37.78 & 16.10 & 3.46 & 9.72 & 20.73 \\
C4 & 0.19 & 21.40 & 8.39 & 51.42 & 2.35 \\
C5 & 5.93 & 20.93 & 2.94 & 8.73 & 32.13 \\
C6 & 3.24 & 0.11 & 24.23 & 1.71 & 22.13 \\
C7 & 0.26 & 21.67 & 6.23 & 1.20 & 0.27 \\
C8 & 4.53 & 18.39 & 4.91 & 0.63 & 0.48 \\
C9 & 3.67 & 0.17 & 20.82 & 0.54 & 0.80 \\
H10 & 0.00 & 0.01 & 0.00 & 0.05 & 0.04 \\
H11 & 0.00 & 0.01 & 0.00 & 0.01 & 0.00 \\
H12 & 0.00 & 0.01 & 0.00 & 0.13 & 0.00 \\
H13 & 0.00 & 0.00 & 0.01 & 0.04 & 0.06 \\
C14 & 0.28 & 0.02 & 1.34 & 0.73 & 1.04 \\
N15 & 2.17 & 0.00 & 13.15 & 0.11 & 0.02 \\
\hline \%C00 & 79.74 & 17.28 & 17.99 & 34.69 & 40.68
\end{tabular}

Compound 21:

Occ. MO

\begin{tabular}{lccccc} 
& $\mathbf{3 4}$ & $\mathbf{3 5}$ & $\mathbf{3 6}$ & $\mathbf{3 7}$ & $\mathbf{3 8}$ \\
\hline C1 & 0.23 & 0.16 & 0.60 & 22.32 & 0.48 \\
O2 & 30.70 & 24.53 & 3.10 & 11.97 & 20.06 \\
O3 & 30.71 & 24.55 & 3.07 & 12.34 & 19.61 \\
C4 & 0.04 & 0.02 & 25.85 & 46.83 & 3.30 \\
C5 & 9.66 & 11.07 & 7.20 & 1.57 & 22.32 \\
C6 & 9.47 & 14.26 & 4.88 & 0.37 & 25.00 \\
C7 & 0.03 & 0.05 & 26.94 & 1.39 & 2.17 \\
C8 & 9.46 & 14.28 & 4.87 & 0.43 & 2.86 \\
C9 & 9.67 & 11.05 & 7.21 & 0.93 & 4.03 \\
H10 & 0.01 & 0.00 & 0.00 & 0.06 & 0.05 \\
H11 & 0.00 & 0.01 & 0.00 & 0.10 & 0.00 \\
H12 & 0.00 & 0.01 & 0.00 & 0.10 & 0.00 \\
H13 & 0.01 & 0.00 & 0.00 & 0.06 & 0.05 \\
C14 & 0.00 & 0.00 & 1.46 & 1.48 & 0.05 \\
N15 & 0.00 & 0.00 & 14.81 & 0.07 & 0.03 \\
\hline \%C00 & 61.64 & 49.25 & 6.77 & 46.63 & 40.15
\end{tabular}

Compound 22:

Occ. MO

\begin{tabular}{lccccc} 
& $\mathbf{2 0}$ & $\mathbf{2 1}$ & $\mathbf{2 2}$ & $\mathbf{2 3}$ & $\mathbf{2 4}$ \\
\hline C1 & 2.57 & 0.31 & 3.57 & 0.08 & 12.09 \\
O2 & 1.47 & 41.99 & 42.82 & 4.74 & 27.46 \\
O3 & 0.64 & 39.27 & 47.69 & 11.61 & 25.50 \\
C4 & 9.27 & 0.33 & 2.33 & 3.62 & 31.00
\end{tabular}




\begin{tabular}{lccccc} 
H5 & 1.50 & 1.09 & 0.14 & 4.67 & 0.43 \\
H6 & 1.51 & 1.10 & 0.22 & 4.67 & 0.41 \\
O7 & 57.39 & 12.43 & 2.35 & 57.19 & 1.79 \\
C8 & 12.36 & 0.98 & 0.86 & 2.64 & 0.96 \\
H9 & 2.99 & 1.25 & 0.01 & 5.38 & 0.01 \\
H10 & 3.01 & 1.25 & 0.00 & 5.40 & 0.01 \\
$\mathbf{H 1 1}$ & 7.28 & 0.00 & 0.02 & 0.00 & 0.33 \\
\hline \% OO- $^{-}$ & 4.69 & 81.57 & 94.08 & 16.43 & 65.06
\end{tabular}

\section{Compound 23:}

\begin{tabular}{lccccc} 
& \multicolumn{5}{c}{ Occ. MO } \\
\cline { 2 - 6 } & $\mathbf{1 8}$ & $\mathbf{1 9}$ & $\mathbf{2 0}$ & $\mathbf{2 1}$ & $\mathbf{2 2}$ \\
\hline C1 & 0.79 & 0.30 & 0.11 & 2.19 & 8.25 \\
O2 & 1.43 & 42.15 & 10.13 & 37.53 & 11.65 \\
O3 & 6.22 & 37.28 & 19.36 & 21.00 & 21.72 \\
C4 & 36.77 & 0.78 & 4.77 & 8.17 & 22.27 \\
H5 & 0.13 & 1.13 & 3.06 & 0.22 & 0.46 \\
H6 & 0.13 & 1.12 & 3.02 & 0.22 & 0.46 \\
C7 & 20.82 & 8.57 & 26.37 & 9.29 & 15.22 \\
C8 & 33.71 & 8.67 & 33.15 & 21.36 & 19.89 \\
H9 & 0.01 & 0.01 & 0.02 & 0.01 & 0.08 \\
\hline \%CO0 & 8.43 & 79.73 & 29.60 & 60.73 & 41.61
\end{tabular}

\section{Compound 24:}

\begin{tabular}{lccccc} 
& \multicolumn{5}{c}{ Occ. MO } \\
\cline { 2 - 6 } & $\mathbf{2 6}$ & $\mathbf{2 7}$ & $\mathbf{2 8}$ & $\mathbf{2 9}$ & $\mathbf{3 0}$ \\
\hline C1 & 14.64 & 32.06 & 1.15 & 25.34 & 26.97 \\
C2 & 16.77 & 39.99 & 0.69 & 3.83 & 13.09 \\
H3 & 0.00 & 0.01 & 0.00 & 1.11 & 0.01 \\
H4 & 0.00 & 0.01 & 0.00 & 0.05 & 0.09 \\
C5 & 1.72 & 0.45 & 0.02 & 2.50 & 3.14 \\
O6 & 49.46 & 12.21 & 0.20 & 0.73 & 2.27 \\
O7 & 12.16 & 1.95 & 0.07 & 0.28 & 0.56 \\
H8 & 0.14 & 0.00 & 0.00 & 0.02 & 0.01 \\
C9 & 1.18 & 1.03 & 0.39 & 12.37 & 5.83 \\
O10 & 2.68 & 11.24 & 43.91 & 6.21 & 43.26 \\
O11 & 1.22 & 1.04 & 53.56 & 47.55 & 4.76 \\
\hline \%C00- & 5.09 & 13.31 & 97.87 & 66.13 & 53.86
\end{tabular}

\section{Compound 25:}

Occ. MO

\begin{tabular}{lccccc} 
& $\mathbf{2 9}$ & $\mathbf{3 0}$ & $\mathbf{3 1}$ & $\mathbf{3 2}$ & $\mathbf{3 3}$ \\
\hline $\mathbf{C 1}$ & 5.57 & 0.27 & 0.10 & 5.34 & 4.34 \\
O2 & 1.57 & 32.69 & 10.40 & 63.95 & 6.49 \\
O3 & 12.38 & 31.11 & 17.48 & 14.45 & 45.12 \\
C4 & 8.07 & 0.44 & 2.19 & 13.43 & 15.36 \\
H5 & 0.34 & 0.76 & 1.46 & 0.61 & 0.15
\end{tabular}




\begin{tabular}{lccccc} 
H6 & 0.33 & 0.75 & 1.44 & 0.61 & 0.15 \\
Br7 & 71.73 & 33.98 & 66.93 & 1.60 & 28.39 \\
\hline$\%$ COO $^{-}$ & 19.53 & 64.07 & 27.98 & 83.75 & 55.95
\end{tabular}

Compound 26:

\begin{tabular}{lccccc} 
& \multicolumn{5}{c}{ Occ. MO } \\
\cline { 2 - 6 } & $\mathbf{2 3}$ & $\mathbf{2 4}$ & $\mathbf{2 5}$ & $\mathbf{2 6}$ & $\mathbf{2 7}$ \\
\hline $\mathbf{C 1}$ & 2.58 & 3.44 & 0.44 & 1.80 & 4.24 \\
O2 & 38.98 & 60.25 & 2.29 & 3.70 & 3.78 \\
O3 & 52.90 & 14.26 & 0.83 & 1.18 & 1.06 \\
H4 & 0.03 & 0.12 & 0.01 & 0.11 & 0.06 \\
C5 & 2.87 & 11.05 & 0.29 & 3.35 & 37.26 \\
H6 & 0.02 & 1.36 & 0.05 & 0.63 & 0.09 \\
H7 & 0.03 & 0.04 & 0.02 & 0.43 & 0.19 \\
C8 & 1.37 & 0.30 & 0.46 & 1.60 & 8.30 \\
O9 & 0.26 & 2.79 & 46.23 & 51.42 & 18.48 \\
O10 & 0.94 & 6.40 & 49.38 & 35.77 & 26.55 \\
\hline \%COO- & 2.57 & 9.48 & 96.07 & 88.79 & 53.33
\end{tabular}

Compound 27:

\begin{tabular}{lccccc} 
& \multicolumn{5}{c}{ Occ. MO } \\
\cline { 2 - 6 } & $\mathbf{2 0}$ & $\mathbf{2 1}$ & $\mathbf{2 2}$ & $\mathbf{2 3}$ & $\mathbf{2 4}$ \\
\hline $\mathbf{C 1}$ & 3.71 & 0.07 & 0.32 & 4.38 & 7.61 \\
$\mathbf{O 2}$ & 2.18 & 6.04 & 38.63 & 57.70 & 13.17 \\
O3 & 6.51 & 1.71 & 49.47 & 27.20 & 37.83 \\
$\mathbf{C 4}$ & 4.81 & 3.39 & 1.06 & 7.18 & 26.96 \\
H5 & 0.56 & 3.03 & 0.34 & 0.47 & 0.26 \\
H6 & 0.55 & 3.03 & 0.34 & 0.48 & 0.26 \\
Cl7 & 81.68 & 82.73 & 9.84 & 2.59 & 13.92 \\
\hline \%C0O- & 12.40 & 7.82 & 88.42 & 89.28 & 58.60
\end{tabular}

Compound 28:

\begin{tabular}{lccccc} 
& \multicolumn{5}{c}{ Occ. MO } \\
\cline { 2 - 6 } & $\mathbf{2 4}$ & $\mathbf{2 5}$ & $\mathbf{2 6}$ & $\mathbf{2 7}$ & $\mathbf{2 8}$ \\
\hline C1 & 1.88 & 0.70 & 0.65 & 1.15 & 5.94 \\
O2 & 3.14 & 1.53 & 45.86 & 53.76 & 18.14 \\
O3 & 4.72 & 1.36 & 42.09 & 37.81 & 30.20 \\
C4 & 8.08 & 5.12 & 2.00 & 2.49 & 30.67 \\
H5 & 0.46 & 3.16 & 0.04 & 1.50 & 0.54 \\
C6 & 2.55 & 5.07 & 4.61 & 2.30 & 2.37 \\
H7 & 1.30 & 0.86 & 0.02 & 0.00 & 0.01 \\
H8 & 0.22 & 0.26 & 0.12 & 0.07 & 0.91 \\
H9 & 0.10 & 0.29 & 0.12 & 0.07 & 0.10 \\
Cl10 & 77.57 & 81.65 & 4.48 & 0.84 & 11.11 \\
\hline \%COO- & 9.74 & 3.60 & 88.60 & 92.73 & 54.28
\end{tabular}


Compound 29:

Occ. MO

\begin{tabular}{lccccc} 
& $\mathbf{1 6}$ & $\mathbf{1 7}$ & $\mathbf{1 8}$ & $\mathbf{1 9}$ & $\mathbf{2 0}$ \\
\hline C1 & 12.30 & 2.09 & 0.35 & 1.44 & 6.07 \\
O2 & 6.66 & 8.65 & 45.51 & 45.83 & 31.78 \\
O3 & 6.96 & 2.79 & 52.92 & 49.59 & 29.57 \\
C4 & 8.52 & 18.75 & 0.40 & 0.76 & 26.71 \\
H5 & 2.18 & 11.69 & 0.07 & 0.14 & 0.61 \\
H6 & 2.18 & 11.70 & 0.07 & 0.14 & 0.61 \\
F7 & 61.20 & 44.31 & 0.67 & 2.10 & 4.65 \\
\hline \%COO- & 25.92 & 13.53 & 98.78 & 96.86 & 67.43
\end{tabular}

Compound 30:

\begin{tabular}{lccccc} 
& \multicolumn{5}{c}{ Occ. MO } \\
\cline { 2 - 6 } & $\mathbf{1 8}$ & $\mathbf{1 9}$ & $\mathbf{2 0}$ & $\mathbf{2 1}$ & $\mathbf{2 2}$ \\
\hline C1 & 5.66 & 0.45 & 0.37 & 2.06 & 6.11 \\
O2 & 2.94 & 3.39 & 46.94 & 43.11 & 20.22 \\
O3 & 5.65 & 0.29 & 51.65 & 41.82 & 20.81 \\
C4 & 6.58 & 10.33 & 0.29 & 4.07 & 42.22 \\
H5 & 0.85 & 5.47 & 0.03 & 0.25 & 0.28 \\
H6 & 0.83 & 5.43 & 0.03 & 0.25 & 0.28 \\
C7 & 31.90 & 26.31 & 0.12 & 7.54 & 7.18 \\
N8 & 45.59 & 48.33 & 0.58 & 0.90 & 2.91 \\
\hline \%COO- & 14.26 & 4.13 & 98.95 & 86.99 & 47.13
\end{tabular}

Compound 31:

\begin{tabular}{lccccc} 
& \multicolumn{5}{c}{ Occ. MO } \\
\cline { 2 - 6 } & $\mathbf{2 3}$ & $\mathbf{2 4}$ & $\mathbf{2 5}$ & $\mathbf{2 6}$ & $\mathbf{2 7}$ \\
\hline C1 & 1.52 & 0.04 & 0.35 & 1.89 & 6.30 \\
O2 & 3.07 & 1.09 & 49.11 & 40.73 & 29.56 \\
O3 & 0.92 & 3.24 & 42.72 & 49.83 & 22.10 \\
C4 & 14.48 & 2.84 & 0.44 & 2.12 & 33.55 \\
H5 & 0.63 & 1.44 & 0.12 & 0.31 & 0.17 \\
H6 & 0.56 & 1.50 & 0.11 & 0.40 & 0.15 \\
N7 & 5.20 & 1.25 & 0.59 & 2.59 & 3.13 \\
O8 & 37.48 & 42.84 & 3.24 & 0.99 & 2.45 \\
O9 & 36.13 & 45.75 & 3.33 & 1.14 & 2.58 \\
\hline \%C00- & 5.51 & 4.38 & 92.18 & 92.45 & 57.97
\end{tabular}

Compound 32:

\begin{tabular}{lccccc} 
& \multicolumn{5}{c}{ Occ. MO } \\
\cline { 2 - 6 } & $\mathbf{2 8}$ & $\mathbf{2 9}$ & $\mathbf{3 0}$ & $\mathbf{3 1}$ & $\mathbf{3 2}$ \\
\hline C1 & 3.41 & 0.08 & 0.37 & 1.38 & 4.76 \\
O2 & 3.54 & 0.19 & 47.64 & 51.38 & 15.61 \\
O3 & 1.29 & 0.20 & 45.25 & 35.45 & 30.48 \\
C4 & 5.41 & 0.23 & 0.73 & 6.80 & 29.19 \\
H5 & 5.15 & 0.00 & 0.00 & 1.39 & 0.63
\end{tabular}




\begin{tabular}{lccccc} 
Cl6 & 40.56 & 49.59 & 3.00 & 1.78 & 9.66 \\
Cl7 & 40.63 & 49.70 & 3.01 & 1.83 & 9.68 \\
\hline$\% \mathrm{COO}^{-}$ & 8.24 & 0.48 & 93.26 & 88.21 & 50.85
\end{tabular}

Compound 33:

\begin{tabular}{lccccc} 
& \multicolumn{5}{c}{ Occ. MO } \\
\cline { 2 - 6 } & $\mathbf{1 9}$ & $\mathbf{2 0}$ & $\mathbf{2 1}$ & $\mathbf{2 2}$ & $\mathbf{2 3}$ \\
\hline C1 & 0.03 & 8.10 & 0.36 & 1.69 & 4.35 \\
O2 & 1.13 & 19.42 & 48.71 & 16.04 & 41.78 \\
O3 & 0.20 & 3.85 & 49.15 & 68.65 & 6.42 \\
C4 & 2.06 & 10.67 & 0.02 & 8.77 & 16.22 \\
O5 & 40.85 & 46.50 & 0.69 & 0.29 & 23.52 \\
O6 & 55.70 & 11.27 & 1.07 & 3.92 & 7.66 \\
H7 & 0.03 & 0.19 & 0.00 & 0.63 & 0.06 \\
\hline \%COO & 1.36 & 31.37 & 98.22 & 86.39 & 52.54
\end{tabular}

Compound 34:

\begin{tabular}{lccccc} 
& \multicolumn{5}{c}{ Occ. MO } \\
\cline { 2 - 6 } & $\mathbf{2 0}$ & $\mathbf{2 1}$ & $\mathbf{2 2}$ & $\mathbf{2 3}$ & $\mathbf{2 4}$ \\
\hline C1 & 11.59 & 9.67 & 0.84 & 1.28 & 5.62 \\
O2 & 11.82 & 7.20 & 47.98 & 39.24 & 35.77 \\
O3 & 10.74 & 5.00 & 50.14 & 55.48 & 22.78 \\
C4 & 0.18 & 16.16 & 0.56 & 1.11 & 25.78 \\
H5 & 0.00 & 12.33 & 0.03 & 0.29 & 2.11 \\
F6 & 26.85 & 28.92 & 0.21 & 2.33 & 3.09 \\
F7 & 38.82 & 20.73 & 0.25 & 0.27 & 4.86 \\
\hline \%COO- & 34.15 & 21.87 & 98.95 & 96.01 & 64.17
\end{tabular}

Compound 35:

\begin{tabular}{lccccc} 
& \multicolumn{5}{c}{ Occ. MO } \\
\cline { 2 - 6 } & $\mathbf{3 6}$ & $\mathbf{3 7}$ & $\mathbf{3 8}$ & $\mathbf{3 9}$ & $\mathbf{4 0}$ \\
\hline $\mathbf{C 1}$ & 0.27 & 0.03 & 0.46 & 1.46 & 6.23 \\
O2 & 1.76 & 0.32 & 39.34 & 37.32 & 15.63 \\
O3 & 0.00 & 0.19 & 45.24 & 38.73 & 14.28 \\
C4 & 0.59 & 0.01 & 3.48 & 1.14 & 43.96 \\
CI5 & 10.82 & 37.53 & 0.09 & 13.71 & 6.78 \\
Cl6 & 43.20 & 30.93 & 5.74 & 3.81 & 6.56 \\
Cl7 & 43.35 & 30.99 & 5.65 & 3.82 & 6.56 \\
\hline \%:00 & 2.03 & 0.54 & 85.04 & 77.51 & 36.14
\end{tabular}

Compound 36:

\begin{tabular}{lccccc} 
& \multicolumn{5}{c}{ Occ. MO } \\
\cline { 2 - 6 } & $\mathbf{2 4}$ & $\mathbf{2 5}$ & $\mathbf{2 6}$ & $\mathbf{2 7}$ & $\mathbf{2 8}$ \\
\hline C1 & 2.37 & 17.38 & 0.38 & 1.47 & 4.30 \\
O2 & 2.43 & 16.70 & 48.19 & 39.46 & 35.05 \\
O3 & 2.30 & 14.84 & 50.08 & 54.73 & 22.17 \\
C4 & 0.00 & 0.03 & 0.22 & 0.83 & 29.76
\end{tabular}




\begin{tabular}{lccccc} 
F5 & 22.57 & 16.40 & 0.08 & 2.45 & 2.36 \\
F6 & 35.16 & 17.33 & 0.52 & 0.53 & 3.18 \\
F7 & 35.16 & 17.32 & 0.53 & 0.53 & 3.18 \\
\hline \% COO $^{-}$ & 7.11 & 48.91 & 98.65 & 95.66 & 61.52
\end{tabular}

S4B) Alcohols and Phenols anions - HF/COSMO/6-31G(d,p)

Compound 37:

Occ. MO

\begin{tabular}{lccccc} 
& $\mathbf{1 7}$ & $\mathbf{1 8}$ & $\mathbf{1 9}$ & $\mathbf{2 0}$ & $\mathbf{2 1}$ \\
\hline $\mathbf{C 1}$ & 18.44 & 14.24 & 14.27 & 5.03 & 5.03 \\
$\mathbf{C 2}$ & 5.09 & 18.52 & 6.50 & 18.86 & 1.50 \\
H3 & 0.25 & 5.78 & 2.14 & 0.27 & 1.01 \\
H4 & 0.25 & 2.55 & 5.37 & 0.92 & 0.36 \\
H5 & 4.31 & 2.80 & 0.13 & 2.06 & 0.15 \\
C6 & 5.09 & 11.82 & 13.18 & 1.39 & 18.95 \\
H7 & 0.26 & 0.01 & 7.91 & 0.38 & 0.90 \\
H8 & 4.31 & 1.33 & 1.60 & 0.14 & 2.07 \\
H9 & 0.25 & 7.85 & 0.05 & 1.00 & 0.28 \\
C10 & 5.09 & 7.15 & 17.87 & 10.28 & 10.07 \\
H11 & 4.30 & 0.28 & 2.66 & 1.12 & 1.10 \\
H12 & 0.25 & 1.48 & 6.46 & 0.00 & 1.28 \\
H13 & 0.25 & 6.10 & 1.83 & 1.27 & 0.01 \\
$\mathbf{0 1 4}$ & 51.86 & 20.11 & 20.05 & 57.26 & 57.29
\end{tabular}

Compound 38:

\begin{tabular}{lccccc} 
& \multicolumn{5}{c}{ Occ. MO } \\
\cline { 2 - 6 } & $\mathbf{1 3}$ & $\mathbf{1 4}$ & $\mathbf{1 5}$ & $\mathbf{1 6}$ & $\mathbf{1 7}$ \\
\hline C1 & 8.69 & 11.59 & 20.17 & 15.26 & 6.66 \\
H2 & 0.23 & 10.34 & 0.01 & 0.04 & 1.88 \\
H3 & 4.81 & 2.29 & 2.52 & 1.70 & 0.51 \\
H4 & 1.57 & 0.76 & 12.95 & 1.18 & 0.19 \\
C5 & 18.37 & 14.38 & 12.66 & 5.02 & 5.69 \\
H6 & 0.14 & 9.47 & 0.00 & 0.01 & 18.09 \\
C7 & 8.71 & 11.60 & 20.17 & 14.79 & 7.18 \\
H8 & 1.59 & 0.77 & 12.92 & 1.20 & 0.16 \\
H9 & 0.23 & 10.35 & 0.01 & 0.03 & 1.90 \\
H10 & 4.83 & 2.28 & 2.52 & 1.65 & 0.56 \\
O11 & 50.81 & 26.17 & 16.09 & 59.13 & 57.16
\end{tabular}

Compound 39:

\begin{tabular}{lccccc} 
& \multicolumn{5}{c}{ Occ. MO } \\
\cline { 2 - 6 } & $\mathbf{1 3}$ & $\mathbf{1 4}$ & $\mathbf{1 5}$ & $\mathbf{1 6}$ & $\mathbf{1 7}$ \\
\hline $\mathbf{C 1}$ & 19.55 & 10.09 & 4.82 & 4.60 & 4.72 \\
$\mathbf{H} 2$ & 0.52 & 1.54 & 3.09 & 3.28 & 15.16 \\
H3 & 0.72 & 2.03 & 2.50 & 18.15 & 0.51 \\
O4 & 49.14 & 12.50 & 7.95 & 60.38 & 59.79
\end{tabular}




$\begin{array}{lccccc}\text { C5 } & 10.30 & 26.02 & 26.44 & 9.63 & 15.35 \\ \text { H6 } & 0.02 & 8.64 & 14.02 & 1.95 & 0.00 \\ \text { H7 } & 0.02 & 5.66 & 16.20 & 0.13 & 1.56 \\ \text { C8 } & 12.46 & 22.50 & 10.15 & 1.45 & 2.21 \\ \text { H9 } & 7.07 & 2.04 & 0.02 & 0.37 & 0.60 \\ \text { H10 } & 0.06 & 3.71 & 8.19 & 0.02 & 0.07 \\ \text { H11 } & 0.15 & 5.26 & 6.63 & 0.05 & 0.03\end{array}$

Compound 40:

\begin{tabular}{lccccc} 
& \multicolumn{5}{c}{ Occ. MO } \\
\cline { 2 - 6 } & $\mathbf{9}$ & $\mathbf{1 0}$ & $\mathbf{1 1}$ & $\mathbf{1 2}$ & $\mathbf{1 3}$ \\
\hline $\mathbf{C 1}$ & 24.61 & 11.44 & 14.91 & 4.95 & 5.61 \\
$\mathbf{H 2}$ & 0.15 & 4.07 & 3.15 & 3.95 & 15.32 \\
$\mathbf{H 3}$ & 0.15 & 3.95 & 3.19 & 3.91 & 15.17 \\
$\mathbf{O 4}$ & 59.35 & 20.84 & 21.36 & 59.69 & 62.16 \\
$\mathbf{C 5}$ & 12.83 & 28.08 & 29.92 & 23.87 & 0.78 \\
H6 & 0.16 & 0.01 & 22.95 & 1.35 & 0.00 \\
H7 & 1.39 & 15.87 & 2.20 & 1.15 & 0.48 \\
H8 & 1.36 & 15.74 & 2.31 & 1.14 & 0.48
\end{tabular}

Compound 41:

\begin{tabular}{lccccc} 
& \multicolumn{5}{c}{ Occ. MO } \\
\cline { 2 - 6 } & $\mathbf{5}$ & $\mathbf{6}$ & $\mathbf{7}$ & $\mathbf{8}$ & $\mathbf{9}$ \\
\hline C1 & 37.86 & 37.93 & 22.61 & 4.84 & 4.90 \\
H2 & 14.09 & 5.53 & 1.58 & 14.68 & 4.56 \\
H3 & 15.39 & 4.34 & 1.57 & 14.22 & 5.11 \\
H4 & 0.03 & 19.50 & 1.58 & 0.01 & 19.13 \\
O5 & 32.63 & 32.70 & 72.66 & 66.25 & 66.30
\end{tabular}

Compound 42:

\begin{tabular}{lccccc} 
& \multicolumn{5}{c}{ Occ. MO } \\
\cline { 2 - 6 } & $\mathbf{2 1}$ & $\mathbf{2 2}$ & $\mathbf{2 3}$ & $\mathbf{2 4}$ & $\mathbf{2 5}$ \\
\hline C1 & 0.04 & 11.50 & 6.37 & 21.97 & 0.04 \\
C2 & 36.66 & 24.33 & 21.09 & 3.08 & 5.88 \\
H3 & 10.35 & 3.89 & 0.25 & 3.24 & 12.47 \\
H4 & 10.26 & 3.87 & 0.27 & 3.27 & 12.32 \\
O5 & 28.91 & 27.21 & 57.23 & 62.33 & 68.22 \\
F6 & 6.56 & 4.05 & 5.89 & 2.46 & 0.53 \\
F7 & 0.75 & 21.08 & 2.96 & 1.19 & 0.00 \\
F8 & 6.46 & 4.06 & 5.94 & 2.46 & 0.54
\end{tabular}

Compound 43:

\begin{tabular}{lccccc} 
& \multicolumn{5}{c}{ Occ. MO } \\
\cline { 2 - 6 } & $\mathbf{2 5}$ & $\mathbf{2 6}$ & $\mathbf{2 7}$ & $\mathbf{2 8}$ & $\mathbf{2 9}$ \\
\hline C1 & 13.97 & 3.02 & 2.94 & 23.81 & 2.05 \\
C2 & 4.79 & 0.47 & 12.36 & 25.83 & 17.08 \\
C3 & 1.31 & 0.11 & 6.58 & 0.07 & 7.95
\end{tabular}




$\begin{array}{lccccc}\mathbf{C} 4 & 4.95 & 2.63 & 12.98 & 26.14 & 16.16 \\ \mathbf{C 5} & 14.24 & 8.03 & 3.34 & 23.96 & 2.04 \\ \mathbf{C 6} & 18.45 & 4.97 & 1.24 & 0.08 & 27.88 \\ \mathbf{H 7} & 0.00 & 0.79 & 1.17 & 0.01 & 0.00 \\ \mathbf{H} 8 & 0.00 & 0.28 & 1.61 & 0.01 & 0.00 \\ \mathbf{H} 9 & 0.00 & 0.87 & 1.59 & 0.01 & 0.00 \\ \mathbf{H} 10 & 0.00 & 0.12 & 1.13 & 0.01 & 0.00 \\ \mathbf{~ O 1 1 ~} & 35.89 & 1.86 & 54.88 & 0.00 & 23.31 \\ \mathbf{N} 12 & 3.53 & 75.05 & 0.17 & 0.03 & 1.30 \\ \mathbf{H} 13 & 1.43 & 0.90 & 0.01 & 0.02 & 1.11 \\ \mathbf{H} 14 & 1.43 & 0.90 & 0.01 & 0.02 & 1.11\end{array}$

Compound 44:

\begin{tabular}{lccccc} 
& \multicolumn{5}{c}{ Occ. MO } \\
\cline { 2 - 6 } & $\mathbf{2 9}$ & $\mathbf{3 0}$ & $\mathbf{3 1}$ & $\mathbf{3 2}$ & $\mathbf{3 3}$ \\
\hline $\mathbf{C 1}$ & 5.46 & 8.46 & 2.89 & 25.80 & 2.82 \\
$\mathbf{C 2}$ & 3.38 & 4.41 & 12.85 & 26.50 & 13.35 \\
$\mathbf{C 3}$ & 2.71 & 0.37 & 6.09 & 0.07 & 8.15 \\
$\mathbf{C 4}$ & 6.96 & 5.19 & 12.86 & 22.93 & 17.05 \\
C5 & 12.98 & 11.02 & 3.20 & 24.22 & 3.12 \\
C6 & 11.06 & 5.10 & 0.91 & 0.12 & 22.47 \\
H7 & 2.60 & 0.00 & 0.93 & 0.01 & 0.00 \\
H8 & 2.61 & 0.00 & 1.38 & 0.01 & 0.00 \\
H9 & 4.75 & 0.00 & 1.36 & 0.01 & 0.00 \\
H10 & 1.74 & 0.00 & 0.87 & 0.01 & 0.00 \\
O11 & 11.01 & 23.92 & 56.56 & 0.03 & 22.07 \\
O12 & 24.63 & 33.28 & 0.03 & 0.09 & 9.27 \\
C13 & 5.38 & 1.93 & 0.05 & 0.15 & 0.17 \\
H14 & 1.34 & 3.16 & 0.01 & 0.03 & 0.77 \\
H15 & 1.34 & 3.15 & 0.01 & 0.03 & 0.77 \\
H16 & 2.06 & 0.00 & 0.01 & 0.00 & 0.00
\end{tabular}

Compound 45:

Occ. MO

\begin{tabular}{lccccc} 
& $\mathbf{2 5}$ & $\mathbf{2 6}$ & $\mathbf{2 7}$ & $\mathbf{2 8}$ & $\mathbf{2 9}$ \\
\hline $\mathbf{C 1}$ & 12.05 & 13.80 & 2.98 & 23.61 & 2.39 \\
$\mathbf{C 2}$ & 6.37 & 5.25 & 12.20 & 25.49 & 17.16 \\
$\mathbf{C 3}$ & 3.71 & 0.99 & 6.58 & 0.07 & 7.95 \\
C4 & 6.20 & 5.46 & 12.82 & 26.53 & 15.34 \\
C5 & 12.18 & 13.58 & 3.20 & 24.07 & 2.07 \\
C6 & 15.46 & 15.95 & 1.12 & 0.08 & 27.28 \\
H7 & 3.19 & 0.00 & 1.13 & 0.01 & 0.00 \\
H8 & 5.53 & 0.00 & 1.56 & 0.01 & 0.00 \\
H9 & 5.20 & 0.00 & 1.52 & 0.01 & 0.00 \\
H10 & 3.74 & 0.00 & 1.19 & 0.01 & 0.00 \\
O11 & 16.52 & 34.53 & 55.71 & 0.00 & 23.05 \\
C12 & 4.31 & 4.30 & 0.00 & 0.05 & 0.72
\end{tabular}




$\begin{array}{llllll}\text { H13 } & 0.93 & 3.07 & 0.00 & 0.03 & 2.02 \\ \text { H14 } & 0.93 & 3.07 & 0.00 & 0.03 & 2.01 \\ \text { H15 } & 3.68 & 0.00 & 0.00 & 0.00 & 0.00\end{array}$

Compound 46:

Occ. MO

\begin{tabular}{lccccc} 
& $\mathbf{2 5}$ & $\mathbf{2 6}$ & $\mathbf{2 7}$ & $\mathbf{2 8}$ & $\mathbf{2 9}$ \\
\hline $\mathbf{C 1}$ & 10.46 & 17.10 & 3.16 & 20.04 & 1.23 \\
$\mathbf{C 2}$ & 5.15 & 6.44 & 12.71 & 23.46 & 17.62 \\
$\mathbf{C 3}$ & 3.45 & 1.16 & 6.90 & 0.09 & 7.36 \\
$\mathbf{C 4}$ & 7.02 & 3.27 & 12.65 & 28.60 & 17.39 \\
$\mathbf{C 5}$ & 16.37 & 10.41 & 3.11 & 22.31 & 1.66 \\
C6 & 14.13 & 21.12 & 1.08 & 0.16 & 29.84 \\
H7 & 4.29 & 0.00 & 1.09 & 0.01 & 0.00 \\
H8 & 3.79 & 0.00 & 1.55 & 0.01 & 0.00 \\
H9 & 5.93 & 0.00 & 1.57 & 0.01 & 0.00 \\
H10 & 0.17 & 0.01 & 0.00 & 0.00 & 0.01 \\
C11 & 8.23 & 3.00 & 1.58 & 1.82 & 0.13 \\
H12 & 1.60 & 1.98 & 0.02 & 1.67 & 0.40 \\
H13 & 1.60 & 1.98 & 0.02 & 1.68 & 0.40 \\
H14 & 0.79 & 0.00 & 0.19 & 0.00 & 0.00 \\
O15 & 17.04 & 33.52 & 54.36 & 0.14 & 23.95
\end{tabular}

Compound 47:

\begin{tabular}{lccccc} 
& \multicolumn{5}{c}{ Occ. MO } \\
\cline { 2 - 6 } & $\mathbf{2 1}$ & $\mathbf{2 2}$ & $\mathbf{2 3}$ & $\mathbf{2 4}$ & $\mathbf{2 5}$ \\
\hline C1 & 12.00 & 14.41 & 3.07 & 23.57 & 1.58 \\
C2 & 13.49 & 21.61 & 1.00 & 0.07 & 28.62 \\
C3 & 12.00 & 14.41 & 3.07 & 23.57 & 1.59 \\
C4 & 7.73 & 4.18 & 12.46 & 26.34 & 17.75 \\
C5 & 5.32 & 1.91 & 6.61 & 0.07 & 7.90 \\
C6 & 7.73 & 4.18 & 12.46 & 26.34 & 17.75 \\
H7 & 4.09 & 0.00 & 1.13 & 0.01 & 0.00 \\
H8 & 0.00 & 0.01 & 0.00 & 0.00 & 0.01 \\
H9 & 4.09 & 0.00 & 1.13 & 0.01 & 0.00 \\
H10 & 6.24 & 0.00 & 1.55 & 0.01 & 0.00 \\
H11 & 6.24 & 0.00 & 1.55 & 0.01 & 0.00 \\
O12 & 21.06 & 39.29 & 55.98 & 0.00 & 24.80
\end{tabular}

Compound 48:

Occ. MO

\begin{tabular}{lccccc} 
& $\mathbf{2 5}$ & $\mathbf{2 6}$ & $\mathbf{2 7}$ & $\mathbf{2 8}$ & $\mathbf{2 9}$ \\
\hline $\mathbf{C 1}$ & 9.48 & 11.91 & 2.80 & 25.57 & 3.14 \\
$\mathbf{C 2}$ & 7.05 & 5.22 & 12.68 & 25.13 & 14.69 \\
$\mathbf{C 3}$ & 4.75 & 0.63 & 6.04 & 0.07 & 8.56 \\
C4 & 9.08 & 5.46 & 12.95 & 24.25 & 15.67 \\
C5 & 12.51 & 12.65 & 3.06 & 24.86 & 3.42
\end{tabular}




$\begin{array}{lccccc}\mathbf{C 6} & 13.68 & 8.45 & 0.92 & 0.09 & 22.48 \\ \mathbf{H 7} & 3.62 & 0.00 & 0.92 & 0.01 & 0.00 \\ \mathbf{H} 8 & 5.35 & 0.00 & 1.40 & 0.01 & 0.00 \\ \mathbf{H 9} & 6.51 & 0.00 & 1.32 & 0.01 & 0.00 \\ \mathbf{H 1 0} & 2.37 & 0.00 & 0.88 & 0.01 & 0.00 \\ \mathbf{0 1 1} & 15.72 & 30.09 & 57.02 & 0.00 & 22.42 \\ \mathbf{0 1 2} & 8.82 & 25.57 & 0.00 & 0.00 & 9.61 \\ \mathbf{H 1 3} & 1.05 & 0.01 & 0.00 & 0.00 & 0.00\end{array}$

Compound 49:

Occ. MO

\begin{tabular}{lccccc} 
& $\mathbf{2 5}$ & $\mathbf{2 6}$ & $\mathbf{2 7}$ & $\mathbf{2 8}$ & $\mathbf{2 9}$ \\
\hline C1 & 10.64 & 13.67 & 2.85 & 25.26 & 2.00 \\
C2 & 9.33 & 3.49 & 12.78 & 24.64 & 17.24 \\
C3 & 5.59 & 2.06 & 5.90 & 0.07 & 7.70 \\
C4 & 9.33 & 3.49 & 12.78 & 24.64 & 17.23 \\
C5 & 10.64 & 13.67 & 2.85 & 25.26 & 2.00 \\
C6 & 12.42 & 17.03 & 0.79 & 0.10 & 24.47 \\
H7 & 2.77 & 0.00 & 0.81 & 0.01 & 0.00 \\
H8 & 6.72 & 0.00 & 1.30 & 0.01 & 0.00 \\
H9 & 6.72 & 0.00 & 1.30 & 0.01 & 0.00 \\
H10 & 2.77 & 0.00 & 0.81 & 0.01 & 0.00 \\
O11 & 16.12 & 36.34 & 57.83 & 0.00 & 24.33 \\
F12 & 6.94 & 10.24 & 0.00 & 0.00 & 5.01
\end{tabular}

Compound 50:

Occ. MO

\begin{tabular}{lccccc} 
& $\mathbf{2 5}$ & $\mathbf{2 6}$ & $\mathbf{2 7}$ & $\mathbf{2 8}$ & $\mathbf{2 9}$ \\
\hline $\mathbf{C 1}$ & 16.14 & 1.87 & 2.56 & 20.78 & 1.30 \\
$\mathbf{C 2}$ & 5.63 & 2.23 & 11.54 & 24.25 & 17.55 \\
$\mathbf{C 3}$ & 1.39 & 2.04 & 6.90 & 0.08 & 7.43 \\
$\mathbf{C 4}$ & 3.66 & 7.50 & 10.91 & 27.93 & 17.72 \\
C5 & 12.07 & 4.56 & 3.34 & 22.61 & 1.78 \\
C6 & 21.28 & 4.13 & 0.58 & 0.13 & 29.58 \\
H7 & 0.00 & 0.95 & 0.70 & 0.01 & 0.00 \\
H8 & 0.00 & 0.24 & 1.31 & 0.01 & 0.00 \\
H9 & 0.00 & 0.75 & 1.15 & 0.01 & 0.00 \\
H10 & 0.01 & 0.74 & 0.03 & 0.00 & 0.01 \\
O11 & 35.36 & 5.46 & 52.25 & 0.08 & 24.12 \\
N12 & 2.51 & 67.29 & 8.67 & 2.29 & 0.04 \\
H13 & 0.98 & 1.12 & 0.03 & 0.91 & 0.23 \\
H14 & 0.98 & 1.12 & 0.03 & 0.91 & 0.23
\end{tabular}

Compound 51:

\begin{tabular}{lccccc} 
& \multicolumn{5}{c}{ Occ. MO } \\
\cline { 2 - 6 } & $\mathbf{2 9}$ & $\mathbf{3 0}$ & $\mathbf{3 1}$ & $\mathbf{3 2}$ & $\mathbf{3 3}$ \\
\hline $\mathbf{C 1}$ & 6.86 & 17.60 & 3.27 & 16.03 & 0.68
\end{tabular}




$\begin{array}{lccccc}\mathbf{C 2} & 2.86 & 7.89 & 12.64 & 18.86 & 18.86 \\ \mathbf{C 3} & 1.89 & 0.57 & 6.08 & 0.17 & 6.76 \\ \mathbf{C 4} & 4.62 & 1.28 & 12.09 & 30.39 & 15.88 \\ \mathbf{C 5} & 13.08 & 3.01 & 2.84 & 16.06 & 1.99 \\ \mathbf{C 6} & 13.72 & 18.12 & 0.64 & 0.34 & 30.95 \\ \mathbf{H 7} & 3.65 & 0.00 & 0.84 & 0.01 & 0.00 \\ \mathbf{H} 8 & 0.69 & 0.00 & 1.23 & 0.01 & 0.01 \\ \mathbf{H 9} & 4.39 & 0.00 & 1.11 & 0.01 & 0.00 \\ \mathbf{H 1 0} & 1.51 & 0.01 & 0.01 & 0.00 & 0.01 \\ \mathbf{O 1 1} & 7.01 & 23.22 & 58.13 & 0.73 & 23.84 \\ \mathbf{O 1 2} & 28.11 & 23.00 & 0.92 & 15.22 & 0.53 \\ \mathbf{C 1 3} & 6.00 & 1.18 & 0.15 & 0.18 & 0.22 \\ \mathbf{H} 14 & 1.47 & 2.05 & 0.00 & 0.99 & 0.14 \\ \mathbf{H 1 5} & 1.51 & 2.05 & 0.00 & 0.99 & 0.14 \\ \mathbf{H 1 6} & 2.64 & 0.00 & 0.05 & 0.00 & 0.00\end{array}$

Compound 52:

Occ. MO

\begin{tabular}{lccccc} 
& $\mathbf{2 5}$ & $\mathbf{2 6}$ & $\mathbf{2 7}$ & $\mathbf{2 8}$ & $\mathbf{2 9}$ \\
\hline C1 & 8.30 & 18.56 & 3.13 & 16.15 & 0.61 \\
C2 & 7.00 & 7.95 & 12.65 & 17.70 & 21.64 \\
C3 & 5.48 & 0.70 & 6.41 & 0.20 & 6.64 \\
C4 & 10.85 & 2.40 & 12.00 & 35.26 & 12.79 \\
C5 & 17.38 & 4.90 & 2.63 & 16.15 & 2.37 \\
C6 & 12.97 & 19.85 & 0.68 & 0.06 & 31.94 \\
H7 & 5.70 & 0.01 & 0.85 & 0.01 & 0.00 \\
H8 & 2.37 & 0.00 & 1.33 & 0.01 & 0.01 \\
H9 & 7.56 & 0.00 & 1.21 & 0.01 & 0.00 \\
H10 & 2.78 & 0.01 & 0.01 & 0.00 & 0.01 \\
O11 & 13.51 & 28.13 & 57.64 & 1.15 & 22.66 \\
O12 & 4.94 & 17.49 & 1.46 & 13.30 & 1.34 \\
H13 & 1.14 & 0.01 & 0.01 & 0.01 & 0.00
\end{tabular}

Compound 53:

Occ. MO

\begin{tabular}{lccccc} 
& $\mathbf{2 9}$ & $\mathbf{3 0}$ & $\mathbf{3 1}$ & $\mathbf{3 2}$ & $\mathbf{3 3}$ \\
\hline $\mathbf{C 1}$ & 3.88 & 7.47 & 2.78 & 24.76 & 1.16 \\
C2 & 1.27 & 3.56 & 12.69 & 25.14 & 16.08 \\
C3 & 0.20 & 0.44 & 6.07 & 0.07 & 6.51 \\
C4 & 1.27 & 3.56 & 12.69 & 25.14 & 16.08 \\
C5 & 3.88 & 7.47 & 2.78 & 24.77 & 1.16 \\
C6 & 2.79 & 6.60 & 0.86 & 0.09 & 27.59 \\
H7 & 0.14 & 0.00 & 0.82 & 0.01 & 0.00 \\
H8 & 0.72 & 0.00 & 1.29 & 0.01 & 0.00 \\
H9 & 0.72 & 0.00 & 1.29 & 0.01 & 0.00 \\
H10 & 0.14 & 0.00 & 0.82 & 0.01 & 0.00 \\
O11 & 0.67 & 19.16 & 57.86 & 0.00 & 22.22
\end{tabular}




$\begin{array}{llllll}\text { Cl12 } & 84.30 & 51.73 & 0.04 & 0.00 & 9.18\end{array}$

Compound 54:

Occ. MO

\begin{tabular}{lccccc} 
& $\mathbf{3 8}$ & $\mathbf{3 9}$ & $\mathbf{4 0}$ & $\mathbf{4 1}$ & $\mathbf{4 2}$ \\
\hline C1 & 2.14 & 3.80 & 2.73 & 24.68 & 1.13 \\
C2 & 0.73 & 3.08 & 12.49 & 25.22 & 15.95 \\
C3 & 0.09 & 0.07 & 6.12 & 0.07 & 6.14 \\
C4 & 0.73 & 3.08 & 12.50 & 25.22 & 15.94 \\
C5 & 2.14 & 3.80 & 2.73 & 24.68 & 1.13 \\
C6 & 1.13 & 1.40 & 0.78 & 0.09 & 26.88 \\
H7 & 0.06 & 0.00 & 0.82 & 0.01 & 0.00 \\
H8 & 0.27 & 0.00 & 1.24 & 0.01 & 0.00 \\
H9 & 0.27 & 0.00 & 1.24 & 0.01 & 0.00 \\
H10 & 0.06 & 0.00 & 0.82 & 0.01 & 0.00 \\
O11 & 0.06 & 10.56 & 57.90 & 0.00 & 21.63 \\
Br12 & 92.31 & 74.20 & 0.63 & 0.00 & 11.20
\end{tabular}

Compound 55:

Occ. MO

\begin{tabular}{lccccc} 
& $\mathbf{2 5}$ & $\mathbf{2 6}$ & $\mathbf{2 7}$ & $\mathbf{2 8}$ & $\mathbf{2 9}$ \\
\hline $\mathbf{C 1}$ & 10.62 & 14.03 & 3.19 & 21.47 & 0.72 \\
C2 & 8.76 & 4.21 & 12.72 & 23.09 & 19.24 \\
C3 & 5.96 & 1.88 & 5.95 & 0.08 & 6.77 \\
C4 & 9.32 & 3.69 & 11.97 & 29.37 & 16.42 \\
C5 & 13.75 & 11.43 & 2.53 & 19.57 & 1.84 \\
C6 & 12.90 & 21.56 & 0.55 & 0.07 & 30.37 \\
H7 & 5.84 & 0.00 & 0.76 & 0.01 & 0.00 \\
H8 & 3.84 & 0.00 & 1.19 & 0.01 & 0.01 \\
H9 & 6.99 & 0.00 & 0.98 & 0.01 & 0.00 \\
H10 & 1.20 & 0.01 & 0.01 & 0.00 & 0.01 \\
O11 & 13.87 & 35.69 & 59.26 & 0.12 & 24.19 \\
F12 & 6.95 & 7.49 & 0.89 & 6.21 & 0.43
\end{tabular}

\section{Compound 56:}

Occ. MO

\begin{tabular}{lccccc} 
& $\mathbf{3 8}$ & $\mathbf{3 9}$ & $\mathbf{4 0}$ & $\mathbf{4 1}$ & $\mathbf{4 2}$ \\
\hline C1 & 10.72 & 1.36 & 2.75 & 17.10 & 0.88 \\
C2 & 6.21 & 0.49 & 11.23 & 17.85 & 18.70 \\
C3 & 0.08 & 0.36 & 6.11 & 0.06 & 6.72 \\
C4 & 0.09 & 2.10 & 12.38 & 23.08 & 17.23 \\
C5 & 0.23 & 1.12 & 3.25 & 18.32 & 1.75 \\
C6 & 6.49 & 2.73 & 0.74 & 0.09 & 29.55 \\
H7 & 0.00 & 0.39 & 0.80 & 0.01 & 0.00 \\
H8 & 0.00 & 0.14 & 1.24 & 0.01 & 0.00 \\
H9 & 0.00 & 0.11 & 1.07 & 0.01 & 0.00 \\
H10 & 0.00 & 0.03 & 0.00 & 0.00 & 0.01
\end{tabular}




$\begin{array}{lccccc}011 & 7.95 & 1.65 & 51.40 & 0.09 & 24.30 \\ \text { Br12 } & 68.21 & 89.51 & 9.01 & 23.40 & 0.86\end{array}$

Compound 57:

Occ. MO

\begin{tabular}{lccccc} 
& $\mathbf{2 9}$ & $\mathbf{3 0}$ & $\mathbf{3 1}$ & $\mathbf{3 2}$ & $\mathbf{3 3}$ \\
\hline $\mathbf{C 1}$ & 2.17 & 12.80 & 2.86 & 19.29 & 0.87 \\
C2 & 0.40 & 5.46 & 12.08 & 20.48 & 17.97 \\
C3 & 0.54 & 0.58 & 5.86 & 0.07 & 6.86 \\
C4 & 2.39 & 1.36 & 13.07 & 24.06 & 17.89 \\
C5 & 2.52 & 4.18 & 3.11 & 20.47 & 1.92 \\
C6 & 4.59 & 13.14 & 0.70 & 0.08 & 29.18 \\
H7 & 0.77 & 0.00 & 0.83 & 0.01 & 0.00 \\
H8 & 0.20 & 0.00 & 1.26 & 0.01 & 0.00 \\
H9 & 0.17 & 0.00 & 0.96 & 0.01 & 0.00 \\
H10 & 0.01 & 0.00 & 0.00 & 0.00 & 0.01 \\
O11 & 0.28 & 18.98 & 55.75 & 0.08 & 24.57 \\
Cl12 & 85.96 & 43.50 & 3.50 & 15.45 & 0.73
\end{tabular}

Compound 58:

Occ. MO

\begin{tabular}{lccccc} 
& $\mathbf{2 7}$ & $\mathbf{2 8}$ & $\mathbf{2 9}$ & $\mathbf{3 0}$ & $\mathbf{3 1}$ \\
\hline $\mathbf{C 1}$ & 2.88 & 14.58 & 3.16 & 22.76 & 1.02 \\
C2 & 1.23 & 5.19 & 12.47 & 21.47 & 17.03 \\
$\mathbf{C 3}$ & 1.03 & 1.09 & 5.54 & 0.06 & 7.29 \\
$\mathbf{C 4}$ & 3.06 & 1.90 & 13.28 & 21.53 & 19.43 \\
C5 & 10.24 & 8.46 & 2.99 & 23.50 & 1.83 \\
C6 & 5.01 & 16.56 & 0.79 & 0.30 & 26.84 \\
H7 & 1.68 & 0.00 & 0.79 & 0.01 & 0.00 \\
H8 & 0.28 & 0.00 & 1.15 & 0.01 & 0.00 \\
H9 & 1.18 & 0.00 & 0.95 & 0.01 & 0.00 \\
H10 & 0.48 & 0.01 & 0.00 & 0.00 & 0.01 \\
O11 & 1.22 & 26.36 & 57.70 & 0.00 & 25.93 \\
C12 & 28.34 & 7.84 & 0.86 & 0.87 & 0.02 \\
N13 & 43.38 & 18.00 & 0.33 & 9.49 & 0.60
\end{tabular}

Compound 59:

Occ. MO

\begin{tabular}{lccccc} 
& $\mathbf{2 5}$ & $\mathbf{2 6}$ & $\mathbf{2 7}$ & $\mathbf{2 8}$ & $\mathbf{2 9}$ \\
\hline C1 & 8.32 & 1.25 & 3.17 & 27.05 & 1.16 \\
C2 & 1.61 & 0.25 & 11.99 & 24.36 & 16.46 \\
C3 & 2.41 & 0.43 & 5.22 & 0.07 & 7.25 \\
C4 & 2.35 & 0.81 & 14.06 & 18.00 & 20.73 \\
C5 & 15.46 & 2.63 & 3.78 & 23.91 & 1.72 \\
C6 & 15.25 & 1.81 & 1.04 & 0.89 & 25.66 \\
H7 & 0.00 & 0.00 & 0.72 & 0.01 & 0.00 \\
H8 & 0.00 & 0.00 & 1.01 & 0.01 & 0.00
\end{tabular}




$\begin{array}{lccccc}\text { H9 } & 0.00 & 0.00 & 0.75 & 0.01 & 0.01 \\ \text { H10 } & 0.00 & 0.00 & 0.02 & 0.00 & 0.01 \\ \text { O11 } & 29.69 & 5.25 & 56.03 & 0.25 & 26.22 \\ \text { N12 } & 0.35 & 0.48 & 1.21 & 0.10 & 0.07 \\ \text { H13 } & 20.94 & 35.99 & 0.22 & 3.38 & 0.04 \\ \text { H14 } & 3.62 & 51.10 & 0.76 & 1.96 & 0.67\end{array}$

Compound 60:

\begin{tabular}{lccccc} 
& \multicolumn{5}{c}{ Occ. MO } \\
\cline { 2 - 6 } & $\mathbf{2 7}$ & $\mathbf{2 8}$ & $\mathbf{2 9}$ & $\mathbf{3 0}$ & $\mathbf{3 1}$ \\
\hline $\mathbf{C 1}$ & 3.99 & 9.36 & 3.22 & 24.03 & 0.28 \\
C2 & 1.40 & 5.08 & 13.05 & 25.89 & 16.48 \\
C3 & 0.44 & 0.71 & 6.85 & 0.07 & 4.43 \\
C4 & 1.43 & 4.97 & 12.69 & 26.00 & 16.60 \\
C5 & 4.03 & 9.21 & 3.09 & 23.90 & 0.33 \\
C6 & 8.76 & 9.94 & 1.30 & 0.07 & 31.25 \\
H7 & 0.55 & 0.00 & 1.06 & 0.01 & 0.00 \\
H8 & 1.04 & 0.00 & 1.42 & 0.01 & 0.00 \\
H9 & 1.06 & 0.00 & 1.44 & 0.01 & 0.00 \\
H10 & 0.54 & 0.00 & 1.02 & 0.01 & 0.00 \\
O11 & 3.18 & 24.13 & 54.82 & 0.00 & 20.40 \\
C12 & 30.25 & 9.87 & 0.01 & 0.00 & 0.14 \\
N13 & 43.32 & 26.72 & 0.04 & 0.00 & 10.08
\end{tabular}

Compound 61:

Occ. MO

\begin{tabular}{lccccc} 
& $\mathbf{2 5}$ & $\mathbf{2 6}$ & $\mathbf{2 7}$ & $\mathbf{2 8}$ & $\mathbf{2 9}$ \\
\hline C1 & 11.42 & 0.12 & 3.16 & 23.63 & 0.13 \\
C2 & 3.69 & 0.02 & 12.79 & 26.03 & 16.14 \\
C3 & 2.68 & 0.00 & 6.86 & 0.07 & 3.23 \\
C4 & 3.66 & 0.02 & 12.77 & 25.96 & 16.26 \\
C5 & 11.38 & 0.12 & 3.16 & 23.65 & 0.13 \\
C6 & 23.76 & 0.00 & 0.83 & 0.08 & 30.50 \\
H7 & 0.00 & 0.00 & 0.93 & 0.01 & 0.00 \\
H8 & 0.00 & 0.00 & 1.28 & 0.01 & 0.00 \\
H9 & 0.00 & 0.00 & 1.28 & 0.01 & 0.00 \\
H10 & 0.00 & 0.00 & 0.93 & 0.01 & 0.00 \\
O11 & 33.46 & 0.00 & 55.14 & 0.00 & 19.43 \\
N12 & 0.35 & 0.44 & 0.02 & 0.00 & 1.78 \\
H13 & 4.80 & 49.63 & 0.42 & 0.28 & 6.19 \\
H14 & 4.79 & 49.65 & 0.42 & 0.27 & 6.19
\end{tabular}


Part S5 - Complete correlation results for Carboxylate ions:

S5A) Linear regression parameters for $p K_{a}$ values versus $\mathrm{HOMO}$ energies ${ }^{a}$ plots for carboxylate ions.

\begin{tabular}{lcccccc}
\hline Methodology & $\mathbf{r}^{2}$ & $\mathbf{a}^{b}$ & $\mathbf{b}^{c}$ & $\begin{array}{c}\mathbf{p K}_{\mathbf{a}} \\
\mathbf{e r r o r}\end{array}$ & $\mathbf{S D}^{d}$ & $\boldsymbol{F}$ \\
\hline HF/PCM/6-31G(d,p) & 0.444 & $28.0(13.5)$ & $13.5(4.9)$ & 2.40 & 0.945 & 27.1 \\
HF/PCM/6-31+G(d,p) & 0.385 & $22.6(13.2)$ & $12.0(5.1)$ & 2.71 & 0.994 & 21.3 \\
DFT/PCM/6-31G(d,p) & 0.461 & $83.6(22.7)$ & $22.0(5.1)$ & 1.41 & 0.930 & 29.1 \\
DFT/PCM/6-31+G(d,p) & 0.662 & $102.8(18.2)$ & $29.8(4.7)$ & 1.11 & 0.737 & 66.5 \\
\hline
\end{tabular}

S5B) Linear regression parameters for $p K_{\mathrm{a}}$ values versus $p_{z}$-type $\mathrm{MO}$ energies ${ }^{a}$ plots for carboxylate ions.

\begin{tabular}{lcccccc}
\hline Methodology & $\mathbf{r}^{\mathbf{2}}$ & $\mathbf{a}^{\boldsymbol{b}}$ & $\mathbf{b}^{\boldsymbol{c}}$ & $\begin{array}{c}\mathbf{p} \boldsymbol{K}_{\mathbf{a}} \\
\text { error }\end{array}$ & $\mathbf{S D}^{\boldsymbol{d}}$ & $\boldsymbol{F}$ \\
\hline HF/PCM/6-31G(d,p) & 0.718 & $139.5(19.0)$ & $59.4(7.6)$ & 0.90 & 0.673 & 86.5 \\
HF/PCM/6-31+G(d,p) & 0.787 & $171.9(17.9)$ & $76.6(7.6)$ & 0.72 & 0.585 & 125.4 \\
DFT/PCM/6-31G(d,p) & 0.729 & $133.9(18.2)$ & $35.9(4.4)$ & 0.90 & 0.660 & 91.4 \\
DFT/PCM/6-31+G(d,p) & 0.802 & $178.1(17.5)$ & $53.8(5.0)$ & 0.69 & 0.564 & 137.8 \\
\hline
\end{tabular}

S5C) Linear regression parameters for $p K_{\mathrm{a}}$ values versus $p_{x} p_{y}$-type $\mathrm{MO}$ energies ${ }^{a}$ plots for carboxylate ions.

\begin{tabular}{lcccccc}
\hline Methodology & $\mathbf{r}^{2}$ & $\mathbf{a}^{b}$ & $\mathbf{b}^{c}$ & $\begin{array}{c}\mathbf{p} \boldsymbol{K}_{\mathbf{a}} \\
\mathbf{e r r o r}\end{array}$ & $\mathbf{S D}^{d}$ & $\boldsymbol{F}$ \\
\hline HF/PCM/6-31G(d,p) & 0.891 & $128.1(10.7)$ & $56.6(4.5)$ & 0.61 & 0.418 & 278.3 \\
HF/PCM/6-31+G(d,p) & 0.788 & $129.7(15.5)$ & $60.3(6.8)$ & 0.82 & 0.583 & 126.7 \\
DFT/PCM/6-31G(d,p) & 0.809 & $125.4(14.4)$ & $32.0(3.3)$ & 0.80 & 0.554 & 143.8 \\
DFT/PCM/6-31+G(d,p) & 0.917 & $145.1(9.9)$ & $42.0(2.6)$ & 0.51 & 0.366 & 373.5 \\
\hline
\end{tabular}


Part S6 - Complete correlation results for Alcohols and Phenols anions:

S6A) Linear regression parameters for $p K_{a}$ values versus $\mathrm{HOMO}$ energies ${ }^{a}$ plots for alkoxide and phenoxide ions.

\begin{tabular}{ccccccc}
\hline Methodology & $\mathbf{r}^{\mathbf{2}}$ & $\mathbf{a}^{\boldsymbol{b}}$ & $\mathbf{b}^{\boldsymbol{c}}$ & $\begin{array}{c}\mathbf{p} \boldsymbol{K}_{\mathbf{a}} \\
\text { error }\end{array}$ & $\mathbf{S D}^{d}$ & $\boldsymbol{F}$ \\
\hline HF/PCM/6-31G(d,p) & 0.644 & $-67.0(24.3)$ & $-7.30(6.66)$ & 4.44 & 1.86 & 41.6 \\
HF/PCM/6-31+G(d,p) & 0.683 & $-62.3(21.8)$ & $-7.20(6.40)$ & 4.40 & 1.75 & 49.6 \\
DFT/PCM/6-31G(d,p) & $9.0 \times 10^{-5}$ & $-2.72(75.5)$ & $10.5(12.6)$ & 4.22 & 3.11 & 0.002 \\
DFT/PCM/6-31+G(d,p) & 0.381 & $-113.2(52.1)$ & $-11.4(10.3)$ & 4.08 & 2.57 & 10.7 \\
\hline
\end{tabular}

S6B) Linear regression parameters for $p K_{a}$ values versus $\mathrm{HOMO}$ energies ${ }^{a}$ plots for phenoxide ions.

\begin{tabular}{ccccccc}
\hline Methodology & $\mathbf{r}^{2}$ & $\mathbf{a}^{\boldsymbol{b}}$ & $\mathbf{b}^{\boldsymbol{c}}$ & $\begin{array}{c}\mathbf{p} \boldsymbol{K}_{\mathbf{a}} \\
\text { error }\end{array}$ & $\mathbf{S D}^{\boldsymbol{d}}$ & $\boldsymbol{F}$ \\
\hline HF/PCM/6-31G(d,p) & 0.877 & $78.0(7.8)$ & $29.1(2.0)$ & 0.38 & 0.304 & 121.3 \\
HF/PCM/6-31+G(d,p) & 0.855 & $81.3(8.7)$ & $31.3(2.3)$ & 0.40 & 0.330 & 100.0 \\
DFT/PCM/6-31G(d,p) & 0.922 & $75.8(6.0)$ & $21.8(1.0)$ & 0.31 & 0.242 & 200.1 \\
DFT/PCM/6-31+G(d,p) & 0.898 & $80.2(7.1)$ & $24.6(1.4)$ & 0.34 & 0.277 & 149.3 \\
\hline
\end{tabular}

S6C) Linear regression parameters for $p K_{\mathrm{a}}$ values versus $p_{x} p_{y}$-type $\mathrm{MO}$ energies ${ }^{a}$ plots for alkoxide and phenoxide ions.

\begin{tabular}{lcccccc}
\hline \multicolumn{1}{c}{ Methodology } & $\mathbf{r}^{2}$ & $\mathbf{a}^{b}$ & $\mathbf{b}^{c}$ & $\begin{array}{c}\mathbf{p} \boldsymbol{K}_{\mathbf{a}} \\
\mathbf{e r r o r}\end{array}$ & $\mathbf{S D}^{d}$ & $\boldsymbol{F}$ \\
\hline HF/PCM/6-31G(d,p) & 0.975 & $144.8(8.6)$ & $64.3(3.2)$ & 0.91 & 0.494 & 888.3 \\
HF/PCM/6-31+G(d,p) & 0.960 & $160.9(11.4)$ & $74.0(4.5)$ & 1.08 & 0.621 & 554.6 \\
DFT/PCM/6-31G(d,p) & 0.973 & $174.0(9.8)$ & $45.9(2.0)$ & 0.87 & 0.514 & 819.7 \\
DFT/PCM/6-31+G(d,p) & 0.933 & $241.4(18.2)$ & $68.5(4.4)$ & 1.16 & 0.806 & 320.2 \\
\hline
\end{tabular}


Part S7 - Complete References:

(72) Frisch, M. J.; Trucks, G. W.; Schlegel, H. B.; Scuseria, G. E.; Robb, M. A.; Cheeseman, J. R.; Zakrzewski, V. G.; Montgomery, J. A. Jr.; Stratmann, R. E.; Burant, J. C.; Dapprich, S.; Millam, J. M.; Daniels, A. D.; Kudin, K. N.; Strain, M. C.; Farkas, O.; Tomasi, J.; Barone, V.; Cossi, M.; Cammi, R.; Mennucci, B.; Pomelli, C.; Adamo, C.; Clifford, S.; Ochterski, J.; Petersson, G. A.; Ayala, R. Y.; Cui, Q.; Morokuma, K.; Salvador, P.; Dannenberg, J. J.; Malick, D. K.; Rabuck, A. D.; Raghavachari, K.; Foresman, J. B.; Cioslowski, J.; Ortiz, J. V.; Baboul, A. G.; Stefanov, B. B.; Liu, G.; Liashenko, A.; Piskorz, P.; Komaromi, I.; Gomperts, R.; Martin, R. L.; Fox, D. J.; Keith, T.; Al-Laham, M. A.; Peng, C. Y.; Nanayakkara, A.; Challacombe, M.; Gill, P. M. W.; Johnson, B.; Chen, W.; Wong, M. W.; Andres, J. L.; Gonzalez, C.; Head-Gordon, M.; Replogle, E. S.; Pople, J. A. Gaussian 98, Revision A.11, Gaussian, Inc.: Pittsburgh PA, 2001. 